\title{
Exploiting Wi-Fi Channel State Information for Artificial Intelligence-Based Human Activity Recognition of Similar Dynamic Motions
}

by

Itaf Omar Joudeh

A thesis submitted to the Faculty of Graduate and Postdoctoral Affairs in partial fulfillment of the requirements for the degree of

Master of Applied Science

in

Electrical and Computer Engineering (Data Science)

Carleton University

Ottawa, Ontario

(C) 2020, Itaf Omar Joudeh 


\section{Copyright}

Parts of this thesis come from [1], "WiFi Channel State Information-Based Recognition of Sitting-Down and Standing-Up Activities," a conference proceedings paper, published by the Institute of Electrical and Electronics Engineers (IEEE). The IEEE does not require individuals working on a thesis to obtain a formal reuse license, however, in the text, material from this source will be identified as (C) 2019 [1], accordingly. 


\begin{abstract}
Real-time recognition of Activities of Daily Living (ADLs) enables surveillance, security, and health monitoring applications. Establishing systems to recognize ADLs nonintrusively and passively, would facilitate smart spaces by providing assistive living for seniors and people with disabilities. This work applies artificial intelligence to pervasive Wi-Fi technology to measure motion and distinguish the patterns associated with dynamic activities. Processed signals are segmented into labelled sequences. Statistical features are extracted to enable activity recognition. Classifiers are tested to predict activities. Support vector machines achieve the best performance at distinguishing still, sitting-down, and standing-up activity classes. Results on data collected by one subject achieve a classification accuracy of $98.8 \%$ for sitting and standing activities at one room location, within one experimental setup, and $98.5 \%$ for various activity locations, within different experimental setups. Results on data collected by four subjects achieve an accuracy of $97.6 \%$ for sitting and standing activities at random room locations.
\end{abstract}




\section{Acknowledgements}

This study was partially funded by a Discovery Grant and an Engage Grant from the Natural Sciences and Engineering Research Council (NSERC) of Canada, and was performed in collaboration with Aerial Technologies, Inc.

I would like to thank all members of my family, one by one, for their continued, unconditional love and support. I love them more than anything in this whole world!

Also, I would like to thank my supervisors, Dr. Cretu, Dr. Wallace, and Dr. Goubran, for their time and assistance. I would like to acknowledge my medical advisor, Dr. Knoefel, for being responsive and available. It was a great pleasure to work with them!

Nonetheless, I would also like to acknowledge my colleagues in the DSP lab at Carleton University, for all their help in performing some experiments. I enjoyed working with such nice people!

Last but not least, I really appreciate the services provided by the technical staff in the Department of Systems and Computer Engineering (SCE) at Carleton University. 


\section{Table of Contents}

Copyright t................................................................................................................................ ii

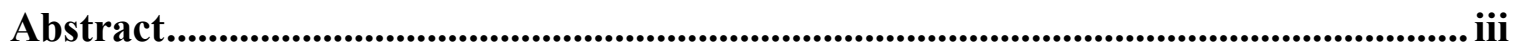

Acknowledgements ................................................................................................................... iv

Table of Contents ............................................................................................................................ v

List of Tables .............................................................................................................................. ix

List of Figures.................................................................................................................................. xiii

List of Appendices........................................................................................................................... xvi

Table of Acronyms....................................................................................................................... xvii

Chapter 1: Introduction ............................................................................................ 1

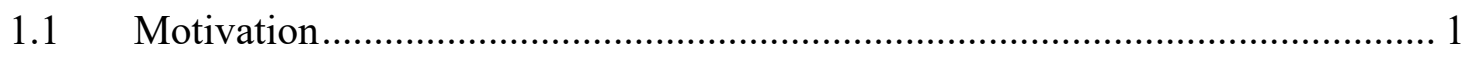

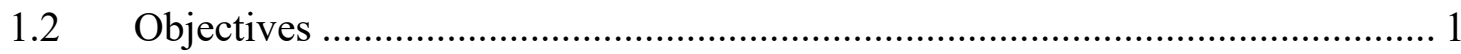

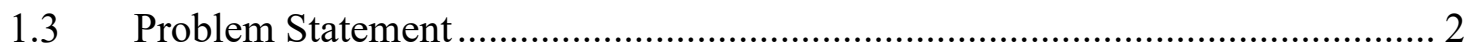

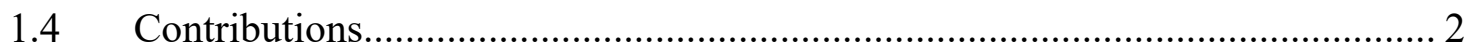

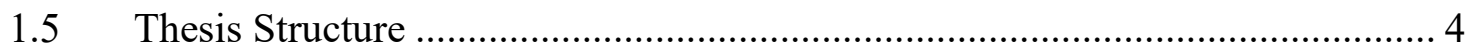

Chapter 2: Background .......................................................................................................... 5

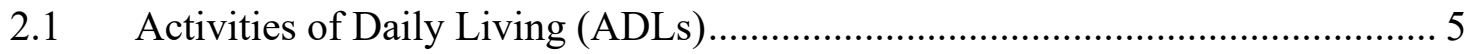

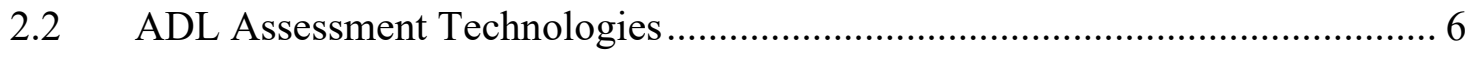

2.2.1 Usability of ADL Sensing Options for Aging Adults ........................................ 6

2.2.2 ADL Assessment with Cameras ................................................................... 7

2.2.3 ADL Assessment with Sensors and Smartphones........................................... 8

2.2.4 ADL Assessment with Radar ..................................................................... 10

2.2.5 ADL Assessment with Wi-Fi ................................................................... 10 


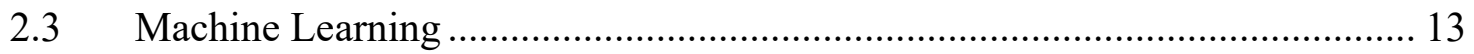

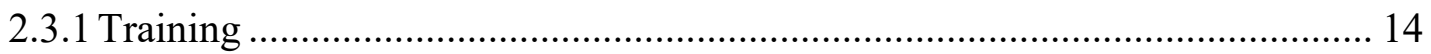

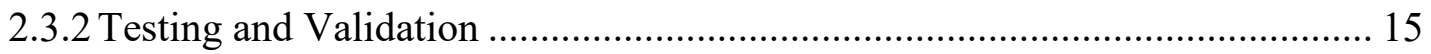

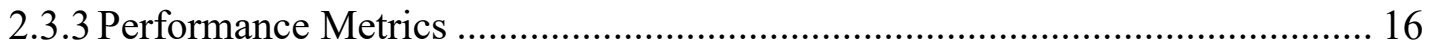

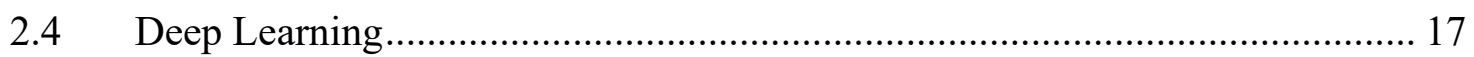

Chapter 3: Equipment............................................................................................................ 19

3.1 Theory of Operation of the Equipment ..................................................... 19

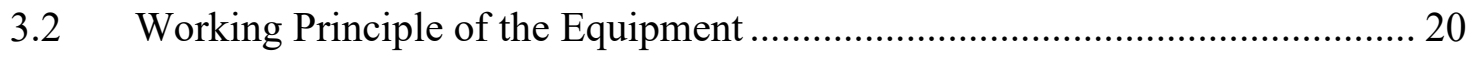

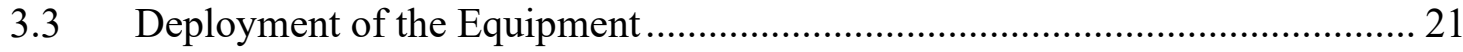

Chapter 4: Proposed Solution................................................................................................... 23

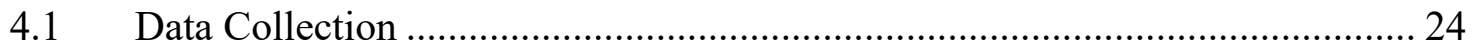

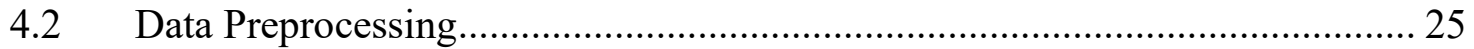

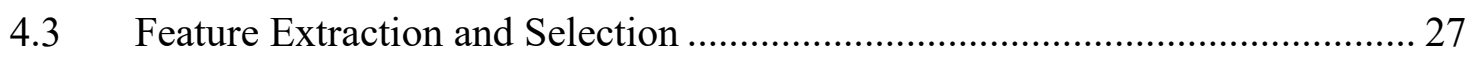

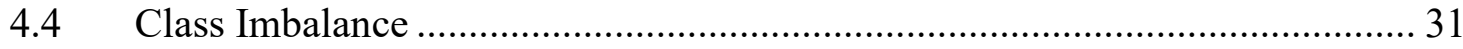

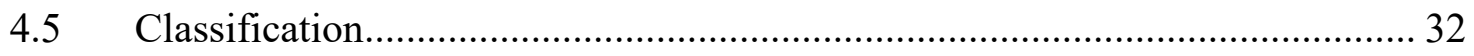

4.5.1 Classification via Traditional Machine Learning ............................................ 32

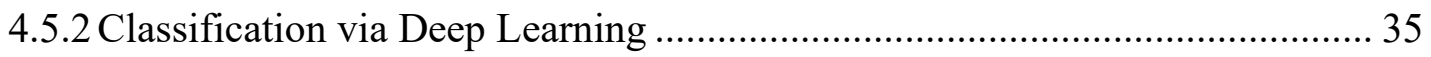

Chapter 5: Experimental Results ....................................................................................... 38

5.1 Distinguishing Sitting-down and Standing-up Activities ................................. 38

5.1.1 Preliminary Sitting and Standing Experiments by One Subject....................... 38

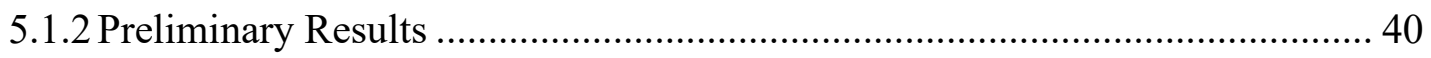

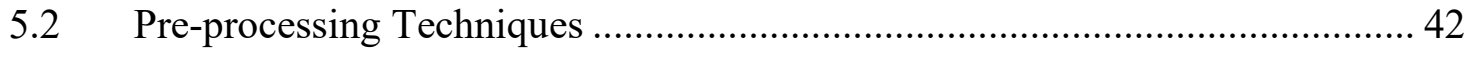

5.2.1 Experiments with Data Preprocessing Techniques .......................................... 43

5.2.2 CSI Normalization........................................................................................ 44 
5.2.3 Feature Scaling 44

5.2.4. Optimization of Preprocessing Methodology ............................................... 46

5.3 Subject Activity and Device Location Independence.................................. 48

5.3.1 Experiments on Activity Location Independence ..................................... 48

5.3.2 Independence of Activity Location .................................................... 52

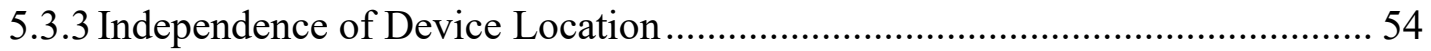

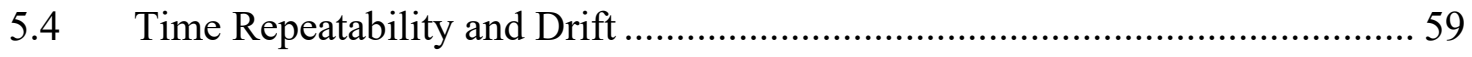

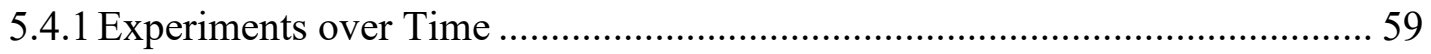

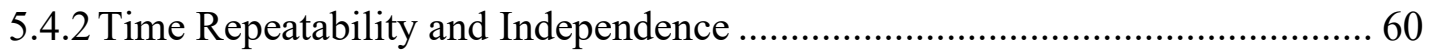

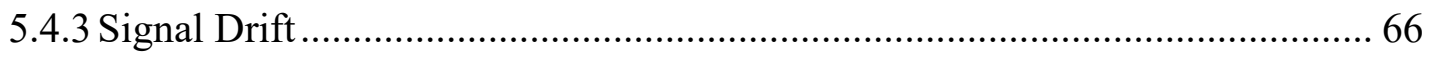

5.5 Testing and Validation on More Subjects............................................. 70

5.5.1 Experiments by Other Subjects ......................................................... 70

5.5.2 Dependence of Classification Performance on Subjects .............................. 71

5.5.3 Independence of Classification Performance from Subjects......................... 74

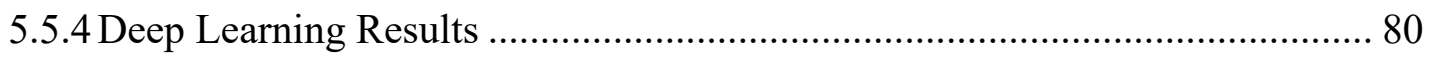

5.5.4.1 Sequence-to-Sequence BiLSTM Network...................................... 81

5.5.4.2 Sequence-to-Label BiLSTM Network ......................................... 81

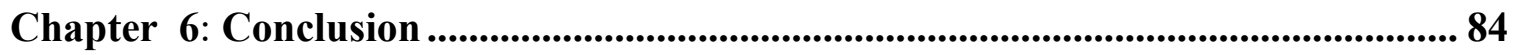

6.1 Summary of Related Work .................................................................... 84

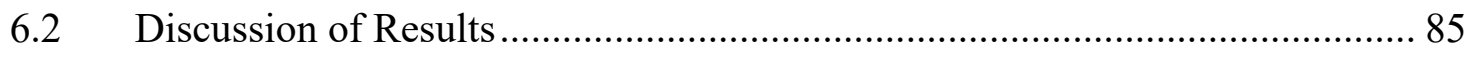

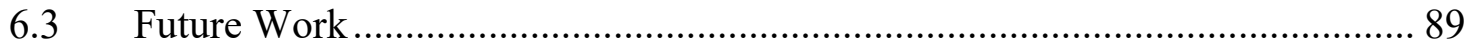

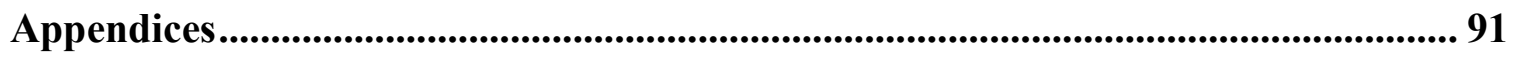

Appendix A Summary of Data Samples ........................................................ 91

Appendix B Samples of Preprocessed Data....................................................... 94 


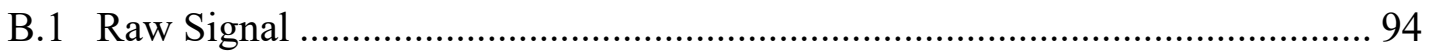

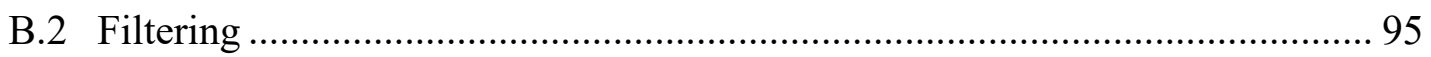

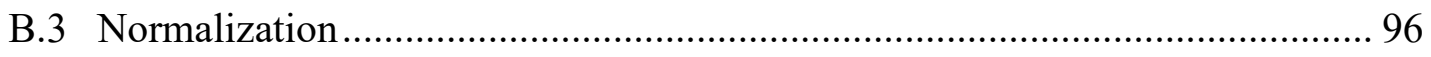

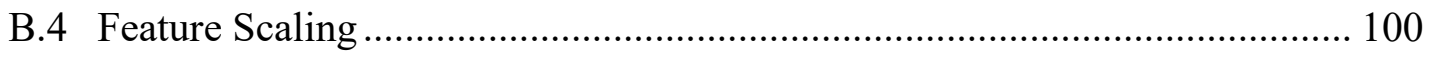

B.5 Filtering, Z-Score Standardization, and Feature Scaling Combined............. 103

Appendix C Samples of Data from Different Activity Locations ........................... 104

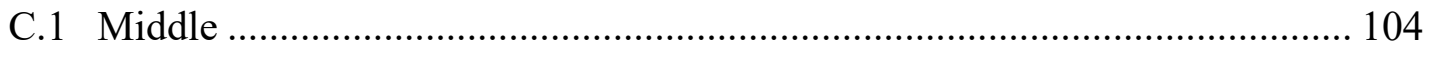

C.2 Near the Transmitter.................................................................................. 105

C.3 Near the Receiver .............................................................................. 106

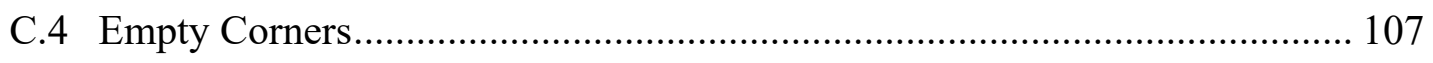

Appendix D Samples of Data with Different Device Placements .......................... 109

D.1 2D (Ground-Ground) Device Placement................................................ 109

D.2 3D (Ceiling-Ground) Device Placement ............................................ 110

Appendix E Samples of Data by Different Subjects........................................... 111

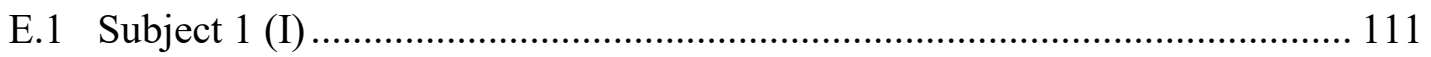

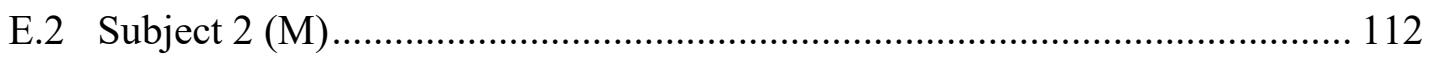

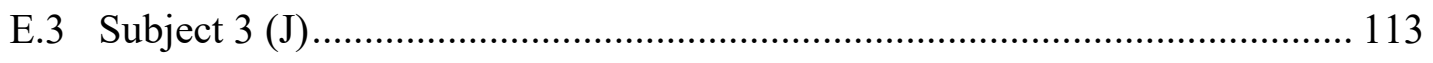

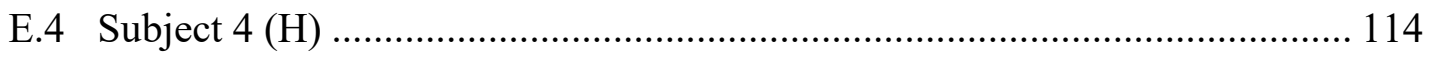

Bibliography or References............................................................................................... 115 


\section{List of Tables}

Table 1 Cameras vs. Sensors vs. Wi-Fi vs. Radar ................................................... 7

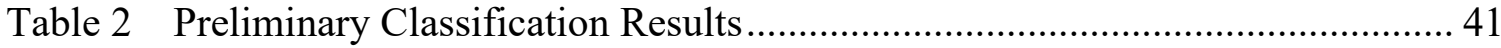

Table 3 Confusion Matrix of the Medium Gaussian SVM for the "preliminary" Data

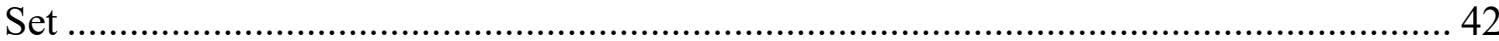

Table 4 Comparison of Classification Accuracies achieved by Normalization

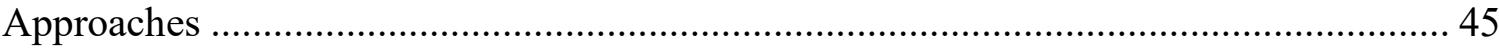

Table 5 Comparison of Classification Accuracies achieved by Feature Scaling

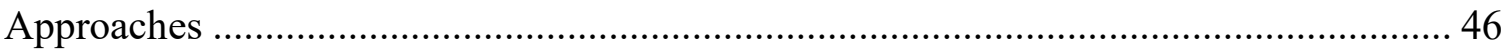

Table 6 Classification Results with Optimum Preprocessing Methodology ................ 47

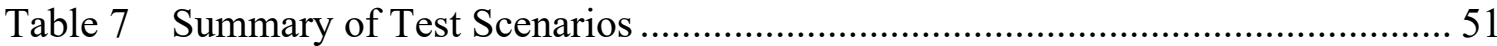

Table 8 Classification Results Demonstrating the Independence of Performance with

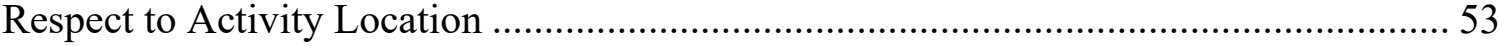

Table 9 Device Placement Classification Results....................................................... 55

Table 10 Confusion Matrix of the Medium Gaussian SVM for the "preliminary" Data

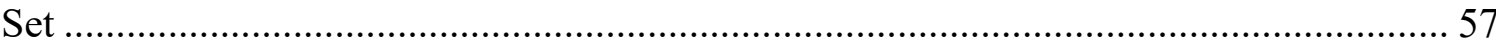

Table 11 Confusion Matrix of the Medium Gaussian SVM for the "floor" Data Set ... 57

Table 12 Confusion Matrix of the Medium Gaussian SVM for the "ceiling" Data Set 58

Table 13 Confusion Matrix of the Medium Gaussian SVM for the "floor + ceiling"

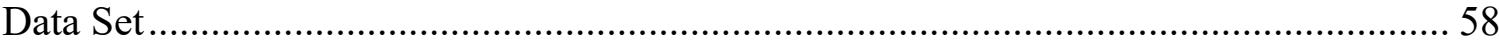

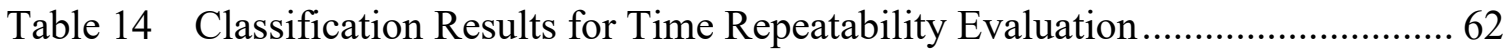


Table 15 Confusion Matrix Summarizing the Medium Gaussian SVM Predictions for "day 1" 63

Table 16 Confusion Matrix Summarizing the Medium Gaussian SVM Predictions for "day 2" 63

Table 17 Classification Results showing Time Repeatability on Two Data Sets from the Same Day. 64

Table 18 Confusion Matrix Summarizing the Medium Gaussian SVM Predictions for Capture 1 65

Table 19 Confusion Matrix Summarizing the Medium Gaussian SVM Predictions for Capture 2

Table 20 Confusion Matrix Summarizing the Medium Gaussian SVM Predictions for

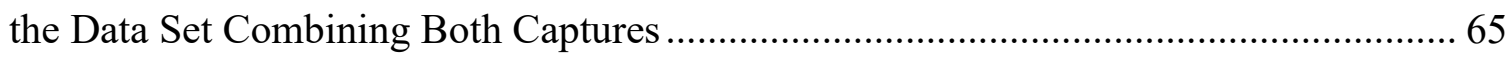

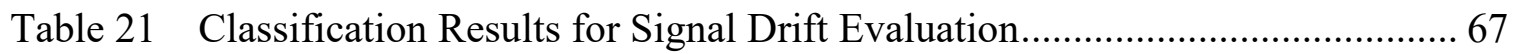

Table 22 Classification Results showing Signal Drift on Two Data Sets from the Same

Day. 69

Table 23 Subject Information and Characteristics ............................................. 70

Table 24 Classification Results for Individual Subjects, Dependent on the Subject..... 72

Table 25 Confusion Matrix Summarizing the Medium Gaussian SVM Predictions for

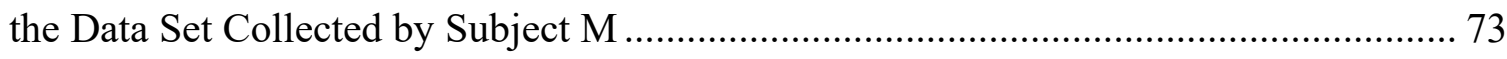

Table 26 Confusion Matrix Summarizing the Medium Gaussian SVM Predictions for the Data Set Collected by Subject J 73

Table 27 Confusion Matrix Summarizing the Medium Gaussian SVM Predictions for the Data Set Collected by Subject H 
Table 28 Confusion Matrix Summarizing the Medium Gaussian SVM Predictions for the "other" Data Set, Combining Data Collected by Subjects M, J, and H.................... 74

Table 29 Classification Results of Training of Different Data Sets, Independent of the

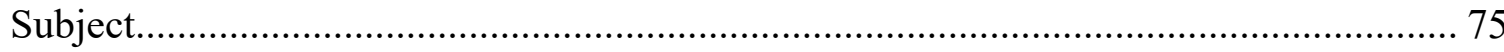

Table 30 Classification Results of Training on "floor" Data Set and Testing on "other"

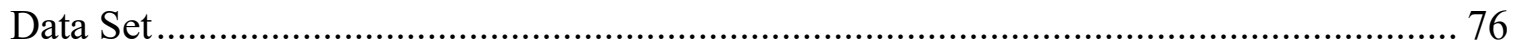

Table 31 Classification Results of Training on "ceiling” Data Set and Testing on

"other" Data Set

Table 32 Classification Results of Training on "floor + ceiling" and Testing on "other"

Data Set.......

Table 33 Confusion Matrix Displaying the Validation Predictions of a Sequence-to-

Label BiLSTM Network, with Three Activity Classes ............................................ 82

Table 34 Confusion Matrix Displaying the Validation Predictions of a Sequence-to-

Label BiLSTM Network, with Four Activity Classes ............................................ 83

Table 35 Summary of Results from Both Sequence-to-Sequence and Sequence-to-Label

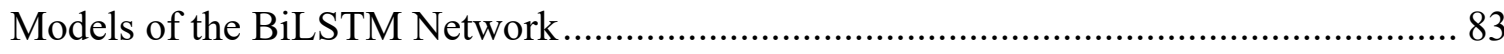

Table 36 Comparison of Classification Performances with Related Work ................. 85

Table 37 Summary of Sitting and Standing Data Samples Collected under a 2D

(Ground-Ground) Device Placement, by the Author ................................................ 91

Table 38 Summary of Sitting and Standing Data Samples Collected under a 3D

(Ceiling-Ground) Device Placement, by the Author 92

Table 39 Summary of Sitting and Standing Data Samples Collected by Three Volunteer Subjects 92 
Table 40 Summary of Additional Data Samples Collected by the Author, but Not Used 


\section{List of Figures}

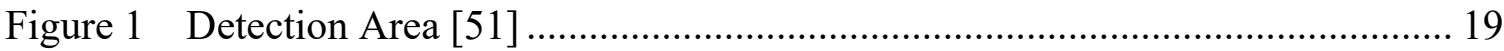

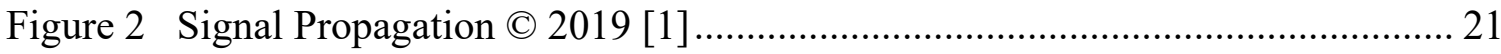

Figure 3 Deployment Topology and Components ………………………………...... 22

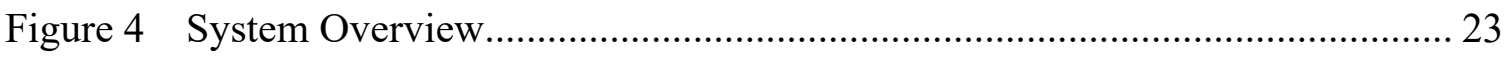

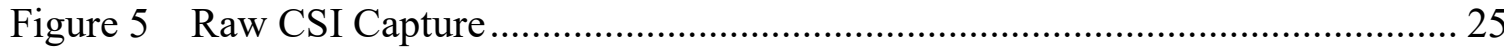

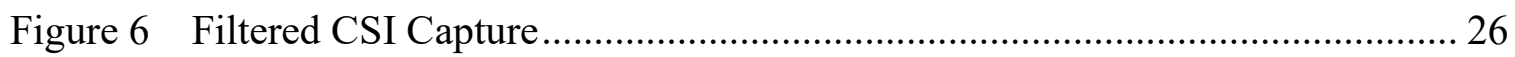

Figure 7 Sample Time Series of Subcarrier Features (C) 2019 [1] ................................. 28

Figure 8 Sample Plots for Moving Variance of the Mean and of the Variance (C) 2019

[1]

Figure 9 The Three Levels of Extracted Features....................................................... 30

Figure 10 Layers of Designed Neural Network …………………............................... 36

Figure 11 Initial activity location with the chair placed in the middle of the room $($ C

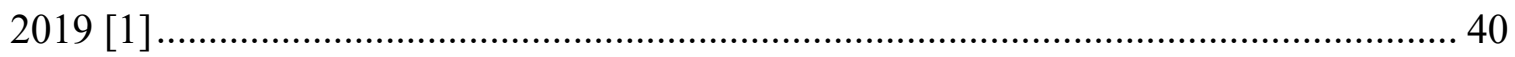

Figure 12 Top Views of Experimental Setups .......................................................... 49

Figure 13 Classification Accuracy per Classifier for Device Placement....................... 56

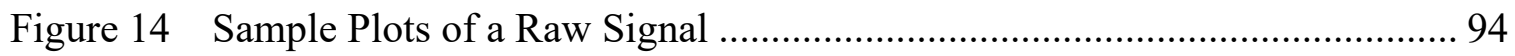

Figure 15 Sample Plots of a Filtered Signal ............................................................. 95

Figure 16 Sample Plots of Signals Normalized via Z-Score Standardization, over the

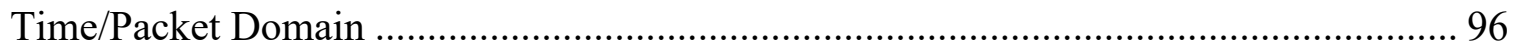

Figure 17 Sample Plots of Signals Normalized via Z-Score Standardization, over the

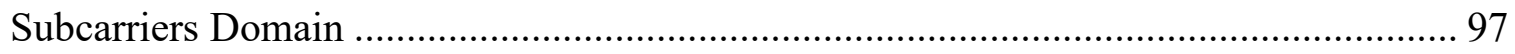


Figure 18 Sample Plots of Signals Normalized using a Fixed-Factor, over the Time/Packet Domain 98

Figure 19 Sample Plots of Signals Normalized using a Fixed-Factor, over the Subcarriers Domain 99

Figure 20 Sample Plots of Signals Scaled through Subcarrier Features..................... 100

Figure 21 Sample Plots of Signals Scaled through Time Features ............................ 101 Figure 22 Sample Plots of Signals Scaled through Both Subcarrier and Time Features 102

Figure 23 Sample Plots of Filtered, Z-Score Standardized (over subcarriers), and Scaled

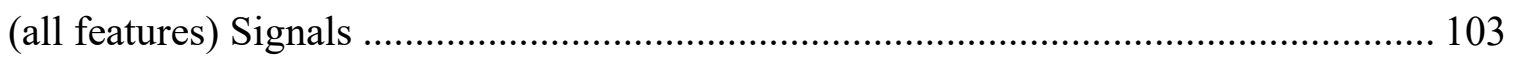

Figure 24 Sample Plots of Data Collected at the Middle of a Room......................... 104

Figure 25 Sample Plots of Data Collected near the Transmitter ............................... 105

Figure 26 Sample Plots of Data Collected near the Receiver ................................. 106

Figure 27 Sample of Plots of Data Collected at the Empty Front-Left Corner of a Room 107

Figure 28 Sample of Plots of Data Collected at the Empty Back-Right Corner of a Room 108

Figure 29 Sample of Plots with Data Collected under a 2D (Ground) Device Placement 109

Figure 30 Sample of Plots with Data Collected under a 3D (Ground-Ceiling) Device Placement. 110

Figure 31 Sample of Plots for Data Collected by Subject 1 ................................. 111

Figure 32 Sample of Plots for Data Collected by Subject 2 ................................. 112 
Figure 33 Sample of Plots for Data Collected by Subject 3 .................................. 113

Figure 34 Sample of Plots for Data Collected by Subject 4 ................................. 114 


\section{List of Appendices}

Appendix A Summary of Data Samples ....................................... 91

Appendix B Samples of Preprocessed Data ......................................... 94

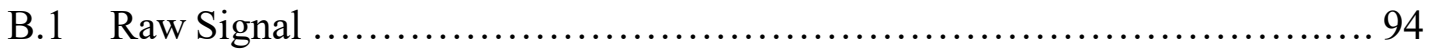

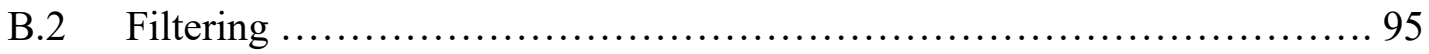

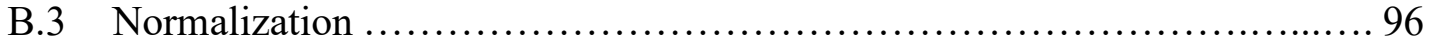

B.4 Feature Scaling ...................................................... 100

B.5 Filtering, Z-Score Standardization, and Feature Scaling Combined ............ 103

Appendix C Samples of Data from Different Activity Locations ....................... 104

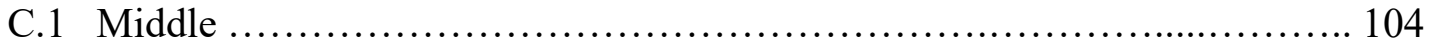

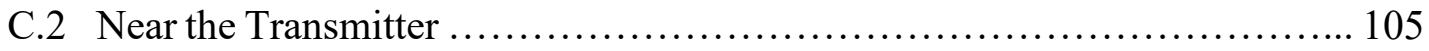

C.3 Near the Receiver ..................................................... 106

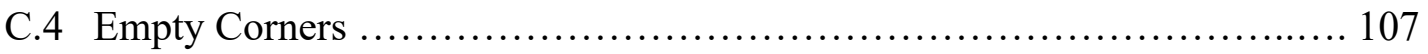

Appendix D Samples of Data with Different Device Placements ..................... 109

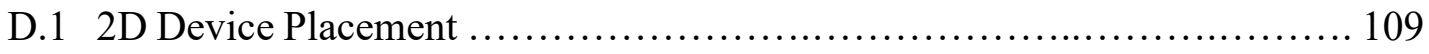

D.2 3D Device Placement ................................................. 110

Appendix E Samples of Data by Different Subjects ............................... 111

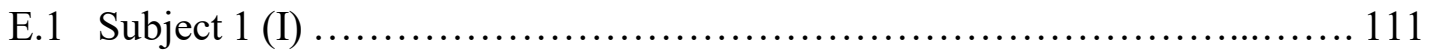

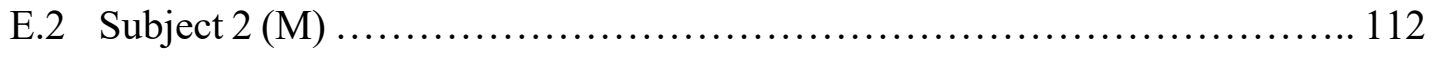

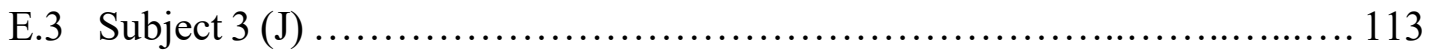

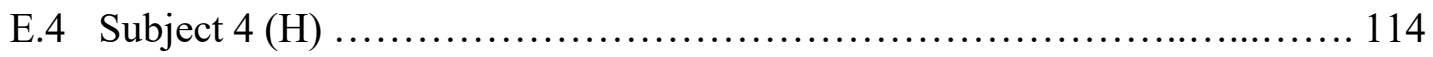




\section{Table of Acronyms}

\begin{tabular}{|l|l|}
\hline Acronym & Term \\
\hline 1D & One-Dimensional \\
\hline 2D & Two-Dimensional \\
\hline 3D & Three-Dimensional \\
\hline AAL & Ambient Assistive Living \\
\hline ABLSTM & Attention-based Bidirectional Long Short-Term Memory \\
\hline ADAM & ADAptive Moment Estimation \\
\hline ADASYN & Adaptive Synthetic Sampling \\
\hline ADL & Activities of Daily Living \\
\hline AP & Access Point \\
\hline API & Application Programming Interface \\
\hline BiLSTM & Bidirectional Long Short-Term Memory \\
\hline CNN & Convolutional Neural Network \\
\hline CSI & Channel State Information \\
\hline DA & Discriminant Analysis \\
\hline DT & Decision Tree \\
\hline F1 & F-Score \\
\hline FN & False Negatives \\
\hline FNN & Feedforward Neural Network \\
\hline FP & False Positives \\
\hline HAR & Human Activity Recognition \\
\hline HMM & Hidden Markov Model \\
\hline IEEE & Institute of Electrical and Electronic Engineers \\
\hline KNN & K-Nearest Neighbours \\
\hline LAN & Local Access Network \\
\hline MIMU & Magnetic and Inertial Measurement Unit \\
\hline MLP & Multi-Layer Perceptron \\
\hline MVMN & MultiVariate MultiNomial \\
\hline NB & Naïve Bayes \\
\hline NSERC & Natural Sciences and Engineering Research Council \\
\hline OFDM & Orthogonal Frequency Division Multiplexing \\
\hline RF & Radio Frequency \\
\hline RSS & Received Signal Strength \\
\hline SCE & Systems and Computer Engineering \\
\hline SMOTE & Synthetic Minority Oversampling TEchnique \\
\hline STA & Client Station \\
\hline SVM & Support Vector Machine \\
\hline TN & True Negatives \\
\hline TP & True Positives \\
\hline WLAN & Wireless Local Area Network \\
\hline & \\
\hline
\end{tabular}




\section{Chapter 1: Introduction}

This chapter details the motivation behind this thesis, reviews the problem statement, states the objectives and contributions, and describes the structure of the thesis.

\subsection{Motivation}

Real-time monitoring and recognition of human activities is an essential functionality

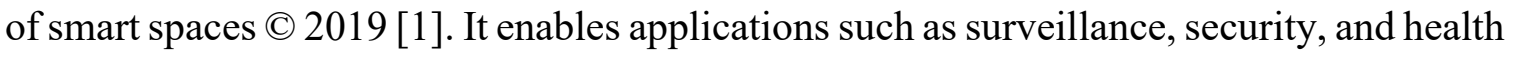
monitoring to assist living for older adults and/or for people with disabilities. Establishing

an accurate Human Activity Recognition (HAR) system, which is non-intrusive and passive, would facilitate smart spaces by allowing older adults to stay in the comfort of their own homes and live independently; hence, improving their well-being, while significantly reducing the healthcare costs associated with admission to long-term care [2]. It could also help and support people with disabilities in their everyday activities. A HAR system would also be useful for generating alerts to the user, a patient's family, and/or caregivers should abnormal activities be identified.

\subsection{Objectives}

The main objective of this thesis is to remotely analyze and recognize human activities using the Wi-Fi devices, supplied by Aerial Technologies, in order to provide reliable means of health monitoring to assist living for older adults or people with disabilities. The main research question that this thesis tries to answer is whether Wi-Fi can be utilized to distinguish between human Activities of Daily Living (ADLs). The work was limited to sitting-down and standing-up activities in order to manage the expectations of this thesis. 


\subsection{Problem Statement}

Human ADLs, such as bathing, cooking, eating, driving, resting (sleeping or sitting), etc., describe our behavioural and functional abilities. The monitoring and recognition of ADLs can significantly help to diagnose and assess many health problems that relate to aging or mental states. They can also be used in assistive living applications to support the vulnerable population (elderly and disabled). Human activities can be assessed via various means of measurement, such as wearable sensors, surveillance cameras, radar, and Wi-Fi. Wearable sensors must be worn by the user, which can be uncomfortable. On the other hand, video can be a significant intrusion on privacy when recorded. As such, this leads to a need for an alternative solution that assesses ADLs through ambient sensing, such as Radio Frequency (RF), where radar is one option and Wi-Fi is another. This work focuses on the potential for RF, specifically the use of low-cost Wi-Fi, to establish a passive and non-intrusive HAR system to identify and recognize ADLs.

\subsection{Contributions}

This thesis focuses on classifying sitting-down and standing-up activities to specifically distinguish them from each other and from a no-motion, still case. The thesis was conducted in several stages of research. As indicated below, some of the contributions made in this thesis have been published:

1. Performing data collection, preprocessing, visualization, and analysis in order to prove that Wi-Fi can be used to distinguish between the two similar motions of sitting-down and standing-up actions [1];

2. Proposing a novel system for classifying human activities based on Wi-Fi data by leveraging traditional machine learning solutions such as decision 
trees, discriminant analysis, naïve Bayes, Support Vector Machines (SVMs), K-Nearest Neighbours (KNN), or ensemble classifiers to recognize human activities [1];

3. Exploring multiple preprocessing techniques to boost the classification performance of the system [1];

4. Validating the proposed solution for location and participant movement independence by assessing varying sitting and standing speeds and styles at different room locations;

5. Studying the impact of the location of Wi-Fi sensor devices on the classification performance, through the addition of a vertical dimension to the measurements, where one sensor was installed up at the ceiling and the other down on the ground to include elevation information in the measurements;

6. Evaluating the capability of time repeatability on the classification performance to test the time independence of the proposed solution;

7. Investigating the possibility of eliminating the need for repetitive training, in the case of environmental changes or subject motion, by pre-training the classifiers on a wide range of data samples from a single person, and testing them on a diverse population of volunteers to cover various ages and genders, as well as under different environmental settings to cover varying distances between access points (APs); and

8. Designing and implementing a neural network, and leveraging it to apply two deep learning models of classification: sequence-to-label and sequence-tosequence. 


\subsection{Thesis Structure}

This thesis is organized into six chapters. The next three chapters give a brief background about the topic and outline the used methodology and the proposed solution, respectively. Chapter 2 provides a brief background on the topic. Chapter 3 outlines the used methodology. The remainder of the thesis discusses the work carried out to achieve contributions 1 through 8, listed in section 1.4. As such, Chapter 4 introduces the proposed

solution, while the experimental results are presented in Chapter 5. Finally, Chapter 6 concludes the thesis. 


\section{Chapter 2: Background}

This chapter provides a quick overview of the required knowledge to understand the context of this thesis, and briefly covers relevant state-of-the-art work in this context.

\subsection{Activities of Daily Living (ADLs)}

ADLs define our daily routines. There are two types of ADLs: basic and instrumental [3]. Basic ADLs include hygiene activities such as dressing, bathing, self-feeding, and basic kitchen use. Instrumental ADLs are more complex tasks that are composed of multiple steps or require the use of instruments, such as food preparation, shopping, driving, social activities, and using a computer. There has always been a challenge for the accurate reporting of functional abilities based on patients' histories of performing ADLs. Clinicians use changes in the ability to perform ADLs to diagnose and classify the severity of diseases or decline through remote episodic assessments of day-to-day activities, which could help identify behavioural and functional changes. Today, clinicians solely rely on self-reporting, but there is a potential for episodic assessments to be automated. This can be accomplished by monitoring and detecting the ADLs of patients on an ongoing basis using sensor solutions. This thesis shows the potential of achieving this through Wi-Fi sensors versus solely relying on self-reports.

For a typical person, the importance of the monitoring and recognition of ADLs might not be as intuitive. This, however, becomes crucial when it comes to the vulnerable population (i.e. seniors and persons with disabilities). The effects of aging and illness can cause older adults to need support and assistance from a family member or a care giver. The decline can include changes in physical and/or cognitive abilities. Persons with 
disabilities may need special accommodations to support them in completing their ADLs. The context of this work focuses on the elderly, in an initial attempt to establish a passive and non-intrusive HAR system to identify and recognize ADLs.

\subsection{ADL Assessment Technologies}

The assessment of ADLs is currently based on self-reporting. This might not be an accurate approach, as patients might forget or deny. Hence, there is a need to assess ADLs via a more reliable mechanism of sensing, such as image/video, sensors, and RF. The choice of these technologies is driven from both human factors, and their usability by the individual as well as the usability of the technology to process the sensor data in order to achieve accurate results.

Game playing is an example of a complex task that could be a proxy for the assessment of instrumental ADLs [3]. Games that are easy to learn, play, and are age appropriate for older patients can potentially be used to measure the cognitive well-being of patients [3 - 5]. For instance, a whack-a-mole game is used to identify changes in cognitive abilities for older adults [4, 5].

\subsubsection{Usability of ADL Sensing Options for Aging Adults}

Activity monitoring and recognition systems have been reported to utilize cameras [6 - 15] and/or other types of sensors [16 - 26]. The raised usability issues of sensors include the discomfort of users, the requirement for a person to carry them at all times, and the need for the regular charging of devices $\mathbb{C} 2019$ [1]. Camera-based systems have been reported with privacy issues, raising concerns regarding their use in spaces like bathrooms or bedrooms [6 - 15]. Unlike these, a Wi-Fi-based approach has the potential to be 
convenient, independent of environmental changes, such as lighting, and eliminates the privacy issues associated with video. Since Wi-Fi is pervasive and low cost, this approach reduces deployment costs in contrast to alternative RF-based solutions, such as radar, which can give accurate results, but is very costly. Table 1 compares the advantages and disadvantages of four technologies: cameras, sensors, Wi-Fi, and radar.

Table 1 Cameras vs. Sensors vs. Wi-Fi vs. Radar

\begin{tabular}{|c|c|c|c|}
\hline Cameras & Sensors & Wi-Fi & Radar \\
\hline privacy concerns & uncomfortable & non-intrusive & non-intrusive \\
\hline requires lighting & integration and calibration & passive & passive \\
\hline battery or electricity & battery or electricity & electricity & electricity \\
\hline expensive & cheap & pervasive & expensive \\
\hline
\end{tabular}

\subsubsection{ADL Assessment with Cameras}

Cameras are one possible solution for ADL monitoring. Cameras are more commonly used for surveillance purposes $[6,7]$, rather than health monitoring. It would be odd for them to be used for healthcare applications in a private home setting, especially in more private spaces like bathrooms and bedrooms. However, the use of cameras has been considered in less private areas, such applications including respiration rate estimation [8], gesture recognition [9], gait analysis [10], human activity recognition [11 - 15]. Most stateof-the-art studies apply machine learning in their solutions. A novel video surveillance system that uses one static camera was presented in [6] for human posture recognition through K-means and fuzzy C-means clustering, Multi-layer Perceptron (MLP) selforganizing maps, and Feedforward Neural Networks (FNNs). An artificial neural network [7] was designed to perform human activity monitoring and detection of suspicious activities within a surveillance system. 
An approach to estimate inhale and exhale durations as well as their ratios using a video stream, from the upper torso, was proposed in [8]. A study that uses the SVM, MLP, and naïve Bayes classification techniques [9] was conducted to recognize gestures of the human body, captured via a Kinect camera. A method that measures relative gait parameters during unconstrained straight-line walking using a single camera was proposed in [10] to estimate step length ratios.

A novel space-boundary detection method that leverages a mobile camera and a finite state machine [11] was employed to identify the location and pose of a person within a home setting, in order to improve the human-robot interaction for health care and companion applications. A stereo camera was used to acquire activity video images of the body joint angles for a three-dimensional (3D) HAR system that generated a Hidden Markov Model (HMM) of activities [12]. Moladande et al. [13] proposed a novel approach that uses verbal communication, behaviour recognition, and motion recognition to identify user intentions via machine learning, computer vision, and voice recognition for human robot interaction in assistive technologies. In [14], a moving camera was used to capture human activities with background areas that undergo diverse camera motions (i.e. motionplanes and multiple homographies) for activity localization and recognition. A technique that depends on multi-view videos, motion frequency features, and SVM [15] was proposed to achieve activity recognition.

\subsubsection{ADL Assessment with Sensors and Smartphones}

Numerous authors have been performing research on the topic of activity recognition using sensors and/or smartphones, such as monitoring for overnight wandering by a person with dementia [16] using low-cost motion, bed, and latch sensors, as well as 
speakers, and smart lights (C 2019 [1]. A study on physiological postural monitoring [17] using low-cost, easy-to-integrate temperature sensors, as opposed to pressure sensors, showed that temperature changes are correlated with posture changes. This work was extended in [18] to present two alternative instrumentation and measurement means to find chair occupancy, postural change timings, and posture states of a person in a chair.

A custom threshold-based hierarchical decision tree that performs transition state identification and context awareness [19], was proposed to classify mobile and immobile states of human activities using accelerometer, magnetometer, and gyroscope data from smartphones@ 2019 [1]. Five different classifiers: KNN, FNN, SVM, naïve Bayes, and decision trees, were compared [20] for their performance on recognizing sitting, standing, laying, ascending and descending stairs, and walking activities using a Magnetic and Inertial Measurement Unit (MIMU) sensor containing a tri-axial accelerometer, gyroscope, and magnetometer. A real-time algorithm [21] was proposed to differentiate between falls, sitting, standing, laying, ascending and descending stairs, and walking activities using a smart sensor, which is based on a tri-axial accelerometer, connected to a microcontroller Wi-Fi module. A smartphone-based ADL detector [22] was developed to provide reliable monitoring for the elderly in the context of Ambient Assistive Living (AAL) through two algorithms: 1) threshold-based classification, and 2) principal component analysis-based classification to distinguish falls from other events. This work was later extended in [23], and again in [24] to apply a multisensory data-fusion paradigm. A method of equestrian analysis based on inertial body sensor networks [25] was proposed to help correct horseriding postures. A non-iterative orientation estimation method, based on the physical and geometrical properties of the acceleration, angular rate, and magnetic field vectors was 
used in [26] to estimate the orientation of motion sensor units for the recognition of daily and sports activities.

\subsubsection{ADL Assessment with Radar}

Other studies considered the use of radar sensors to assess ADLs. A fall detection system that uses a $79 \mathrm{GHz}$-band millimeter wave sensor (i.e. radar) was proposed in [27] to detect possible bathroom falls (C) 2019 [1]. It utilizes k-means clustering to analyze the motion of a bathing person. A wireless sensor network that uses the generative HMM and discriminative conditional random fields [28] was proposed to recognize activities from non-intrusive observations. Gu et al. [29] distinguished among hand gestures from interfering movements using a solution based on a single-input multiple-output frontend and a blind motion separation algorithm, through continuous-wave radar sensors. In [30], a Convolutional Neural Network $(\mathrm{CNN})$ was utilized to classify digits written in the air via hand gestures, where the hand gestures are captured using impulse radio ultra-wideband radar sensors.

\subsubsection{ADL Assessment with Wi-Fi}

The main principle of Wi-Fi human activity monitoring uses the strength, amplitude, and phase of signals between two points of communication (i.e. transmitter and receiver) as they are affected by human movements (C) 2019 [1]. Modern wireless communications use Orthogonal Frequency Division Multiplexing (OFDM) [31] to encode digital data on multiple carrier frequencies. A Wi-Fi signal propagates between a transmitter and a receiver through multiple transmission channels. The transmitter broadcasts simultaneously on several narrowly separated subcarriers, within each channel, to increase the data rate. Thus, the information obtained from the physical layer of wireless 
infrastructures, such as Channel State Information (CSI) and Received Signal Strength (RSS), can potentially be used to characterize human activities.

CSI describes how the transmitted signal is propagated through the channels and is measured for each OFDM subcarrier, providing amplitude and phase responses for each subcarrier [31] @ 2019 [1]. In an indoor environment, the CSI signal remains stable for an unchanging room. Once a person is present, the CSI signal starts reflecting amplitude and phase alterations, due to different multipath effects, based on movements [31]. So, CSI can be utilized to recognize ADLs.

Recent studies leveraged Wi-Fi signals for human identification [32] and localization [33 - 36], intruder detection [33, 36], vital signs monitoring [37 - 39], gesture tracking [40, 41], and basic activity recognition [35, 42 - 47] @ 2019 [1]. WiWho [32] is a system that can identify a person from a small group of two to six people using the CSI of Wi-Fi signals. It performs CSI preprocessing to remove distant multipath and highfrequency noises, and uses a decision tree-based approach to classify people's gait pattern profiles. A real-time trajectory tracking technique that uses mobility HMMs and the received power of Wi-Fi signals (i.e. RSS) was proposed in [34] to localize people in an indoor environment. The authors of [35] applied a radio image processing method to characterize the influence of human location and activity on Wi-Fi CSI via a deep learning network. In this study, CSI measurements from multiple Wi-Fi channels were transformed into a radio image and interpreted as two-dimensional (2D) data. An initial attempt for outdoor human detection through IEEE 802.11ac Wireless Local Area Network (WLAN) CSI was presented in [36]. 
Liu et al. [37] proposed a system that monitors vital signs, such as respiration rate and heart rate, during sleep using CSI amplitude and phase measurements (C) 2019 [1]. PhaseBeat [38] uses CSI phase information to monitor and estimate respiration rate and heart rate. Wi-Fi's RSS was also exploited to locate breathing persons and estimate their breathing rate, while sitting, laying down, standing, or sleeping, without calibration [39].

WiTrace [40] is a Wi-Fi CSI-based centimeter-level one-dimensional (1D) and 2D tracking system for hand gestures (C) 2019 [1]. WiKey [41] is a system that exploits the CSI of Wi-Fi signals to recognize keystrokes by analyzing the unique formations and directions associated with such hand and finger gestures.

E-eyes [42] is an activity recognition system that uses CSI to distinguish between in-place and walking activities. CSI measurements were preprocessed to eliminate outliers, and a moving variance was applied to determine the category of the activity (C) 2019 [1]. A large moving variance represents a walking activity, while a small moving variance represents in-place or no activity. E-eyes performs location-oriented identification of inplace and walking activities, with an average accuracy of 92\%. CARM [43] is a quantitative activity monitoring and recognition system that associates CSI value dynamics with human movements and activities through two models: a CSI-speed model, and a CSIactivity model. As a HAR system, CARM can achieve an average recognition accuracy of 96\%. A multi-layer CNN [44] was developed to analyze and classify the CSI data acquired from multiple Wi-Fi APs in order to recognize standing, sitting, and hand moving/raising activities. The CNN achieves an accuracy of over $80 \%$ for most tested cases. Doppler frequency shifts in CSI [45] were proposed to recognize human behaviours such as picking up an item from the floor, sitting, standing, falling, as well as getting up and out of bed in 
an indoor environment. The approach proposed in [45] achieves accuracy at recognizing dynamic sitting-down and standing-up movements was $92 \%$. This was then extended to detect human presence and classify standing and walking events [46]. The Attention-based Bidirectional Long Short-Term Memory (ABLSTM) approach [47] was proposed for the recognition of laying, falling, walking, running, sitting, standing, jumping, picking up, and hand waving activities. The ABLSTM network achieves an accuracy of $95 \%$ at recognizing sit-down activities, and $98 \%$ at recognizing stand-up activities. It has a probability of $52 \%$ at misclassifying sit-down activities as stand-up activities.

Finally, as part of a project done in collaboration between Aerial Technologies, Inc. and McGill University, Ghourchian et al. [33] proposed a Wi-Fi-based indoor localization system, which uses random forests to locate a subject and detect intruders in a residential unit in the context of motion detection in home security applications (C) 2019 [1]. The state-of-the-art shows that machine learning algorithms can be used to associate the obtained CSI signals with ADLs.

\subsection{Machine Learning}

ADLs can be identified and recognized through various means, such as mathematical modelling $[8,10,21,25,26,29,38,40,41,43]$, machine learning $[6,9,13,19,20,22$ $24,27,28,30-35,42,44,46,47]$, or a combination of both [7, 11, 14, 15]. Mathematical models and algorithms can be quite time consuming, as a lot of data analysis is required to derive them. On the other hand, machine learning approaches can simplify such analysis, as they are used to automate the modelling process, by studying the patterns associated with the data $[48,49]$. 


\subsubsection{Training}

As the name suggests, machine learning is a process in which a machine learns to perform a task using a set of training data [48, 49]. Machine learning processes can be supervised or unsupervised. Supervised learning requires the inputted training data to contain information about the correct output. In unsupervised learning, the machine determines the output to a given problem based on the associated patterns.

Any supervised machine learning process consists of a classifier that is trained on a data set of labelled attributes/variables/features to fit into the classifier's model $[48,49]$. The trained classifier can then be utilized to recognize other data samples, which are unlabelled, by accordingly predicting the classes they belong to.

Examples of supervised learning algorithms include decision trees, discriminant analysis, naïve Bayes, SVMs, and KNN [48]. Decision trees are constructed from conditional statements based on the boundaries in the variables of the inputted training data set, where each branch represents a condition and outputs a decision. Discriminant analysis is the process of distinguishing between two or more classes using a boundary function. Naïve Bayes classification depends on a collection of probabilistic algorithms that are based on Bayes' theorem, which views each feature independently. SVMs attempt to identify the optimal hyperplane for categorizing data samples. An optimal hyperplane defines a clear gap between the data points in terms of space. The KNN algorithm determines the class of a sample according to its $k$ nearest neighbours. 


\subsubsection{Testing and Validation}

It is essential to validate the learning process to avoid problems like classifier overfitting, which occurs when the learner memorizes the training data [48]. In other words, overfitting is the outcome of perfectly fitting the model into the training data, causing it to fail at recognizing novel data samples, that were not presented during the training. Another problem that is common in machine learning classification is class imbalance, which is the phenomenon of having a data set that contains a lot more samples of one class (majority class) than the other class (minority class). Class imbalance can be resolved by 1) discarding the excess samples of the majority class, 2) obtaining more samples of the minority class, 3) oversampling and undersampling, or 4) synthesizing new data samples.

Training results can be validated by evaluating the trained model against a testing data set, which consists of a portion of the original training data set that is not included in the training process, or a totally different data set that contains similar samples [48]. There are several validation methods that can be used in machine learning, such as holdout testing and k-fold cross validation, among many other techniques. Holdout testing is the process of splitting the data into a training data set and a testing data set, where a larger portion of the data is kept for training and the rest is held out for testing at a later stage. To avoid overfitting in holdout testing, an equal distribution of the different classes in the data is usually created. Holdout testing is preferred when the training data set is large and contains imbalanced classes. K-fold cross validation is the process of repeatedly training the classifier on $k-1$ folds (i.e. partitions) of the data, while testing it on the remaining fold. It is a form of iterative holdout testing, where the results are averaged across $k$ iterations 
to determine the final decision. This is good to continually evaluate the capability of the classifier to recognize new, unseen data, and thus, helps to avoid overfitting. In this work, 5-fold cross validation was used to protect the classifiers against overfitting.

\subsubsection{Performance Metrics}

The performance of a classifier can then be measured by comparing the predicted classes with the true classes of the data samples [48]. There are different metrics that can be calculated to measure the performance of a classifier: accuracy, precision, sensitivity, specificity, and F-score. In the case of binary classification, such metrics can be determined using the number of true positives (TP), true negatives (TN), false positives (FP), and false negatives $(\mathrm{FN})$.

True positives are the samples that are classified positive and actually belong to the positive class, while true negatives are the samples that are classified negative and actually belong to the negative class [48]. False positives are the samples that are classified positive but belong to the negative class, while false negatives are the samples that are classified negative but belong to the positive class. Accuracy is the measure of correctly classified samples (i.e. rate of true samples among all samples), that is:

$$
\text { Accuracy }=\frac{T P+T N}{N}
$$

where $\mathrm{N}$ is the total number of samples. Precision measures the correctness in positive predictions (i.e. rate of TP among all positive predictions), that is:

$$
\text { Precision }=\frac{T P}{T P+F P}
$$


Sensitivity is the measure of correctly classified positives (i.e. true positive rate), whereas specificity is the measure of correctly classified negatives (i.e. true negative rate), that is:

$$
\begin{aligned}
& \text { Sensitivity }=\frac{T P}{T P+F N} \\
& \text { Specificity }=\frac{T N}{T N+F P}
\end{aligned}
$$

The F-score (F1) is the harmonic mean of precision and sensitivity, which can be calculated as follows:

$$
\begin{aligned}
F 1 & =2 \cdot \frac{\text { precision } \cdot \text { sensitivity }}{\text { precision }+ \text { sensitivity }} \\
& =\frac{2 T P}{2 T P+F P+F N}
\end{aligned}
$$

A confusion matrix is usually used to summarize TP, TN, FP, and FN. The rows of a confusion matrix represent the true class (e.g. activity) of the data samples, whereas the columns represent the class that the learner predicted, accordingly.

In multiclass classification problems, one can use true and false ratios for each class instead of limiting the ratios to TP, TN, FP, and FN, for a total of $n \times 2$ ratios, where $n$ represents the number of classes. The performance metrics can then be calculated using the true and false ratios of all classes.

\subsection{Deep Learning}

Deep learning is an advanced type of machine learning. Deep learners are neural networks that are deep and have many neurons [50]. Deep learning models are usually 
composed of multiple levels of abstraction, where the backpropagation algorithm is used to determine the amount of adjustment in a machine's parameters from one level to the next. They have the potential to analyze patterns associated with complex forms of data. Deep learning models can be trained on data sets of images, videos, sequences, or time series. Hence, they enable complex applications, such as speech recognition, face recognition, and activity recognition. 


\section{Chapter 3: Equipment}

This chapter describes the equipment used to accomplish the contributions proposed in this thesis.

\subsection{Theory of Operation of the Equipment}

This work was developed in collaboration with industry. Aerial Technologies supported the work by providing their platforms for motion detection to determine if they could also accomplish human activity recognition. Aerial's motion detection technology leverages the patterns in Wi-Fi signals propagating between two connected Wi-Fi devices (transmitter and receiver) to analyze and detect the intensity of motion within an area of coverage [51]. The detection then occurs in the range of an ellipsoid around the two connected Wi-Fi devices, acting as the focal points. The highest motion intensities are detected when moving near the devices or in the direct path between the two devices. Although the intensity of motion reduces at other positions, motion would still be detected as long as movements are happening in the coverage area (i.e. ellipsoid shape in Figure 1).

Figure 1 illustrates an example of a detection area.
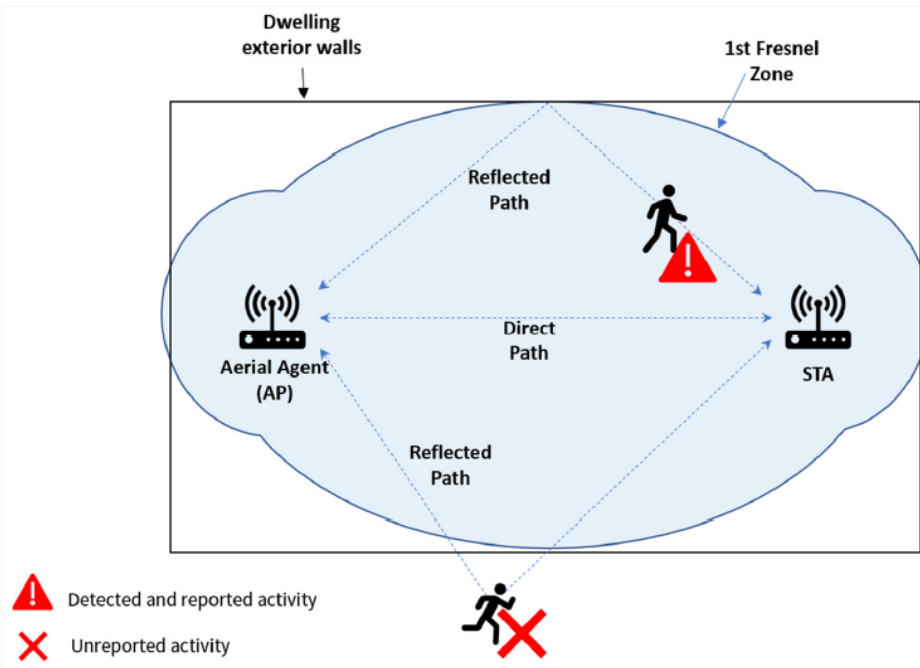

Figure 1 Detection Area [51] 
The size and shape of the detection area can be altered by many factors, such as the shape, size and layout of the place; furniture and obstacles; building materials; as well as the distance between the Aerial devices [51]. The detection area increases and decreases with the distance between the devices. The two Wi-Fi devices consist of an AP and a client station (STA) in Figure 1, each having a different functionality. These devices are designed and tested in a laboratory environment, and they have no shielding. Hence, they are affected by electromagnetic interference when placed within a small proximity of other wireless devices and/or machines, such as computers.

The maximum detection area that can be covered by the Aerial devices is approximately 1,200 square feet (110 square meters) [51]. A single AP-STA pair might not be enough to guarantee complete coverage of larger spaces, in which case, multiple APSTA pairs may be used. Also, it is highly recommended to place the devices in the most visited areas such as entrances, hallways, living rooms, etc. for better coverage, with fewer devices.

\subsection{Working Principle of the Equipment}

Aerial Technologies provides an AP and a STA that can collect CSI data according to the IEEE 802.11n and IEEE 802.11ac standards [51]. The AP acts as the transmitter, whereas the STA acts as the receiver. Each device contains four antennas, which results in 16 potential active streams. Figure 2 shows the manner in which the Wi-Fi signal is propagated from the transmitter to the receiver, having a direct path and multipath components due to reflections and diffractions on objects and walls. Each spatial stream contains multiple subcarriers in the frequency domain. The sampling rate of the system is pre-set to 20 packets or samples per second, while the bandwidth is pre-set to 80 megahertz. 


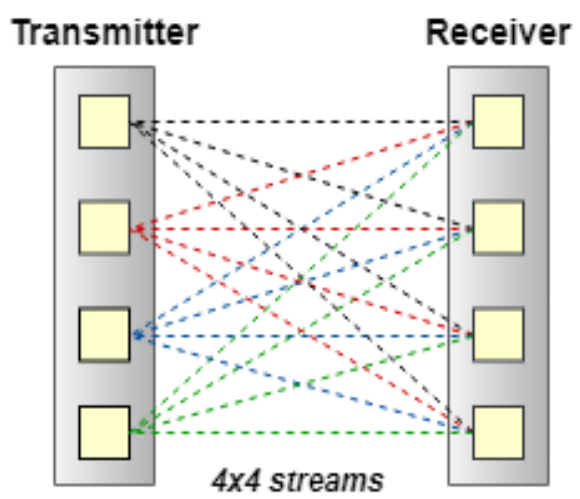

Figure 2 Signal Propagation (C) 2019 [1]

The obtained CSI packets from all links and subcarriers are indexed over time [33] (C) 2019 [1]. Let $l \in\{1, \cdots, L\}$ represent the antenna link, and $\operatorname{CSI}_{i l}(t)$ represent a complex number, describing the signal received at subcarrier $i \in\{1, \cdots, I\}$ at time $t$, defined by [33]:

$$
\operatorname{CSI}_{i l}(t)=\left|C S I_{i l}(t)\right| e^{\angle C S I_{i l}(t)}
$$

For a total of 16 streams with 58 subcarriers each, data samples consist of CSI measurements in the form of 3D matrices (C) 2019 [1]:

$$
\text { subcarrier indices } \times \text { packets } \times \text { streams }
$$

\subsection{Deployment of the Equipment}

The Aerial system was deployed in a $19 \times 35$ feet laboratory. The Wi-Fi devices (AP and STA) were placed diagonally across the laboratory. Initially, the two devices were both placed on the ground, at the corners of the laboratory, to perform 2D CSI measurements. Any captures collected under this setting are missing elevation information. Therefore, the AP was later mounted on the ceiling to perform 3D CSI measurements.

The Local Access Network (LAN) port of the provided AP device was connected to a port on the laboratory's router using an Ethernet cable. The AP device must always be plugged in and connected to the Internet, as it is responsible for reporting CSI captures to 
Aerial's cloud engine. On the other hand, the STA device just needs to be connected to a power outlet.

Aerial's Android application can be used to record data samples, and for live (realtime) demonstrations. An Application Programming Interface (API) is called by the application every 2 seconds to store data in Aerial's cloud. Aerial's app was installed on an Acer tablet, which was connected to the same network as the AP and STA. The app was configured by 1) pairing it to the AP device, and 2) signing up and creating a profile to associate with the AP device. Figure 3 below displays the deployment topology and the involved components. This system was used to acquire data for the thesis.

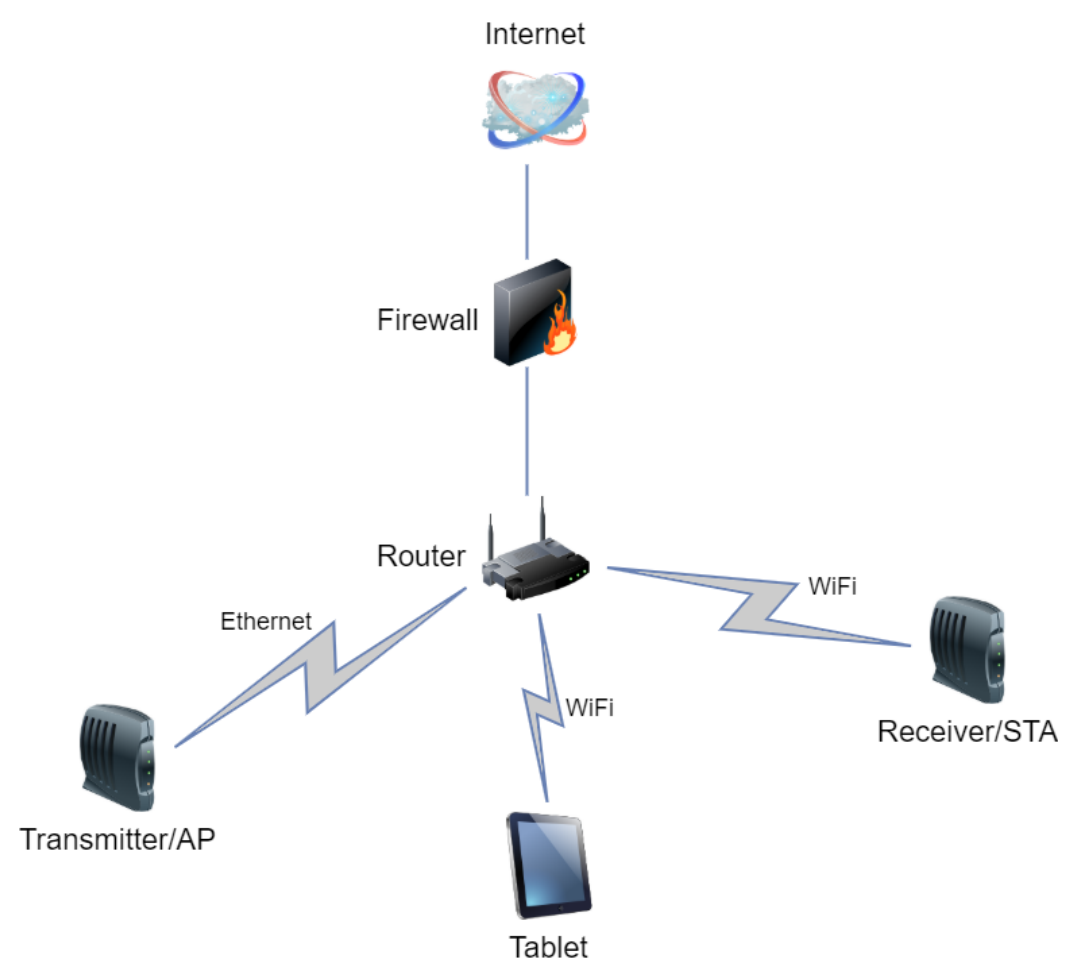

Figure 3 Deployment Topology and Components 


\section{Chapter 4: Proposed Solution}

Aerial's system was utilized to distinguish between two similar motions: the actions of sitting-down and standing-up from each other, and from the still (seated or standstill) case. The solution is composed of five main blocks: data collection and conversion, data preprocessing, feature extraction and scaling, class balancing, and classification. The data collection block is the stage at which sitting and standing experiments were performed to acquire the required Wi-Fi CSI signals for the study. The data preprocessing block is necessary to put the data in a proper format. Feature extraction is essential to determine attributes that identify an activity, as well as to reduce the dimensionality of the data. The class balancing block ensures that there is an equal distribution of data samples across all activity classes. Lastly, the classification block is split into two stages: training and testing. Sections 4.1 through 4.5 will discuss these five blocks, respectively. Figure 4 provides an overview of the developed system.

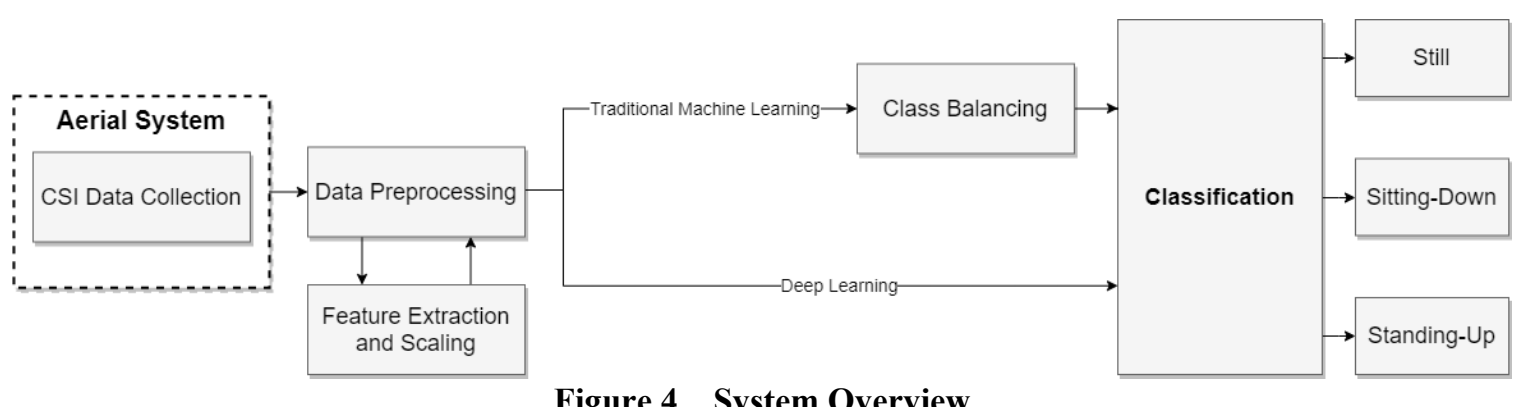

This chapter covers contributions 1 and 2. Contribution 1 focuses on performing data collection, preprocessing, visualization, and analysis on CSI captures; while contribution 2 focuses on applying a supervised machine learning technique to distinguish among sitting-down and standing-up actions. The following sections describe the proposed solution in more details. 


\subsection{Data Collection}

The first block in the system is data collection (see Figure 4). Test scenarios of differing sitting and standing speeds and styles, within various room arrangements, were conducted using the setup detailed in section 3.3 to address as many variations as could be tested (C) 2019 [1]. Various experimental setups were explored in terms of the position of Wi-Fi devices (AP and SAT/transmitter and receiver), and the location of activities relative to the devices. CSI data for a sequence of sitting-down and standing-up activities were acquired, as performed by a single subject, multiple times. Thus, four data sets were generated to represent data collected for:

1. a single activity location with both devices (i.e. AP and STA) on ground, as shown in Figure 11;

2. multiple activity locations, as shown in Figure 12, with both devices on ground;

3. multiple activity locations, as shown in Figure 12, with the AP at the ceiling and the STA on the ground; and

4. all cases combined (data sets 1,2 and 3).

Also, three volunteers were later asked to perform similar sitting and standing activities, at random room locations, with the AP mounted at the ceiling and the STA on the ground. Appendix A contains table summaries of the data collected and used for experimentation in this thesis.

The collected data consisted of 3D CSI captures, where each reading was a complex number of the form $\left|\operatorname{CSI}_{i l}(t)\right| e^{\angle C S I_{i l}(t)}$. Each CSI reading is collected by a specific 
subcarrier, at a specific timestamp. Each timestamp roughly corresponds to a second in time. With a sampling rate of 20 packets per second, about 1,800 packets of data were captured per sample, by 58 subcarriers, at 16 different streams. As such, the data consisted of $58 \times 16$ time series of CSI measurements. Figure 5 shows a sample of the magnitudes of a raw CSI capture, for one stream.

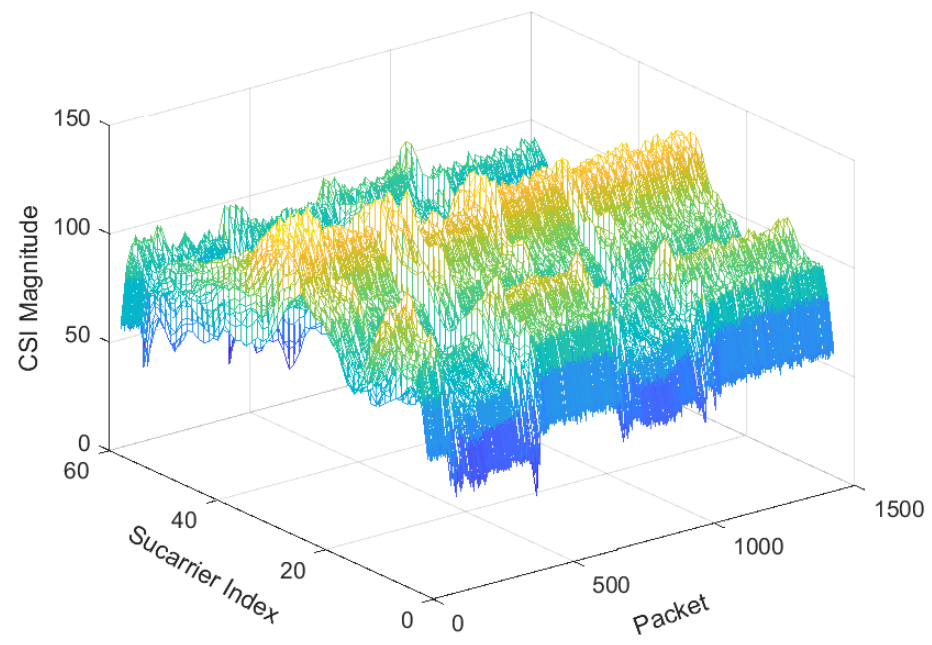

Figure 5 Raw CSI Capture

\subsection{Data Preprocessing}

The second block in the system is data preprocessing (see Figure 4). The acquired data/signals were preprocessed with filtering, dimensionality reduction, and segmentation (C) 2019 [1]. The signals were segmented and labelled as still, sitting-down, or standing-up. Then, a set of features was then extracted and scaled between 0 and 1 (see section 4.3). It was important to balance the classes in the data to improve the classification process (see section 4.4). Lastly, a series of traditional machine learning classifiers was used to distinguish among three classes of motion: stationary (seated or standing still), sittingdown, and standing-up (see section 4.5). The remainder of this section will further discuss the data preprocessing methodology. 
Since all streams reflect the same signal, one stream, rather than on all 16, was chosen to operate on $\mathbb{C} 2019$ [1]. As such, the best spatial stream (i.e. the one with the highest signal power and the lowest mean variance) was selected based on RSS and stability. The highest power represented the strongest signal, while the lowest long-term variance constituted a consistent, stable signal. This approach enabled us to operate on the stream that was mostly affected by the human activity.

The raw CSI measurements (complex numbers) in the selected stream were divided into their amplitudes, phases, and magnitudes (i.e. the square root of the sum of squared amplitude and phase components) (C) 2019 [1]. Then, a Hampel filter was applied to eliminate any outliers as suggested by [37], [38], and [45], along with a low-pass filter for noise removal. Since no human activity exceeds 2 hertz [33], the cut-off frequency of the low-pass filter was set to 2 hertz. Figure 6 displays a sample CSI capture after filtering.

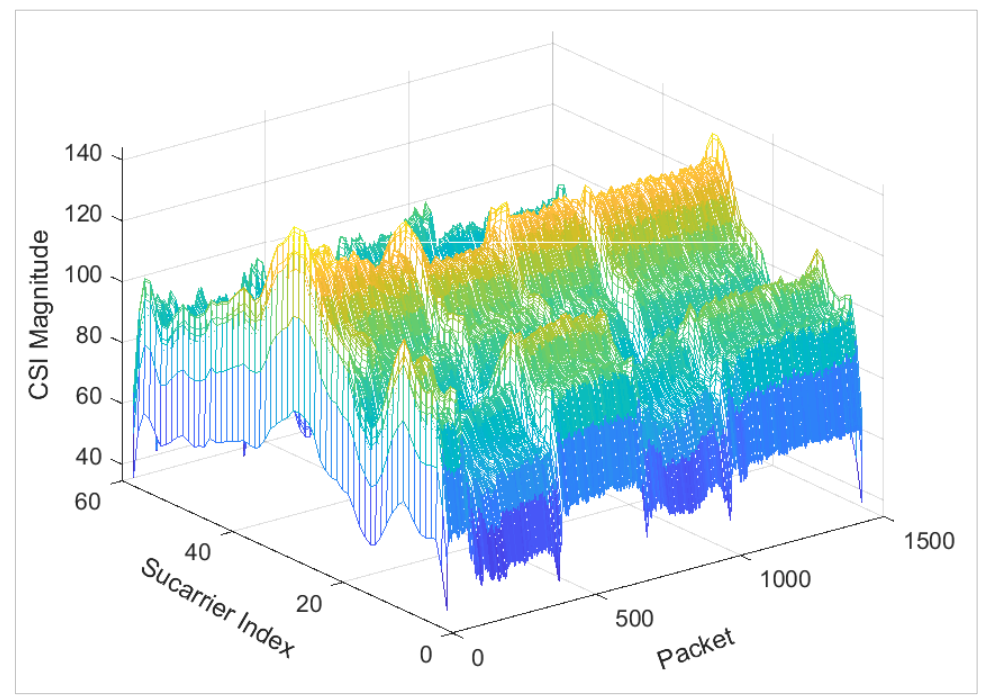

Figure 6 Filtered CSI Capture

Lastly, the CSI signals were segmented into 20-packet windows and accordingly labelled (C) 2019 [1]. A window length of 20 was chosen because some sitting-down and 
standing-up activities only take 1 second (i.e. about 20 packets) to complete. A timestamp was attached to each packet. To segment and label the data, the timestamps attached to the packets, as well as the event times recorded while performing sequences of sitting-down and standing-up activities, were utilized. This was achieved by finding the minimum absolute difference between an event's timestamp and the timestamps of the packets. Each event timestamp marked the beginning or the end of a stationary, sitting-down, or standingup activity. Once the appropriate starting timestamp/packet for an activity was located, the signal was segmented into 20-packet segments until the next event's timestamp was reached.

\subsection{Feature Extraction and Selection}

The third block in the system is feature extraction and scaling (see Figure 4). To reduce the dimensionality of the problem, the data was transformed from $3 \mathrm{D}$ to $2 \mathrm{D}$ by calculating metrics such as kurtosis, maximum, mean, minimum, skew, standard deviation, and variance along the subcarrier domain (C) 2019 [1]. The calculated attributes were chosen as they are commonly used across multiple state-of-the-art studies [32 - 47]. After this step, each data sample was represented as a time series of measured attributes. Figure 7 shows a sample plot of these time series in terms of features calculated on the magnitude of CSI measurements. One can observe that the time series seem to have some sort of a pattern associated with the movement from a sitting to a standing position, and vice versa (arrows in Figure 7). This pattern was evident on all of the attributes, which justified their selection for further analysis. Nonetheless, the moving variance of the mean as well as the moving variance of the variance, over a sliding window of 20 packets, were explored to more 
clearly visualize the patterns associated with the movements from a sitting to a standing position, and vice versa $\mathbb{C} 2019$ [1].

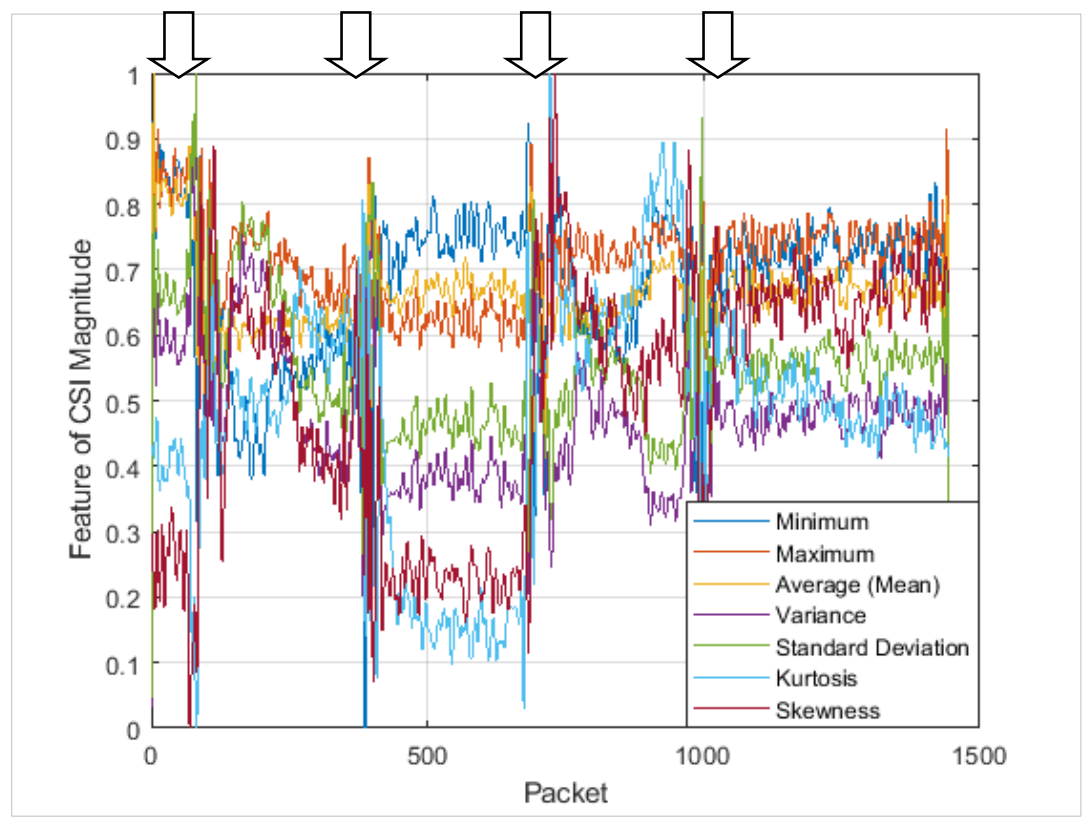

Figure 7 Sample Time Series of Subcarrier Features $\mathbb{C} 2019$ [1]

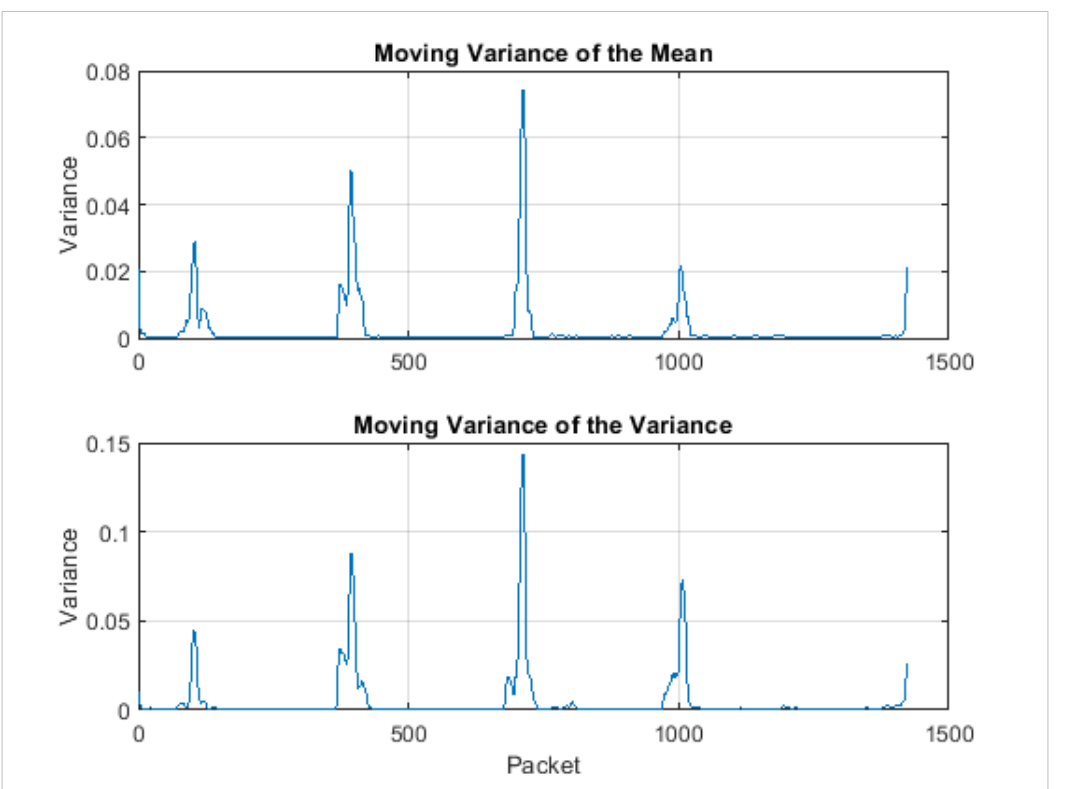

Figure 8 Sample Plots for Moving Variance of the Mean and of the Variance (c) 2019 [1]

Figure 8 represents a sample plot of the moving variance of the mean and the moving variance of the variance. Each peak is associated with a sitting-down or a standing-up 
activity, which shows a great potential to recognize human activities from the CSI of WiFi signals. Therefore, these peaks were used as another feature.

To deal with time series, the dimensionality of the data was further reduced by calculating metrics over the time (packet) domain (C) 2019 [1]. As a result, the final feature space, illustrated in Figure 9, was constructed at three levels:

1. Complex features, which consisted of $3 \mathrm{D}$ arrays of the amplitudes, phases, and magnitudes of raw CSI measurements (third dimension), obtained over time and sampled as packets (second dimension), by 58 subcarriers (first dimension), in the best spatial stream;

2. Subcarrier features, which consisted of $2 \mathrm{D}$ arrays of the kurtoses, maximums, means, minimums, skews, standard deviations, and variances of complex features (second dimension), calculated over the subcarrier domain (reduced dimension), for each packet (first dimension); and

3. Time features, which consisted of $1 \mathrm{D}$ points of the kurtosis, maximum, mean, minimum, peak, skew, standard deviation, and variance of subcarrier features, calculated across packets (reduced dimension).

As such, the feature space contained a total of 168 features per data sample. The 3D complex features were represented as three different matrices: CSI amplitudes, phases, and magnitudes, in terms of both subcarrier indices and packets of time. To reduce the subcarriers dimension, the $2 \mathrm{D}$ subcarrier features were calculated as 21 time series of kurtoses, maximums, means, minimums, skewnesses, standard deviations, and variances, for each of the 3D matrices in the set of complex features (i.e. CSI amplitudes, phases, and 
magnitudes). Finally, to reduce the packets dimension, the time features were determined for each packet, by calculating the kurtosis, maximum, mean, minimum, peak of moving variance of the mean, peak of moving variance of the variance, skewness, standard deviation, and variance of each of the 2D time series in the set of subcarrier features.

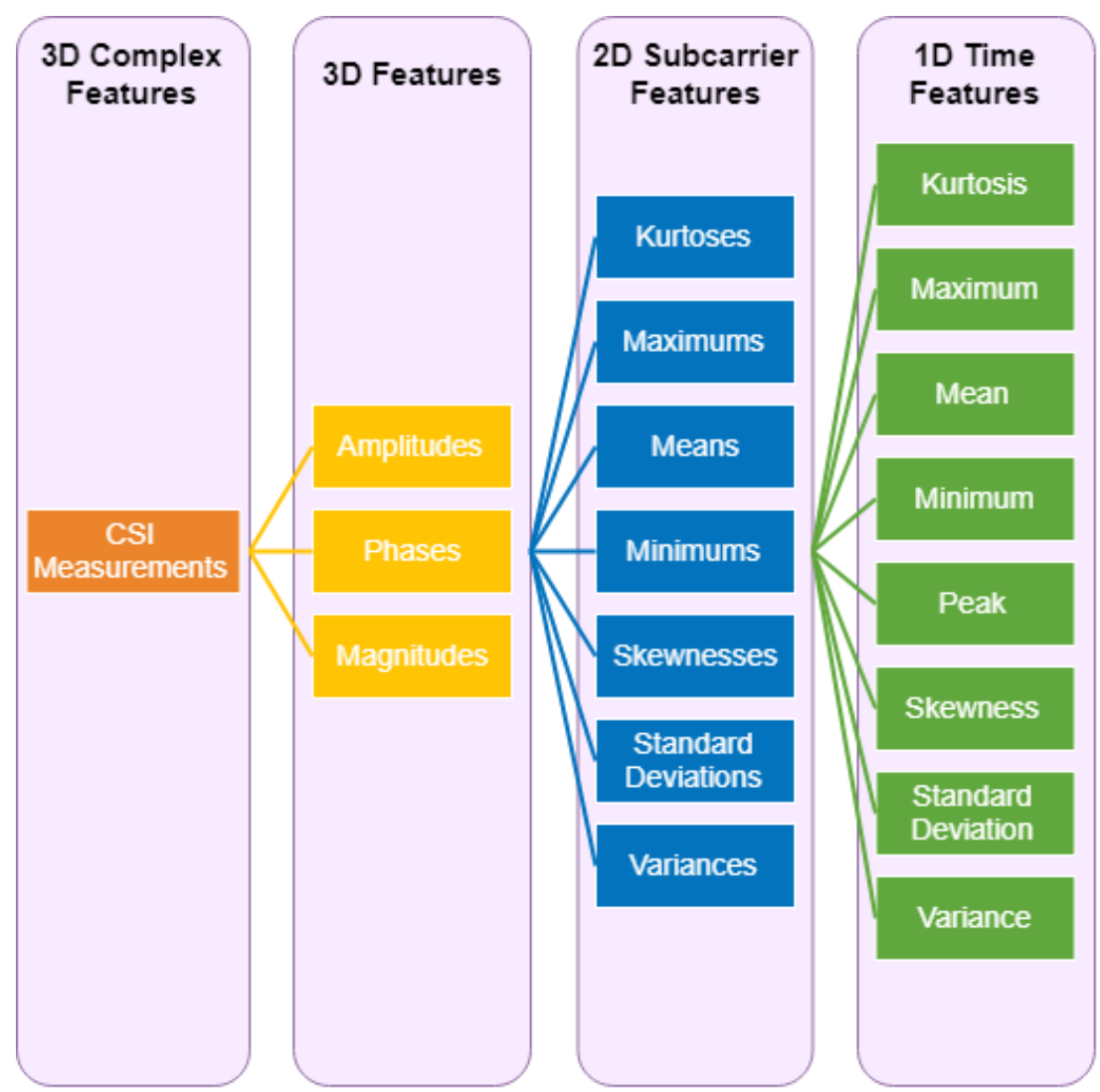

Figure 9 The Three Levels of Extracted Features

To bring all values into the range [ $\left[\begin{array}{l}1\end{array}\right]$, feature scaling was applied on these features via the following formula (C) 2019 [1]:

$$
x_{i}^{\prime}=\frac{x_{i}-X_{\min }}{X_{\max }-X_{\min }}
$$

where $X_{\min }$ and $X_{\max }$ are the minimum and maximum values of a feature vector. This approach is also known as min-max normalization. The range of all features should be normalized for them to contribute proportionately to the final decision of the classifiers. 
Feature scaling was tested by scaling the features space 1) over the subcarrier domain by normalizing the $2 \mathrm{D}$ subcarrier features, 2) across the time domain by normalizing the $1 \mathrm{D}$ time features, and 3) both ways (1 and 2). The third option improved the classification performance the most, as demonstrated using experimental results in section 5.2.3.

\subsection{Class Imbalance}

The fourth block in the system is class balancing (see Figure 4). Since the main interest was in the dynamics of the movements, lifting off the chair was counted as a "standing-up" activity, whereas getting on the chair was considered to be a "sitting-down" activity (C) 2019 [1]. To label the data, three labels (i.e. classes) were used: still (seated or standstill), sitting-down, and standing-up. Due to the nature of the employed data collection, the collected data contained more samples belonging to the "still" class, and, as such, the data set was imbalanced. To tackle this issue, the ADAptive SYNthetic Sampling (ADASYN) technique [52] was utilized to reduce class imbalance by synthesizing new samples of the minority class around the boundary between two classes instead of the interior of the minority class. This technique is an extension of the Synthetic Minority Oversampling TEchnique (SMOTE), which synthesizes new samples through a linear interpolation between existing minority class samples. In the context of this work, ADASYN was first applied to generate more samples of the "sitting-down" class as opposed to the "still" class. Then, it was applied to generate more samples of the "standingup" class as opposed to the "still" class. Afterwards, the synthesized "sitting-down" and "standing-up" samples were combined with the original data set. The used implementation of the ADASYN method takes six input parameters: a matrix of features, a vector of labels, the desired level of balance, the density of neighbors (for the k-nearest neighbors - KNN 
- call used in ADASYN [52]), the density for the KNN call used in SMOTE, and a Boolean that specifies if the features are normalized. The density parameters were set to 5 by default, while the normalization parameter was set to false. Setting the normalization parameter to false triggers unit variance normalization. The ADASYN method did not only assist in solving the class imbalance issue, but also provided more data to train the classifiers on.

\subsection{Classification}

The fifth block in the system is classification (see Figure 4). In terms of classification, both classical classifiers and deep learning classifiers were attempted. MATLAB's Classification Learner and Deep Network Designer apps were used to test a number of classification models. The following sections will discuss the classification methodologies in further details.

\subsubsection{Classification via Traditional Machine Learning}

MATLAB's Classification Learner was used to train different classifiers on the collected data (C) 2019 [1]. All available classifiers were leveraged to compare their

classification performances, with 5-fold cross validation, to prevent them from overfitting. The tested classifiers included decision trees, discriminant analysis, naïve Bayes, SVMs, KNN, and ensemble classifiers. The "No Free Lunch" theorem applies here, in the sense that there is no one classifier that works best in all circumstances. The performance of the classifiers is very dependent on the available data. However, the literature has shown use of decision tree [19, 20, 32], naïve Bayes [9, 20], SVM [9, 15, 20, 45, 46], and KNN [20] classifiers among others that were not utilized by the author of this thesis. 
For the decision trees, the fine, medium, and coarse versions were used. A fine decision tree has many leaves, and makes many fine distinctions between classes with a maximum number of 100 splits. A medium decision tree is a medium-complexity decision tree that has fewer leaves, and a maximum number of 20 splits, while a coarse decision tree has few leaves, and makes coarse distinctions between classes with a maximum number of 4 splits.

In terms of discriminant analysis, linear and quadratic discriminant classifiers with diagonal covariance regularization were tested. A linear discriminant is, in general, a faster and easier-to-interpret classifier, which creates linear boundaries between classes, while a quadratic discriminant classifier creates more complex (elliptical, parabolic, or hyperbolic) boundaries between classes, and, as such, has the potential to improve the classification.

To keep up with recent advances in research such as [9] and [20], naïve Bayes classifiers were added to the analysis (C) 2019 [1]. More specifically, two naïve Bayes approaches were tested: Gaussian and kernel. A Gaussian naïve Bayes classifier uses a Gaussian distribution for numeric predictors, while a kernel naïve Bayes classifier uses a kernel distribution, with a Gaussian kernel type and unbounded support. Both Gaussian and kernel naïve Bayes classifiers use a MultiVariate MultiNomial (MVMN) distribution for categorical predictors.

Among the SVMs, linear, quadratic, cubic, fine Gaussian, medium Gaussian, and coarse Gaussian SVMs were tested (C) 2019 [1]. The linear, quadratic, and cubic SVMs differ, as in the case of the discriminants above, in the complexity of the boundary that they create between classes. The fine, medium, and coarse Gaussian SVMs all use Gaussian kernels that differ in size. A fine Gaussian SVM enables finely-detailed distinctions 
between classes, using a Gaussian kernel with the kernel scale set to $\operatorname{sqrt}(P) / 4$, where $P$ is the number of predictors (i.e. features). A medium Gaussian SVM makes fewer distinctions between classes, using the Gaussian kernel with the kernel scale set to $\operatorname{sqrt}(P)$. Finally, a coarse Gaussian SVM makes coarse distinctions between classes, using the Gaussian kernel with the kernel scale set to $\operatorname{sqrt}(P) * 4$.

For the KNN classifiers, the fine, medium, coarse, cosine, cubic, and weighted versions were tested (C) 2019 [1]. The fine, medium, and coarse KNN classifiers make distinctions between classes using a Euclidean distance metric and equal distance weight, with the number of neighbours set to 1, 10, and 100, respectively. A cosine KNN classifier uses the cosine distance metric and equal distance weight, with the number of neighbours set to 10 , while a cubic KNN classifier uses the cubic distance metric, Minkowski, and equal distance weight, with the number of neighbours set to 10. A weighted KNN classifier uses a Euclidean distance metric and squared inverse distance weight, with the number of neighbours set to 10 .

Decision trees are constructed from conditional statements based on the boundaries in the variables of the inputted training data set, where each branch represents a condition and outputs a decision [48]. Discriminant analysis is the process of distinguishing between two or more classes using a boundary function. Naïve Bayes classification depends on a collection of probabilistic algorithms that are based on Bayes' theorem, which views each feature independently. SVMs attempt to identify the optimal hyperplane for categorizing data samples. An optimal hyperplane defines a clear gap between the data points in terms of space. The KNN algorithm determines the class of a sample according to its $k$ nearest neighbours. 
Finally, five different ensemble classifiers were also tried: boosted trees, bagged trees, subspace discriminant, subspace KNN, and RUSBoosted trees (C) 2019 [1]. The boosted trees model creates an ensemble of 30 medium decision trees using the AdaBoost algorithm. It uses relatively little memory or time, but might require more ensemble members than bagged trees. The bagged trees model is a bootstrap-aggregated ensemble of 30 fine decision trees. This type of model is often very accurate, but can be slow and memory-intensive for large data sets. The subspace models use the random subspace algorithm to create an ensemble of classifiers, with a subspace dimension of $P / 2$. The subspace discriminant model creates an ensemble of 30 discriminant classifiers, whereas the subspace KNN model creates an ensemble of $30 \mathrm{KNN}$ classifiers. They are good for many predictors (i.e. features), relatively fast for fitting and prediction, and low on memory usage. However, their performance can vary depending on the data. The RUSBoosted trees model is an extension of the boosted trees model. It is usually used when there is a skew in the data, with many more observations of one class (i.e. class imbalance (see section 4.4)). The next section will cover the proposed deep learning methodology.

\subsubsection{Classification via Deep Learning}

MATLAB's Deep Network Designer was used to design and implement a deep learning model. A Bidirectional Long Short-Term Memory (BiLSTM) neural network was designed to perform deep learning on the collected data. A BiLSTM network is a recurrent neural network, dedicated for learning long-term dependencies among the time steps of sequence data, and thus, is a good candidate for classifying the activities in the data set(s) of this work. Figure 10 displays the layers of the designed network. The network starts with 
a Sequence Input layer, and ends with a Fully Connected layer, a SoftMax layer, and a Classification Output layer to facilitate the prediction of class labels.

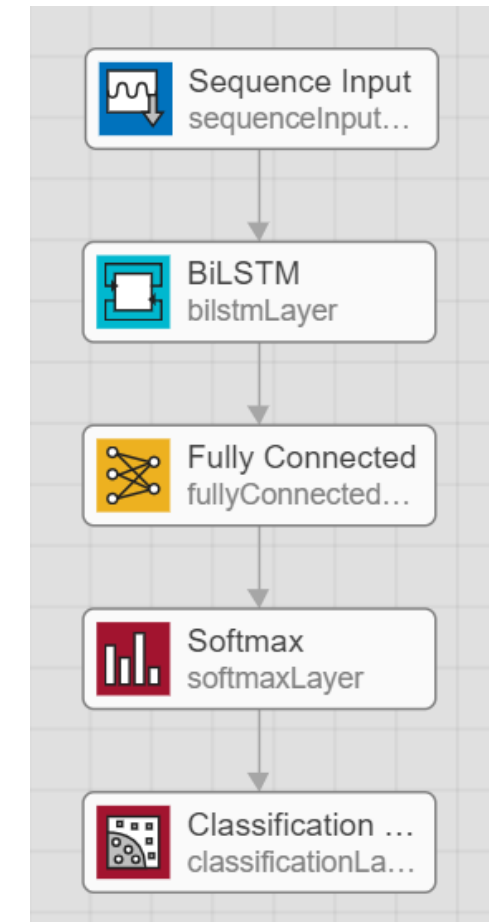

Figure 10 Layers of Designed Neural Network

The Sequence Input layer allows for the sequence of 21 features to be inputted to the network. Then, a BiLSTM layer of 128 hidden units/nodes, in default, is added to learn the bidirectional long-term dependencies between the time steps of the inputted time series (i.e. sequence data). These dependencies are useful for the network to learn from the complete time series at each time step. The number of hidden units/nodes specifies the amount of information that will be remembered between time steps. The Fully Connected layer multiplies the input by a weight matrix and then adds a bias vector, with an output size of 3 or 4 (number of activity classes). The SoftMax layer applies a softmax activation function to the input to determine the probabilities distribution of each activity class over all classes. Finally, the Classification Output layer computes the cross-entropy loss for 
multi-class classification with mutually exclusive classes. The cross-entropy loss is a measurement of performance.

Signals, such as the one shown in Figure 7, have been used to perform deep learning using the designed BiLSTM network. These signals represent the subcarrier features, which are time series of seven attributes: mean, minimum, maximum, variance, standard deviation, kurtosis, and skewness; calculated over the subcarrier domain of 3D matrices of CSI amplitudes, phases, and magnitudes. As such, the resulting feature space consists of 21 variables.

The ADAM (adaptive moment estimation) optimizer was then used to train the designed network. The network was set to train for a maximum of 100 epochs, where an epoch represents a full pass through the entire data set. Furthermore, the minimum batch size was set to 20 , meaning that the network is trained on 20 observations at each iteration of an epoch. The validation frequency was set to 50 iterations. 


\section{Chapter 5: Experimental Results}

This chapter will discuss the achieved experimental results for the contributions of this thesis.

\subsection{Distinguishing Sitting-down and Standing-up Activities}

A "preliminary" data set was initially collected to perform basic test cases for the proof of concept. This section includes details about the conducted experiments to collect the so-called "preliminary" data set, and results showing that the solution proposed in Chapter 4 can, in fact, distinguish between the dynamic motions of sitting-down and standing-up activities.

\subsubsection{Preliminary Sitting and Standing Experiments by One Subject}

Initially, data about Wi-Fi's CSI were collected by having one person (the author; a healthy female, 25 years old, 160 centimeters high, and 57 kilograms in weight) perform a sequence of sitting-down and standing-up activities at a single location in the room (C) 2019 [1]. The system was tested on a set of sitting-down and standing-up activities for a period of 90 seconds, as follows:

- $\quad$ sit for 5 seconds,

- $\quad$ stand for 15 seconds,

- $\quad$ sit for 15 seconds,

- $\quad$ stand for 15 seconds, and

- $\quad$ sit for the remaining time. 
The subject also carried a timer to record her event times as she sat down and stood up (C) 2019 [1]. This was done to keep track of the duration, as well as the start and end times of activities to help with data segmentation and labelling later on. At the start of a sequence of sitting-down and standing-up activities, the subject hit the timer's start button. Then, she waited for 5 seconds, hit the lap button, and started standing up. When she finished standing up, she hit the lap button again. She waited 15 seconds in a standstill position, hit the lap button, and started sitting down. Once fully seated, she hit the lap button again, and so on, until she completed the sequence. The lap times were recorded as a sequence of nine event times in minutes, seconds, and milliseconds, which corresponded to the duration of the laps (i.e. roughly between 1 to 15 seconds each). The actual timestamp of each event, within a sequence, was calculated by adding the preceding event times to its time. Event timestamps were then used to segment the data into separate samples, for each activity, as explained in section 4.2 above. Additionally, the activities were also videorecorded to get a ground truth of the subject's actions.

To allow the system to be able to identify a larger spectrum of sit-stand human activities, the following variations of actions were proposed for the training data, which was collected by the initial subject (C) 2019 [1]:

- sitting down and standing up in one step (i.e. no bending or leaning),

- sitting down and standing up in two steps by slightly bending forward then standing up or sitting down, and

- sitting down and standing up in three steps by leaning, bending, then standing up or sitting down. 
This was repeated multiple times, while facing a different direction (forward, backward, right, left, facing the transmitter/AP, facing the receiver/STA) each time (C) 2019 [1]. All of these experiments were completed with a chair placed in the middle of the laboratory, as in Figure 11.

Thus, a data set of CSI captures with the chair at the middle of the room, and both devices placed at ground-level (the so-called "preliminary" data set), was obtained. It was then filtered using both Hampel and low-pass filters, and segmented into 20-packet (1 second) segments. The so-called "preliminary" data set contained 2,835 samples. The number of samples within the so-called "preliminary" data set was then increased to 7,576 by generating new samples to balance the classes (see section 4.4). The so-called “preliminary" data set was used as a proof of concept data set to perform various tests.

\subsubsection{Preliminary Results}

The results for the preliminary case with the activity executed, by one individual, in the middle of the laboratory and both devices on the floor (as illustrated in Figure 11) are shown in Table 2, which summarizes the achieved classification accuracies. Note that the data used here was only filtered via both a Hampel and a low-pass filter, without applying any normalization or feature scaling yet.

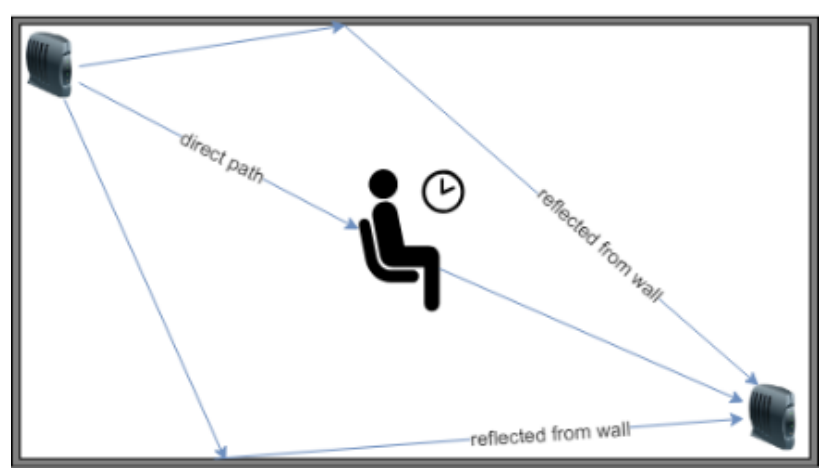

Figure 11 Initial activity location with the chair placed in the middle of the room $\mathbb{C} 2019$ [1] 
Table 2 Preliminary Classification Results

\begin{tabular}{|c|c|c|}
\hline Classification Method & Type & Accuracy $(\%)$ \\
\hline \multirow{3}{*}{ Decision Trees (DTs) } & Fine & 77.9 \\
\hline & Medium & 68.0 \\
\hline & Coarse & 60.3 \\
\hline \multirow{2}{*}{ Discriminant Analysis (DA) } & Linear & 57.1 \\
\hline & Quadratic & 53.2 \\
\hline \multirow{6}{*}{ Support Vector Machines (SVMs) } & Linear & 74.0 \\
\hline & Quadratic & 96.1 \\
\hline & Cubic & 97.2 \\
\hline & Fine Gaussian & 95.5 \\
\hline & Medium Gaussian & 97.5 \\
\hline & Coarse Gaussian & 70.5 \\
\hline \multirow{6}{*}{ K-Nearest Neighbours (KNN) } & Fine & 85.7 \\
\hline & Medium & 78.3 \\
\hline & Coarse & 60.2 \\
\hline & Cosine & 85.9 \\
\hline & Cubic & 77.0 \\
\hline & Weighted & 78.0 \\
\hline \multirow{5}{*}{ Ensemble Classifiers } & Boosted Trees & 76.2 \\
\hline & Bagged Trees & 96.1 \\
\hline & Subspace Discriminant & 73.7 \\
\hline & Subspace KNN & 75.0 \\
\hline & RUSBoosted Trees & 68.0 \\
\hline
\end{tabular}

In this case, the maximum accuracy, achieved by the medium Gaussian SVM, was 97.5\%. Accuracy is often not considered as a good estimate of classification performance, since it only measures the ratio of correct predictions, regardless of the amount of incorrect predictions. Therefore, confusion matrices will also be presented to offer a more complete view of the classification performance.

Table 3 illustrates the confusion matrix of the predictions of the medium Gaussian SVM for the so-called "preliminary" data set. As observed in the table, most samples were 
classified correctly. The green cells represent the number of correctly classified samples, while the pink cells represent the number of incorrectly classified samples. From 2,526 samples for the "still" class, 2,338 were correctly classified as still and 188 were wrongly classified as sitting-down or standing-up. For the "sitting-down" class, from 2,526 samples, 2,525 were correctly classified as sitting-down and one was wrongly classified as still. For the "standing-up" class, from 2,524 samples, 2,520 were correctly classified as standingup and 4 were wrongly classified.

Table 3 Confusion Matrix of the Medium Gaussian SVM for the "preliminary" Data Set

\begin{tabular}{|c|c|c|c|c|}
\hline \multirow{3}{*}{ 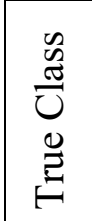 } & Still & 2338 & 85 & 103 \\
\hline & Sitting-down & 1 & 2525 & 0 \\
\hline & Standing-up & 3 & 1 & 2520 \\
\hline & & Still & Sitting-down & Standing-up \\
\hline & & \multicolumn{3}{|c|}{ Predicted Class } \\
\hline
\end{tabular}

These results demonstrate that it is possible to use Wi-Fi CSI to distinguish between the two similar motions of sitting-down and standing-up. Most of the utilized classifiers achieved high accuracies, and only 193 samples (out of 7,576) were misclassified by the best classifier.

\subsection{Pre-processing Techniques}

Based on the results in the previous section, additional preprocessing methods that could potentially improve the classification performance of the system were studied. As such, this section compares a number of preprocessing approaches to conclude regarding the best approach in terms of classification performance, which constitutes the third contribution of the thesis, from the list in section 1.4. 


\subsubsection{Experiments with Data Preprocessing Techniques}

To study the impact on the classification performance, multiple data preprocessing techniques were investigated. In particular, various normalization approaches were explored:

1. standardizing the raw CSI measurements over subcarriers for each packet/timestamp separately (or vice versa), using the z-score method:

$$
x_{i}^{\prime}=z_{i}=\frac{x_{i}-\mu}{\sigma}
$$

where $x_{i}$ is a sample CSI amplitude, phase, or magnitude; while $\mu$ and $\sigma$ are the mean and the standard deviation of a vector of CSI amplitudes, phases, or magnitudes, respectively. This approach normalized the data to have a mean of 0 and a standard deviation of 1 .

2. normalizing the raw CSI measurements over subcarriers for each packet/timestamp separately (or vice versa), using the following fixed-factor formula, determined by Aerial:

$$
\begin{aligned}
X^{\prime}= & \text { normalization factor } \cdot X \\
& \text { normalization factor } \\
& =\sqrt{(1 e 6 * 1.6508)\left(X \cdot X^{T}\right)}
\end{aligned}
$$

where $X$ is a vector of CSI amplitudes, phases, or magnitudes; and $X^{T}$ is the transpose of $X$. 
As can be seen in the following sections, the z-score standardization approach noticeably enhanced the performance over the other approaches.

\subsubsection{CSI Normalization}

As mentioned in section 5.2.1 above, multiple data preprocessing techniques were explored, beyond filtering. The explored techniques include normalizing the data using zscore standardization, or by a normalization factor. The data were normalized over the time domain and over the subcarrier domain. Normalizing the data over the subcarriers, using z-score standardization, had a better effect on the classification performance. Table 4 below compares the classification accuracies of the different classifiers, after applying the aforementioned normalization methods, on the so-called "preliminary" data set.

As observed in the table, standardization via the z-score method, across the subcarriers per timestamp significantly improved the classification performance over the other approaches. It achieved a maximum accuracy of $98.5 \%$ by the medium Gaussian SVM.

\subsubsection{Feature Scaling}

Feature scaling is essential to normalize the range of all features between 0 and 1 , for them to contribute proportionately to the prediction of the classifiers. Feature scaling was performed on the 2D subcarrier features (i.e. time series), the 1D time features (i.e. data points), and on both. Scaling the 2D subcarrier features as well as scaling all features, at both the 2D level and again at the 1D level, produced similar classification accuracies, as can be seen in Table 5. The first approach (scaling the 2D subcarrier features) achieved a maximum accuracy of $98.5 \%$ by the medium Gaussian SVM. 
Table 4 Comparison of Classification Accuracies achieved by Normalization Approaches

\begin{tabular}{|c|c|c|c|c|c|}
\hline \multirow{2}{*}{$\begin{array}{l}\text { Classification } \\
\text { Method }\end{array}$} & \multirow[b]{2}{*}{ Type } & \multicolumn{4}{|c|}{ Accuracy (\%) } \\
\hline & & $\begin{array}{l}\text { z-score } \\
\text { (time) }\end{array}$ & $\begin{array}{l}\text { factor } \\
\text { (time) }\end{array}$ & $\begin{array}{c}\text { Z-score } \\
\text { (subcarriers) }\end{array}$ & $\begin{array}{c}\text { factor } \\
\text { (subcarriers) }\end{array}$ \\
\hline \multirow{3}{*}{$\begin{array}{l}\text { Decision Trees } \\
\text { (DTs) }\end{array}$} & Fine & 77.1 & 77.3 & 75.7 & 76.7 \\
\hline & Medium & 68.4 & 69.5 & 66.8 & 66.8 \\
\hline & Coarse & 60.3 & 61.5 & 58.2 & 60.3 \\
\hline \multirow{2}{*}{$\begin{array}{l}\text { Discriminant } \\
\text { Analysis (DA) }\end{array}$} & Linear & 55.8 & 52.1 & 55.5 & 58.3 \\
\hline & Quadratic & 58.3 & 58.0 & 33.9 & 56.9 \\
\hline \multirow{6}{*}{$\begin{array}{c}\text { Support Vector } \\
\text { Machines (SVMs) }\end{array}$} & Linear & 76.0 & 74.4 & 76.7 & 73.0 \\
\hline & Quadratic & 96.2 & 95.8 & 96.5 & 95.9 \\
\hline & Cubic & 97.6 & 97.3 & 97.6 & 96.9 \\
\hline & $\begin{array}{c}\text { Fine } \\
\text { Gaussian }\end{array}$ & 92.3 & 92.6 & 92.0 & 92.8 \\
\hline & $\begin{array}{l}\text { Medium } \\
\text { Gaussian }\end{array}$ & 97.9 & 97.8 & 98.5 & 97.3 \\
\hline & $\begin{array}{c}\text { Coarse } \\
\text { Gaussian }\end{array}$ & 74.0 & 71.6 & 73.7 & 71.6 \\
\hline \multirow{6}{*}{$\begin{array}{c}\text { K-Nearest } \\
\text { Neighbours }(\mathrm{KNN})\end{array}$} & Fine & 85.5 & 84.7 & 81.7 & 85.4 \\
\hline & Medium & 77.9 & 76.4 & 73.4 & 77.6 \\
\hline & Coarse & 61.4 & 59.9 & 58.1 & 60.4 \\
\hline & Cosine & 87.9 & 85.1 & 83.5 & 86.1 \\
\hline & Cubic & 76.6 & 75.7 & 73.1 & 76.9 \\
\hline & Weighted & 77.8 & 76.5 & 73.2 & 77.4 \\
\hline \multirow{5}{*}{$\begin{array}{l}\text { Ensemble } \\
\text { Classifiers }\end{array}$} & $\begin{array}{l}\text { Boosted } \\
\text { Trees }\end{array}$ & 74.8 & 75.7 & 75.3 & 72.8 \\
\hline & $\begin{array}{l}\text { Bagged } \\
\text { Trees }\end{array}$ & 96.2 & 96.8 & 95.8 & 95.8 \\
\hline & $\begin{array}{c}\text { Subspace } \\
\text { Discriminant }\end{array}$ & 73.6 & 73.7 & 69.7 & 73.0 \\
\hline & $\begin{array}{c}\text { Subspace } \\
\text { KNN }\end{array}$ & 92.0 & 64.8 & 88.4 & 87.4 \\
\hline & $\begin{array}{c}\text { RUSBoosted } \\
\text { Trees }\end{array}$ & 69.4 & 70.1 & 69.0 & 67.4 \\
\hline
\end{tabular}


Table 5 Comparison of Classification Accuracies achieved by Feature Scaling Approaches

\begin{tabular}{|c|c|c|c|c|}
\hline \multirow{2}{*}{$\begin{array}{l}\text { Classification } \\
\text { Method }\end{array}$} & \multirow[b]{2}{*}{ Type } & \multicolumn{3}{|c|}{ Accuracy $(\%)$} \\
\hline & & $\begin{array}{c}\text { 2D Subcarrier } \\
\text { Features }\end{array}$ & $\begin{array}{l}\text { 1D Time } \\
\text { Features }\end{array}$ & $\begin{array}{c}\text { All } \\
\text { Features }\end{array}$ \\
\hline \multirow{3}{*}{$\begin{array}{c}\text { Decision Trees } \\
\text { (DTs) }\end{array}$} & Fine & 78.6 & 78.2 & 79.3 \\
\hline & Medium & 66.7 & 66.1 & 66.4 \\
\hline & Coarse & 60.2 & 58.7 & 59.7 \\
\hline \multirow{2}{*}{$\begin{array}{l}\text { Discriminant } \\
\text { Analysis (DA) }\end{array}$} & Linear & 53.7 & 57.3 & 53.4 \\
\hline & Quadratic & 60.1 & 53.3 & 60.3 \\
\hline \multirow{6}{*}{$\begin{array}{c}\text { Support Vector } \\
\text { Machines (SVMs) }\end{array}$} & Linear & 77.0 & 73.9 & 75.9 \\
\hline & Quadratic & 96.3 & 95.9 & 96.4 \\
\hline & Cubic & 97.9 & 97.1 & 97.7 \\
\hline & Fine Gaussian & 92.2 & 95.0 & 92.6 \\
\hline & Medium Gaussian & 98.5 & 97.4 & 98.4 \\
\hline & Coarse Gaussian & 75.4 & 71.0 & 75.6 \\
\hline \multirow{6}{*}{$\begin{array}{c}\text { K-Nearest } \\
\text { Neighbours }(\text { KNN) }\end{array}$} & Fine & 84.9 & 85.3 & 85.3 \\
\hline & Medium & 76.5 & 78.1 & 76.1 \\
\hline & Coarse & 60.3 & 60.7 & 60.1 \\
\hline & Cosine & 88.2 & 85.9 & 88.2 \\
\hline & Cubic & 75.0 & 77.2 & 75.0 \\
\hline & Weighted & 76.1 & 77.8 & 75.8 \\
\hline \multirow{5}{*}{$\begin{array}{l}\text { Ensemble } \\
\text { Classifiers }\end{array}$} & Boosted Trees & 77.8 & 75.5 & 77.8 \\
\hline & Bagged Trees & 96.1 & 96.1 & 96.1 \\
\hline & Subspace Discriminant & 76.7 & 73.7 & 75.3 \\
\hline & Subspace KNN & 88.3 & 90.2 & 90.9 \\
\hline & RUSBoosted Trees & 67.0 & 67.2 & 66.6 \\
\hline
\end{tabular}

\subsubsection{Optimization of Preprocessing Methodology}

By combining the best normalization and scaling approaches, the classification performance was optimized. Thus, a preprocessing methodology that included z-score standardization/normalization as an additional preprocessing step along with filtering, segmentation, and feature extraction and scaling was proven to perform better. 
Table 6 summarizes the classification accuracies after 1) standardizing the CSI data, over the subcarriers domain, through the z-score method, and 2) scaling subcarrier features or all features.

Table 6 Classification Results with Optimum Preprocessing Methodology

\begin{tabular}{|c|c|c|c|}
\hline \multirow{2}{*}{$\begin{array}{c}\text { Classification } \\
\text { Method }\end{array}$} & \multirow{2}{*}{ Type } & \multicolumn{2}{|c|}{ Accuracy $(\%)$} \\
\hline & & Subcarrier Features & All Features \\
\hline \multirow{3}{*}{$\begin{array}{c}\text { Decision Trees } \\
\text { (DTs) }\end{array}$} & Fine & 75.9 & 75.7 \\
\hline & Medium & 67.0 & 66.6 \\
\hline & Coarse & 58.3 & 58.8 \\
\hline \multirow{2}{*}{$\begin{array}{l}\text { Discriminant } \\
\text { Analysis (DA) }\end{array}$} & Linear & 56.6 & 56.5 \\
\hline & Quadratic & 65.1 & 66.1 \\
\hline \multirow{6}{*}{$\begin{array}{c}\text { Support Vector } \\
\text { Machines (SVMs) }\end{array}$} & Linear & 74.3 & 75.3 \\
\hline & Quadratic & 96.6 & 96.8 \\
\hline & Cubic & 97.7 & 97.8 \\
\hline & Fine Gaussian & 90.1 & 90.6 \\
\hline & Medium Gaussian & 98.8 & 98.8 \\
\hline & Coarse Gaussian & 74.7 & 74.7 \\
\hline \multirow{6}{*}{$\begin{array}{c}\text { K-Nearest } \\
\text { Neighbours } \\
\text { (KNN) }\end{array}$} & Fine & 82.3 & 82.2 \\
\hline & Medium & 73.3 & 73.2 \\
\hline & Coarse & 58.3 & 57.2 \\
\hline & Cosine & 86.9 & 86.8 \\
\hline & Cubic & 72.2 & 72.2 \\
\hline & Weighted & 73.1 & 72.8 \\
\hline \multirow{5}{*}{$\begin{array}{l}\text { Ensemble } \\
\text { Classifiers }\end{array}$} & Boosted Trees & 75.5 & 75.7 \\
\hline & Bagged Trees & 96.7 & 96.5 \\
\hline & Subspace Discriminant & 73.3 & 73.1 \\
\hline & Subspace KNN & 87.4 & 87.8 \\
\hline & RUSBoosted Trees & 69.2 & 68.7 \\
\hline
\end{tabular}

By comparing Tables 2, 4, 5 and 6, one can observe that applying a preprocessing methodology that includes both z-score standardization over the subcarriers and scaling all features improved the performance for most classifiers. For example, the maximum accuracy, achieved by the medium Gaussian SVM, went up to $98.8 \%$ for the so-called 
"preliminary" data set. Therefore, the results presented in the following sections are all presented for data that was preprocessed by filtering, z-score standardization, and by scaling all features.

Appendix B presents samples of the signals produced by applying the different preprocessing techniques.

\subsection{Subject Activity and Device Location Independence}

Once the first series of results demonstrated that the proposed solution distinguishes between still, sitting-down, and standing-up activity classes, different activity and device locations were also investigated. These are related to contributions 4 and 5 from the list in section 1.4. Contribution 4 focuses on the validation of the proposed solution for location and participant movement independence, while contribution 5 focuses on the impact of the location of Wi-Fi sensor devices on the classification. This section explains these contributions, by supporting them with additional experimental results.

\subsubsection{Experiments on Activity Location Independence}

As already briefly mentioned in section 4.1, the capability of the system to deal with different variations was explored. First, the location of the chair was changed, while keeping both the transmitter and the receiver on the ground. The chair was then positioned near the transmitter, near the receiver, and at the two empty corners of the room. All of the experiments were repeated with the transmitter mounted on the ceiling. Thus, test scenarios of differing sitting and standing speeds and styles were conducted, by one individual, within various room arrangements, to address as many variations as possible. Five experimental setups in terms of the position of Wi-Fi devices, and the location of activities 
relative to the devices were explored. Figure 12 shows top views of the various experimental setups.

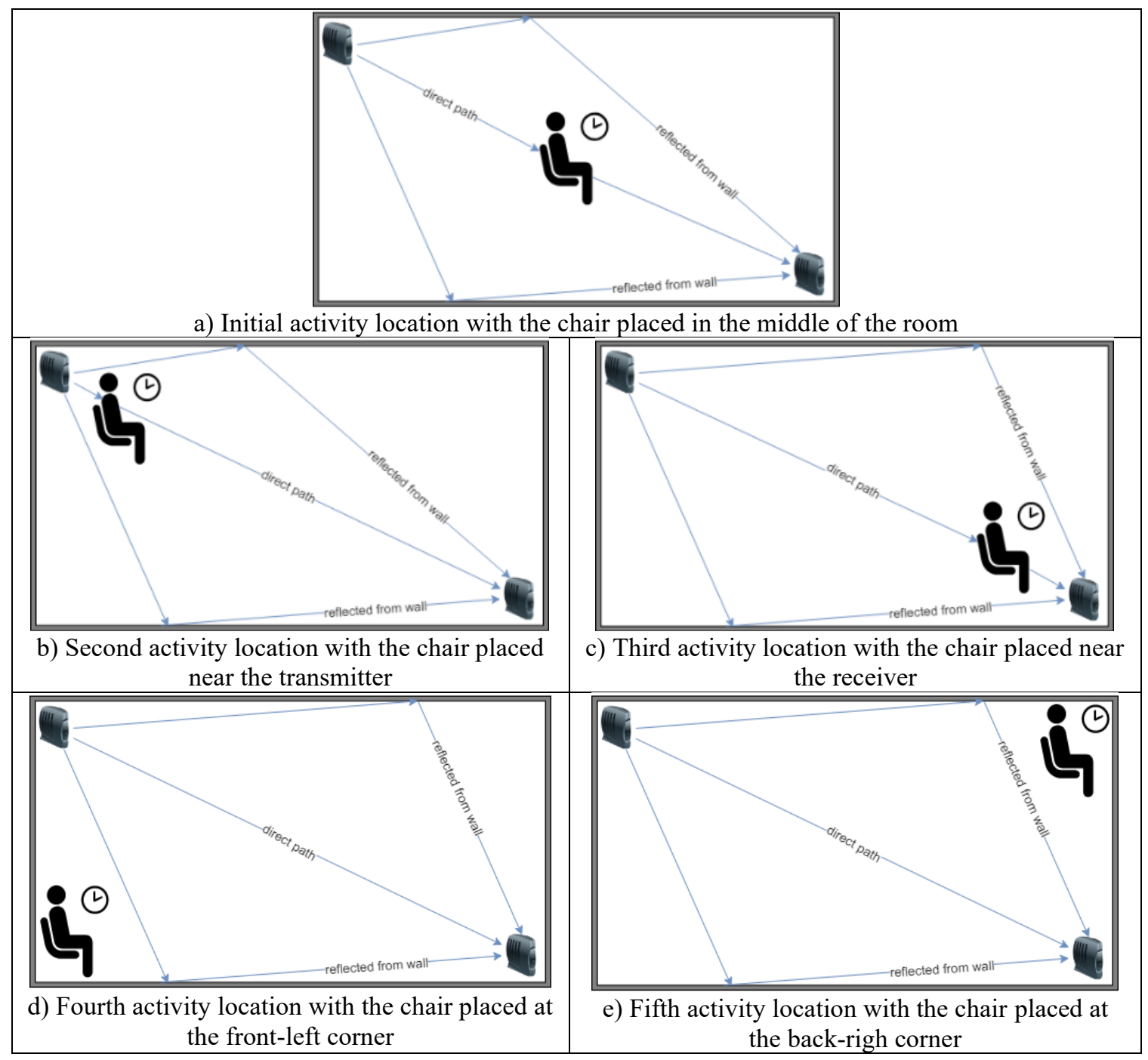

Figure 12 Top Views of Experimental Setups

In a real-life situation, a chair could be placed anywhere in a room. By changing the position of the chair around the laboratory, relative to the Wi-Fi devices, the system was enabled to identify more cases of sitting-down and standing-up activities. In turn, a data set was acquired for all scenarios and activity locations, with both devices placed at ground-level (the "floor" data set). Mounting one of the Wi-Fi devices at the ceiling of the 
laboratory added a third dimension to the CSI measurements. This helped to account for elevation information, such as the height of a person, in the sensed motions. Also, this enabled the sensing of Wi-Fi signals' propagation throughout the whole room, rather than propagating at the level of the ground only. Hence, this provided more coverage of the room in terms of both connectivity, and motion detection and identification. As a result, a data set of all scenarios and activity locations was acquired, with one device placed at ground-level and the other mounted at the ceiling (the "ceiling" data set). The so-called "preliminary", "floor", and "ceiling" data sets were then combined into one data set: the "floor + ceiling" data set. To summarize, four different data sets were acquired:

1. a preliminary data set with the chair at the middle of the room and both devices placed at ground-level (preliminary);

2. a data set of all scenarios and activity locations with both devices placed at ground-level (floor);

3. a data set of all scenarios and activity locations with one device placed at ground-level and the other mounted at the ceiling (ceiling); and

4. a full data set combining the "preliminary", the "floor", and the "ceiling" data sets (floor + ceiling).

Appendix $\mathrm{C}$ presents samples of the signals produced by performing the sitting and standing activities at different room locations. Appendix D presents samples of the signals produced by performing the sitting and standing activities under various device placements. The two appendices present how the pattern in CSI captures is influenced by activity location and device placement. 
After balancing the classes, the so-called "preliminary" data set contained 2,835 samples. The "floor" data set contained 6,849 samples, whereas the "ceiling" data set contained 5,208 samples. The "floor + ceiling" data set contained 12,057 samples. Note that the so-called "preliminary" data set is a subset of the "floor" data set. Table 7 summarizes the test scenarios. The second last row represents the size of the imbalanced data sets, before applying a class balancing mechanism (see section 4.4). The last row represents the size of the balanced data sets.

Table 7 Summary of Test Scenarios

\begin{tabular}{|c|c|c|c|c|}
\hline Data Set & Preliminary & Floor & Ceiling & Floor + Ceiling \\
\hline Transmitter/AP Position & \multicolumn{2}{|c|}{ floor } \\
\hline Receiver/STA Position & \multicolumn{2}{|c|}{ floor } & ceiling & ceiling or floor \\
\hline Chair/Activity Location & middle & $\begin{array}{r}\text { middle, near transmitter, near receiver, } \\
\text { front-left corner, back-right corner }\end{array}$ \\
\hline Body Orientation & front, back, left, right, towards transmitter, towards \\
\hline Number of Subjects & \multicolumn{3}{|r|}{ receiver } \\
\hline Number of CSI Captures & 42 & 102 & 78 & 180 \\
\hline Size of Original Data Set & 2,835 & 6,849 & 5,208 & 12,057 \\
\hline Size of Balanced Data Set & 7,569 & 18,156 & 13,488 & 31,637 \\
\hline
\end{tabular}

The classifiers were trained using the so-called "preliminary", "floor", "ceiling", and "floor + ceiling" data sets. They were tested using the built-in 5-fold cross validation in MATLAB's Classification Learner. Overall, the quadratic SVM, cubic SVM, the medium Gaussian SVM, and bagged trees outperformed the other classifiers. The effects 
on the classification performances by inputting the four data sets, one at a time, were studied. Comparing the four sets of results, the larger data sets ("floor", "ceiling", and "floor + ceiling") led to slightly downgraded classification performances from the so-called "preliminary" data set. Typically, this is what one would expect due to the wide range of variations in the data. However, it is important to note that the preliminary results are constrained to a very specific scenario. The following two sections will discuss the obtained classification results on the independence of classification performance from activity location and device placement, respectively.

\subsubsection{Independence of Activity Location}

Building on the preliminary results (see section 5.1), where the activity was executed in the center of the room, the subject performed actions in each of the four room corners, affecting their relative position to the transmitter and receiver. This was useful to analyze the independence of the system with regards to subjects' activity location. By collecting such data, the system was trained to recognize sitting and standing activities at different locations across the laboratory. To study the influence of training the classifiers on data collected by performing the experiments at different locations of the room, the results obtained from the so-called "preliminary" data set were compared with those obtained using the "floor" data set. As a reminder, the so-called "preliminary" data set was solely constituted of data from the experimental setup in Figure 12 (a), while the "floor" data set was constituted of data from all activity locations in Figure 12. Table 8 compares the classification accuracies.

In this case, the preliminary results demonstrated slightly better classification accuracies than the combined floor results, but only by approximately $3.4 \%$. This was 
expected, given that more variations of activities were involved in the "floor" data set. The slight decline in performance for the "floor" data set is because of the increase in data variations due to location independence. The naïve Bayes algorithm has also been added to the tests, Gaussian and kernel naïve Bayes in Table 9, to compare with state-of-the-art work, such as [9] and [20].

Table 8 Classification Results Demonstrating the Independence of Performance with Respect to Activity Location

\begin{tabular}{|c|c|c|c|c|}
\hline \multirow{2}{*}{ Classification Method } & \multirow{2}{*}{ Type } & \multicolumn{3}{|c|}{ Accuracy $(\%)$} \\
\hline & & Floor & Preliminary & Difference \\
\hline \multirow{3}{*}{ Decision Trees (DTs) } & Fine & 67.4 & 75.7 & 8.3 \\
\hline & Medium & 61.9 & 66.6 & 4.7 \\
\hline & Coarse & 56.9 & 58.8 & 1.9 \\
\hline \multirow{2}{*}{$\begin{array}{c}\text { Discriminant Analysis } \\
\text { (DA) }\end{array}$} & Linear & 54.6 & 56.5 & 1.9 \\
\hline & Quadratic & 60.9 & 66.1 & 5.2 \\
\hline \multirow{2}{*}{ Naïve Bayes (NB) } & Gaussian & 60.9 & 65.6 & 4.7 \\
\hline & Kernel & 72.3 & 79.8 & 7.5 \\
\hline \multirow{6}{*}{$\begin{array}{c}\text { Support Vector } \\
\text { Machines (SVMs) }\end{array}$} & Linear & 66.8 & 75.3 & 8.5 \\
\hline & Quadratic & 94.6 & 96.8 & 2.2 \\
\hline & Cubic & 97.6 & 97.8 & 0.2 \\
\hline & Fine Gaussian & 90.4 & 90.6 & 0.2 \\
\hline & Medium Gaussian & 98.5 & 98.8 & 0.3 \\
\hline & Coarse Gaussian & 70.9 & 74.7 & 3.8 \\
\hline \multirow{6}{*}{$\begin{array}{c}\text { K-Nearest Neighbours } \\
\text { (KNN) }\end{array}$} & Fine & 80.6 & 82.2 & 1.6 \\
\hline & Medium & 72.2 & 73.2 & 1 \\
\hline & Coarse & 57.2 & 57.2 & 0 \\
\hline & Cosine & 85.7 & 86.8 & 1.1 \\
\hline & Cubic & 71.4 & 72.2 & 0.8 \\
\hline & Weighted & 72.0 & 72.8 & 0.8 \\
\hline \multirow{5}{*}{ Ensemble Classifiers } & Boosted Trees & 65.8 & 75.7 & 9.9 \\
\hline & Bagged Trees & 95.9 & 96.5 & 0.6 \\
\hline & Subspace Discriminant & 64.5 & 73.1 & 8.6 \\
\hline & Subspace KNN & 86.5 & 87.8 & 1.3 \\
\hline & RUSBoosted Trees & 62.9 & 68.7 & 5.8 \\
\hline & & \multicolumn{2}{|c|}{ AVERAGE } & 3.4 \\
\hline
\end{tabular}




\subsubsection{Independence of Device Location}

Building on the preliminary results (see section 5.1), where the two Wi-Fi devices were both floor mounted, this part of the work evaluated the change in performance related to a floor-ceiling (3D) sensor placement. This included data from the various test scenarios and experimental setups (i.e. the "floor", "ceiling", and "floor + ceiling" data sets), as shown in Table 7 of section 5.3.1. Training the system to be able to classify CSI data of sitting and standing activities with the various sensor locations added more abstraction to its recognition capability. Table 9 compares the classification accuracies achieved on the "floor", "ceiling", and "floor + ceiling" data sets, whereas Figure 13 visualizes these results.

The medium Gaussian SVM achieved an accuracy of $98.8 \%$ for the so-called "preliminary" data set, $98.5 \%$ for the "floor" data set, $97.7 \%$ for the "ceiling" data set, and $97.8 \%$ for the "floor + ceiling" data set. Therefore, if the preliminary results were overlooked due to their associated limitations, the highest accuracy was achieved via the "floor" data set. This is likely because of the fact that placing both sensors on the ground, led to a smaller distance between the two devices, whereas a floor-ceiling placement did, in fact, place the sensors further apart.

Tables 10, 11, 12, and 13 illustrate the confusion matrices of the medium Gaussian SVM for the "preliminary", "floor", "ceiling", and "floor + ceiling" data sets, respectively. As observed in the tables, most samples were classified correctly. 
Table 9 Device Placement Classification Results

\begin{tabular}{|c|c|c|c|c|c|}
\hline \multirow{2}{*}{$\begin{array}{l}\text { Classification } \\
\text { Method }\end{array}$} & \multirow[b]{2}{*}{ Type } & \multicolumn{4}{|c|}{ Accuracy $(\%)$} \\
\hline & & Preliminary & Floor & Ceiling & $\begin{array}{l}\text { Floor + } \\
\text { Ceiling }\end{array}$ \\
\hline \multirow{3}{*}{$\begin{array}{c}\text { Decision Trees } \\
\text { (DTs) }\end{array}$} & Fine & 75.7 & 67.4 & 66.1 & 62.9 \\
\hline & Medium & 66.6 & 61.9 & 59.4 & 58.0 \\
\hline & Coarse & 58.8 & 56.9 & 54.6 & 55.6 \\
\hline \multirow{2}{*}{$\begin{array}{l}\text { Discriminant } \\
\text { Analysis (DA) }\end{array}$} & Linear & 56.5 & 54.6 & 54.2 & 52.6 \\
\hline & Quadratic & 66.1 & 60.9 & 60.9 & 58.1 \\
\hline \multirow{2}{*}{ Naïve Bayes (NB) } & Gaussian & 65.6 & 60.9 & 60.7 & 58.0 \\
\hline & Kernel & 79.8 & 72.3 & 71.7 & 69.0 \\
\hline \multirow{6}{*}{$\begin{array}{c}\text { Support Vector } \\
\text { Machines (SVMs) }\end{array}$} & Linear & 75.3 & 66.8 & 66.1 & 62.2 \\
\hline & Quadratic & 96.8 & 94.6 & 93.4 & 92.4 \\
\hline & Cubic & 97.8 & 97.6 & 96.6 & 97.1 \\
\hline & Fine Gaussian & 90.6 & 90.4 & 88.8 & 90.2 \\
\hline & Medium Gaussian & 98.8 & 98.5 & 97.7 & 97.8 \\
\hline & Coarse Gaussian & 74.7 & 70.9 & 69.1 & 67.1 \\
\hline \multirow{6}{*}{$\begin{array}{c}\text { K-Nearest } \\
\text { Neighbours } \\
(\mathrm{KNN})\end{array}$} & Fine & 82.2 & 80.6 & 79.9 & 79.7 \\
\hline & Medium & 73.2 & 72.2 & 71.1 & 71.3 \\
\hline & Coarse & 57.2 & 57.2 & 55.6 & 56.9 \\
\hline & Cosine & 86.8 & 85.7 & 84.1 & 85.5 \\
\hline & Cubic & 72.2 & 71.4 & 70.1 & 70.7 \\
\hline & Weighted & 72.8 & 72.0 & 71.5 & 71.2 \\
\hline \multirow{5}{*}{$\begin{array}{l}\text { Ensemble } \\
\text { Classifiers }\end{array}$} & Boosted Trees & 75.7 & 65.8 & 64.1 & 61.4 \\
\hline & Bagged Trees & 96.5 & 95.9 & 94.6 & 95.7 \\
\hline & $\begin{array}{c}\text { Subspace } \\
\text { Discriminant }\end{array}$ & 73.1 & 64.5 & 63.4 & 59.5 \\
\hline & Subspace KNN & 87.8 & 86.5 & 84.8 & 85.2 \\
\hline & RUSBoosted Trees & 68.7 & 62.9 & 61.5 & 58.8 \\
\hline
\end{tabular}




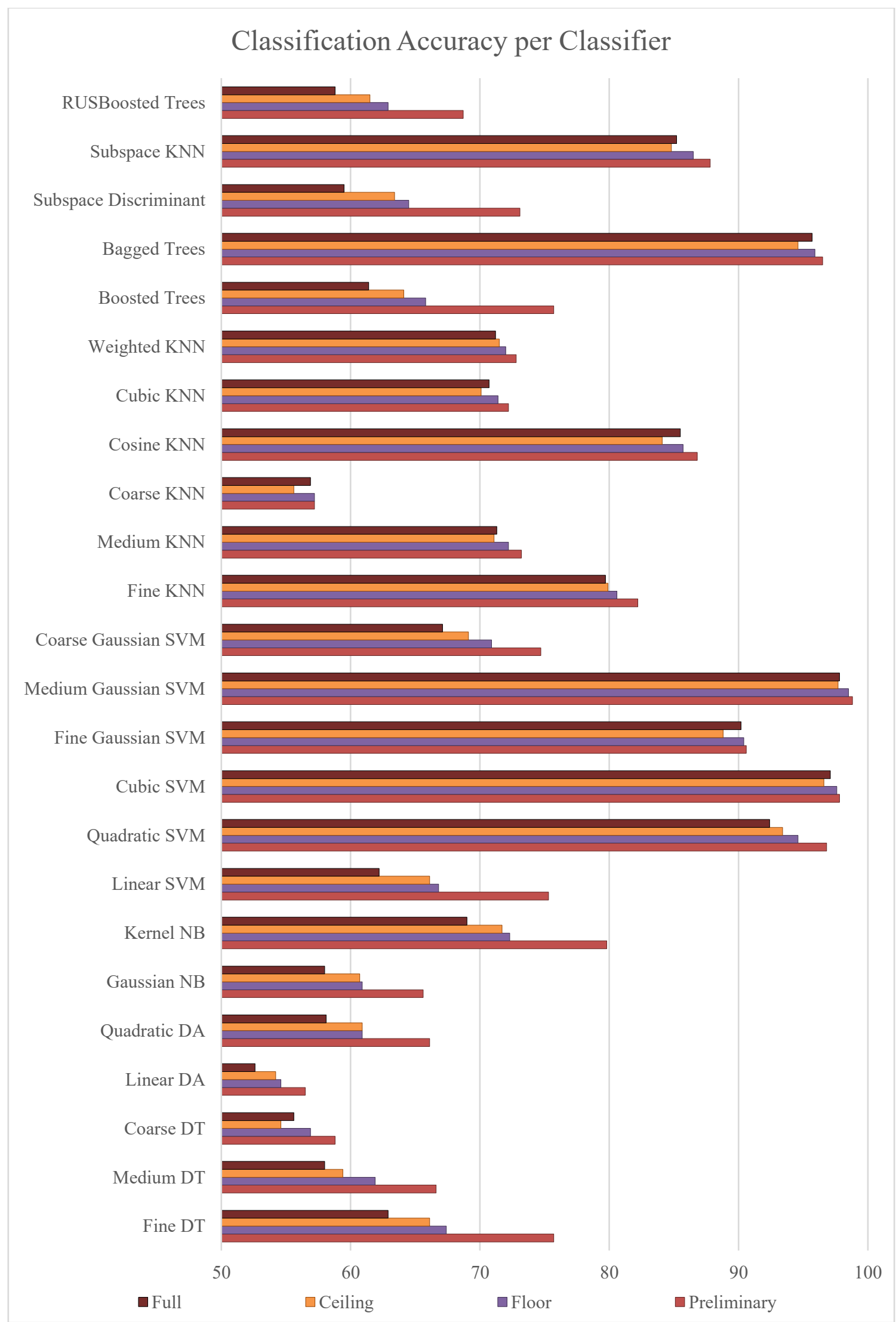

Figure 13 Classification Accuracy per Classifier for Device Placement 
According to Table 10, from 2,526 samples for the "still" class, 2,438 were correctly classified as still and 88 were wrongly classified as sitting-down or standing-up. For the "sitting-down" class, from 2,478 samples, 2,478 were correctly classified as sittingdown and 0 were wrongly classified. For the "standing-up" class, from 2,565 samples, 2,565 were correctly classified as standing-up and 0 were wrongly classified.

Table 10 Confusion Matrix of the Medium Gaussian SVM for the "preliminary" Data Set

\begin{tabular}{|c|c|c|c|c|}
\hline \multirow{2}{*}{$\underset{\Xi}{\Xi}$} & Still & 2438 & 31 & 57 \\
\cline { 2 - 5 } & Sitting-down & 0 & 2478 & 0 \\
\cline { 2 - 5 } & Standing-up & 0 & 0 & 2565 \\
\hline \multirow{2}{*}{} & Still & Sitting-down & Standing-up \\
\cline { 2 - 5 } & \multicolumn{3}{|c|}{ Predicted Class } \\
\cline { 2 - 5 }
\end{tabular}

Table 11 shows that from 6,054 samples for the "still" class, 5,789 were correctly classified as still and 265 were wrongly classified as sitting-down or standing-up. For the "sitting-down" class, from 5,993 samples, 5,979 were correctly classified as sitting-down and 14 were wrongly classified as still. For the "standing-up" class, from 6,109 samples, 6,108 were correctly classified as standing-up and one was wrongly classified as still.

Table 11 Confusion Matrix of the Medium Gaussian SVM for the "floor" Data Set

\begin{tabular}{|c|c|c|c|c|}
\hline \multirow{3}{*}{ 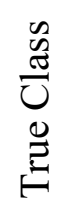 } & Still & 5789 & 106 & 159 \\
\hline & Sitting-down & 14 & 5979 & 0 \\
\hline & Standing-up & 1 & 0 & 6108 \\
\hline & & Still & Sitting-down & Standing-up \\
\hline & & \multicolumn{3}{|c|}{ Predicted Class } \\
\hline
\end{tabular}


As it can be observed in Table 12, from 4,480 samples for the "still" class, 4,194 were correctly classified as still and 286 were wrongly classified as sitting-down or standing-up. For the "sitting-down" class, from 4,456 samples, 4,442 were correctly classified as sitting-down and 14 were wrongly classified as still. For the "standing-up" class, from 4,552 samples, 4,547 were correctly classified as standing-up and 5 were wrongly classified as still.

Table 12 Confusion Matrix of the Medium Gaussian SVM for the "ceiling" Data Set

\begin{tabular}{|c|c|c|c|c|}
\hline \multirow{3}{*}{ 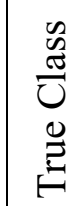 } & Still & 4194 & 128 & 158 \\
\hline & Sitting-down & 14 & 4442 & 0 \\
\hline & Standing-up & 5 & 0 & 4547 \\
\hline & & Still & Sitting-down & Standing-up \\
\hline & & \multicolumn{3}{|c|}{ Predicted Class } \\
\hline
\end{tabular}

Finally, according to Table 13, 9,894 from 10,534 samples of the "still" class, were correctly classified as still and 640 were wrongly classified as sitting-down or standing-up. In the case of the "sitting-down" class, from 10,654 samples, all but 32 were correctly classified as sitting-down. For the "standing-up" class, from 10,449 samples, all but 15 were correctly classified.

Table 13 Confusion Matrix of the Medium Gaussian SVM for the "floor + ceiling" Data Set

\begin{tabular}{|c|c|c|c|c|}
\hline \multirow{3}{*}{ 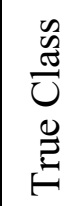 } & Still & 9894 & 275 & 365 \\
\hline & Sitting-down & 32 & 10622 & 0 \\
\hline & Standing-up & 15 & 0 & 10434 \\
\hline & & Still & Sitting-down & Standing-up \\
\hline & & \multicolumn{3}{|c|}{ Predicted Class } \\
\hline
\end{tabular}


The confusion matrices reflect the classification accuracies. Also, they show that most of the wrong predictions belonged to the "still" class, as they were classified as "sitting-down" or "standing-up". This might be due to the fact that the last position of a sitting-down activity is the seated/still position, whereas the last position of a standing-up activity is the standstill position. Nonetheless, the starting position of a sitting-down activity is the standstill position, whereas the starting position of a standing-up activity is the seated/still position.

\subsection{Time Repeatability and Drift}

The capability of the system to deal with time repeatability and signal drifting was tested by collecting the data on different days, at different times of the day. As such, this section details the experiments performed to complete contribution 6 .

\subsubsection{Experiments over Time}

Additional tests were performed to study the time independence and stability of the proposed solution. Two similar data sets of CSI captures were collected, 10 days apart, by having one subject perform a sequence of sitting-down and standing-up activities at the middle of the room. The data sets each corresponded to sitting-down and standing-up activities for a period of 90 seconds, as in section 5.1.1.

Each data set included the actions described in section 5.1.1, repeated six times, while facing different directions (forward, backward, right, left, facing the transmitter, facing the receiver).

To achieve this, the selected data sets were subsets of the so-called "preliminary" data set (see section 5.1). They were generated by dividing the so-called "preliminary" data 
set, which combined CSI data collected on two different days, for the same test scenarios, into two equal portions. Each proportion represented the data collected on one of the two days. The dates on which the data were collected were 10 days apart. These data sets were labelled as "day 1" and "day 2". Each of "day 1" and "day 2" data sets consisted of 18 CSI captures. After segmentation and balancing, the "day 1" data set included 3,284 samples, while the "day 2" data set contained 3,246.

Another subject collected two CSI captures that each include one sequence of sitting-down and standing-up activities, within the same day. This provided us with two data sets that were collected on the same day, within a shorter period of time. Therefore, testing the system, in terms of time repeatability and signal drift, using these data sets was considered as well. These data sets were labelled as "capture 1" and "capture 2". These

data sets were also processed through 1) Hampel and low-pass filtering, 2) z-score standardization/normalization, 3) data segmentation, 4) class labelling, 5) feature extraction and scaling, and 6) class balancing, as described in sections 4.2 through 4.4 and 5.2. After segmentation and balancing, the "capture 1" data set included 184 samples, while the "capture 2" data set contained 187.

\subsubsection{Time Repeatability and Independence}

The concept of time repeatability in Wi-Fi signals was addressed by collecting the data on different days of the week, at different times of the day; hence, enhancing the capability of the system to recognize activities at any point in time.

The classifiers were trained, and 5-fold cross validated on each of the "day 1" and “day 2 " data sets, individually. Table 14 compares the classification accuracies achieved 
by training on those data sets separately, along with the classification accuracies achieved by the combined "preliminary" data set. The overall average difference in classification accuracy between the preliminary results, and the results for the data from day 1 and 2 was approximately $4.8 \%$. This was because there were more data in the so-called "preliminary" data set. The overall difference in classification accuracy between the results for the data from day 1 and 2 was only $2.0 \%$ on average. Therefore, this showed that the proposed solution was not much affected by the concept of time repeatability, and performed relatively the same at different points in time.

Tables 15 and 16 illustrate the confusion matrices of the medium Gaussian SVM predictions for the data sets from day 1 and day 2, respectively. Approximately $99 \%$ of the samples were correctly classified by the medium Gaussian SVM. Only 16 samples of the data set for day 1 were misclassified, while 22 samples were misclassified from the data set for day 2 .

Specifically, for the "day 1" data set, Table 16 shows that from 1,085 samples for the "still" class, 1,070 were correctly classified as still and 15 were wrongly classified as sitting-down or standing-up. For the "sitting-down" class, all 1,109 samples were correctly classified as sitting-down. For the "standing-up" class, from 1,090 samples, 1,089 were correctly classified as standing-up and only one was wrongly classified as "still".

For the "day 2" data set, Table 16 shows that from 1,087 samples for the "still" class, 1,065 were correctly classified as still and 22 were wrongly classified as sitting-down or standing-up. For the "sitting-down" class, all 1,073 samples were correctly classified as 
sitting-down. For the "standing-up" class, all 1,086 samples were correctly classified as standing-up.

Table 14 Classification Results for Time Repeatability Evaluation

\begin{tabular}{|c|c|c|c|c|}
\hline \multirow{2}{*}{ Classification Method } & \multirow{2}{*}{ Type } & \multicolumn{3}{|c|}{ Accuracy (\%) } \\
\hline & & Preliminary & Day 1 & Day 2 \\
\hline \multirow{3}{*}{ Decision Trees (DTs) } & Fine & 75.7 & 84.1 & 87.4 \\
\hline & Medium & 66.6 & 72.7 & 78.0 \\
\hline & Coarse & 58.8 & 61.4 & 68.6 \\
\hline \multirow{2}{*}{$\begin{array}{c}\text { Discriminant Analysis } \\
\text { (DA) }\end{array}$} & Linear & 56.5 & 66.0 & 63.1 \\
\hline & Quadratic & 66.1 & 72.9 & 73.0 \\
\hline \multirow{2}{*}{ Naïve Bayes (NB) } & Gaussian & 65.6 & 72.9 & 73.0 \\
\hline & Kernel & 79.8 & 82.6 & 89.5 \\
\hline \multirow{6}{*}{$\begin{array}{c}\text { Support Vector } \\
\text { Machines (SVMs) }\end{array}$} & Linear & 75.3 & 84.6 & 87.0 \\
\hline & Quadratic & 96.8 & 97.4 & 97.6 \\
\hline & Cubic & 97.8 & 97.8 & 98.3 \\
\hline & Fine Gaussian & 90.6 & 91.3 & 90.6 \\
\hline & Medium Gaussian & 98.8 & 99.5 & 99.3 \\
\hline & Coarse Gaussian & 74.7 & 82.7 & 82.6 \\
\hline \multirow{6}{*}{$\begin{array}{c}\text { K-Nearest Neighbours } \\
\text { (KNN) }\end{array}$} & Fine & 82.2 & 83.0 & 84.0 \\
\hline & Medium & 73.2 & 74.0 & 75.1 \\
\hline & Coarse & 57.2 & 59.1 & 55.4 \\
\hline & Cosine & 86.8 & 87.3 & 88.4 \\
\hline & Cubic & 72.2 & 72.2 & 73.5 \\
\hline & Weighted & 72.8 & 73.7 & 74.8 \\
\hline \multirow{5}{*}{ Ensemble Classifiers } & Boosted Trees & 75.7 & 88.1 & 88.6 \\
\hline & Bagged Trees & 96.5 & 97.0 & 97.0 \\
\hline & Subspace Discriminant & 73.1 & 83.6 & 85.2 \\
\hline & Subspace KNN & 87.8 & 87.5 & 89.3 \\
\hline & RUSBoosted Trees & 68.7 & 73.7 & 78.9 \\
\hline
\end{tabular}


Table 15 Confusion Matrix Summarizing the Medium Gaussian SVM Predictions for "day 1"

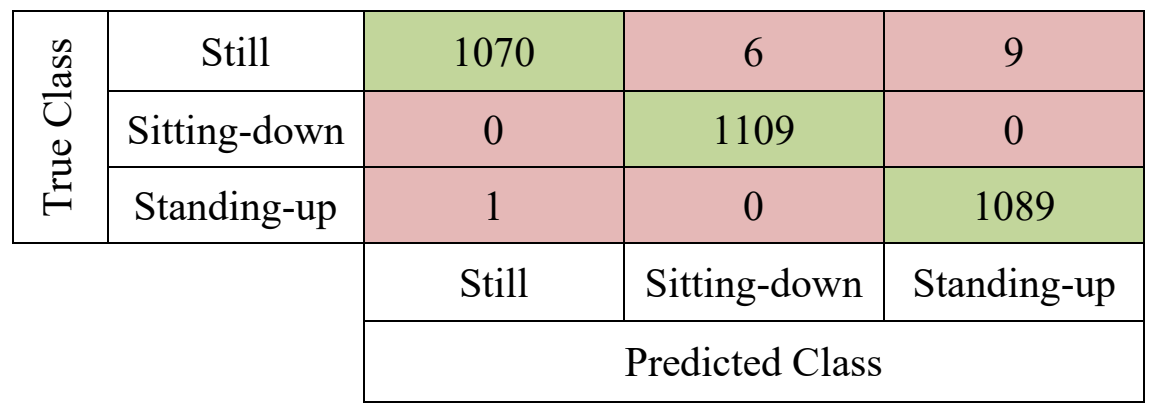

Table 16 Confusion Matrix Summarizing the Medium Gaussian SVM Predictions for "day 2"

\begin{tabular}{|c|c|c|c|c|}
\hline \multirow{3}{*}{ 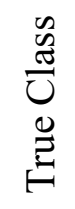 } & Still & 1065 & 9 & 13 \\
\hline & Sitting-down & 0 & 1073 & 0 \\
\hline & Standing-up & 0 & 0 & 1086 \\
\hline & & Still & Sitting-down & Standing-up \\
\hline & & \multicolumn{3}{|c|}{ Predicted Class } \\
\hline
\end{tabular}

Afterwards, the classifiers were trained, and 5-fold cross-validated on each of the "capture 1" and "capture 2" data sets, individually. Table 17 compares the classification accuracies achieved by training on those data sets separately, along with the classification accuracies achieved by the combined data set. The overall average difference in classification accuracy between the combined results, and the results for the data from captures 1 and 2 was approximately $4.3 \%$. This is likely because there were more data in the combined data set. The overall difference in classification accuracy between the results for the data from "capture 1" and "capture 2" was only $2.9 \%$ on average. Therefore, this showed that the proposed solution was not much affected by the concept of time repeatability, and performed relatively the same at different points in time. 
Table 17 Classification Results showing Time Repeatability on Two Data Sets from the Same Day

\begin{tabular}{|c|c|c|c|c|}
\hline \multirow{2}{*}{ Classification Method } & \multirow{2}{*}{ Type } & \multicolumn{3}{|c|}{ Accuracy $(\%)$} \\
\hline & & Capture 1 & Capture 2 & Combined \\
\hline \multirow{3}{*}{ Decision Trees (DTs) } & Fine & 92.9 & 97.3 & 96.2 \\
\hline & Medium & 92.9 & 97.3 & 96.2 \\
\hline & Coarse & 92.9 & 97.3 & 93.0 \\
\hline \multirow{2}{*}{$\begin{array}{l}\text { Discriminant } \\
\text { Analysis (DA) }\end{array}$} & Linear & 87.5 & 87.7 & 84.1 \\
\hline & Quadratic & 32.6 & 32.1 & 67.7 \\
\hline \multirow{2}{*}{ Naïve Bayes (NB) } & Gaussian & Failed & Failed & Failed \\
\hline & Kernel & 97.8 & 99.5 & 99.7 \\
\hline \multirow{6}{*}{$\begin{array}{c}\text { Support Vector } \\
\text { Machines (SVMs) }\end{array}$} & Linear & 97.8 & 95.7 & 96.2 \\
\hline & Quadratic & 98.4 & 96.3 & 97.6 \\
\hline & Cubic & 98.4 & 96.8 & 97.8 \\
\hline & Fine Gaussian & 96.2 & 98.9 & 94.6 \\
\hline & Medium Gaussian & 100 & 100 & 100 \\
\hline & Coarse Gaussian & 95.1 & 92.0 & 90.3 \\
\hline \multirow{6}{*}{$\begin{array}{c}\text { K-Nearest } \\
\text { Neighbours (KNN) }\end{array}$} & Fine & 83.7 & 80.7 & 79.8 \\
\hline & Medium & 74.5 & 67.9 & 72.0 \\
\hline & Coarse & 52.7 & 66.8 & 66.8 \\
\hline & Cosine & 94.0 & 89.3 & 85.2 \\
\hline & Cubic & 72.8 & 71.7 & 72.2 \\
\hline & Weighted & 73.9 & 67.9 & 72.0 \\
\hline \multirow{5}{*}{ Ensemble Classifiers } & Boosted Trees & 32.6 & 32.1 & 33.7 \\
\hline & Bagged Trees & 92.9 & 94.1 & 94.6 \\
\hline & Subspace Discriminant & 93.5 & 93.0 & 92.7 \\
\hline & Subspace KNN & 87.0 & 86.1 & 84.1 \\
\hline & RUSBoosted Trees & 32.6 & 32.1 & 46.6 \\
\hline
\end{tabular}

Tables 18, 19, and 20 demonstrate the confusion matrices of the predictions for capture 1, capture 2, and the combined data set, respectively. As the accuracy suggests, the confusion matrices also reflect perfect classification performances for the three data sets. 
Table 18 Confusion Matrix Summarizing the Medium Gaussian SVM Predictions for Capture 1

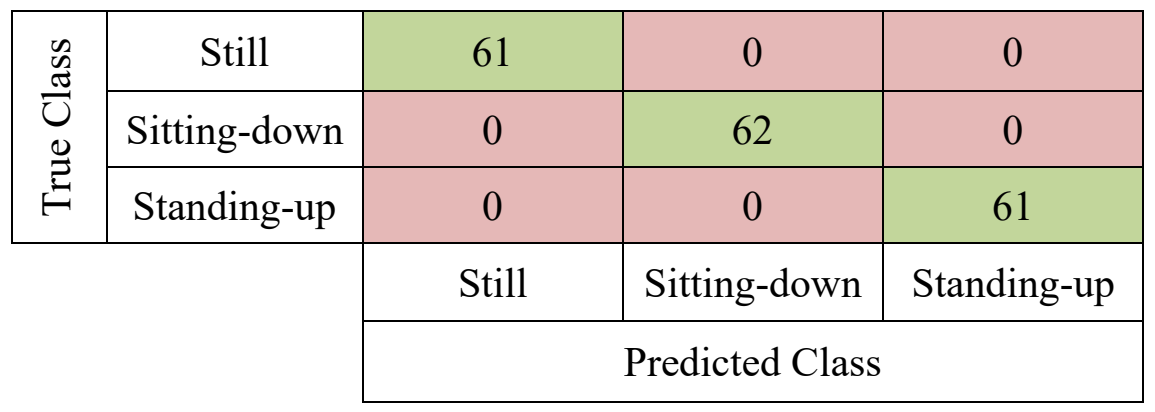

Table 19 Confusion Matrix Summarizing the Medium Gaussian SVM Predictions for Capture 2

\begin{tabular}{|c|c|c|c|c|}
\hline \multirow{2}{*}{$\underset{\Xi}{\tilde{\Xi}}$} & Still & 62 & 0 & 0 \\
\cline { 2 - 5 }$\underset{\Xi}{\Xi}$ & Sitting-down & 0 & 63 & 0 \\
\cline { 2 - 5 } & Standing-up & 0 & 0 & 62 \\
\hline \multirow{2}{*}{} & Still & Sitting-down & Standing-up \\
\cline { 2 - 5 } & \multicolumn{3}{|c|}{ Predicted Class } \\
\cline { 2 - 5 }
\end{tabular}

Table 20 Confusion Matrix Summarizing the Medium Gaussian SVM Predictions for the Data Set Combining Both Captures

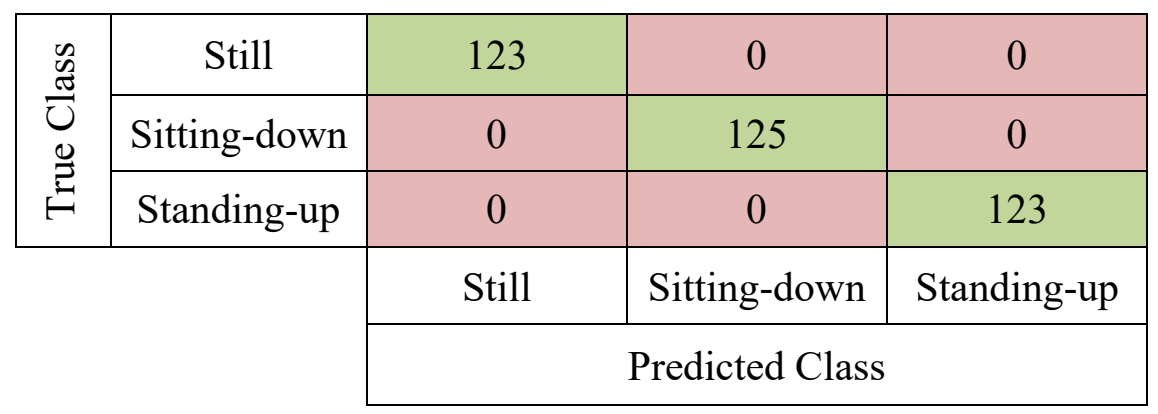




\subsubsection{Signal Drift}

Drift can be caused by many factors such as multipath interference, building attenuation, device and antenna orientation, dynamic changes in the environment, and signal noise [33]. Signal drift was addressed by using the data sets from "day 1" and "day 2 " as well as "capture 1" and "capture 2" above to evaluate the influence of signal drift on classification performance. The data from day 1 were first used to train the classifiers, and the data from day 2 were used to test them. Then, the process was repeated with day 2 as the training data set, and day 1 as the testing data set. Table 21 below summarizes the obtained results for "day 1 " and "day 2".

The results suggested that the proposed solution was negatively influenced by signal drift. The average difference between the training and testing classification accuracies, when day 1's data set was used for training and day 2's data set was used for testing, was approximately $34 \%$. In contrast, the average difference between the training and testing classification accuracies, when day 2's data set was used for training and day 1 's data set was used for testing, was approximately $36.9 \%$. So, the first scenario performed a little bit better than the second scenario, likely because the balanced training data set (using ADASYN) contained more samples in that specific case. The balanced data set for day 1 had 3,284 samples, while it contained 3,246 samples for day 2. 
Table 21 Classification Results for Signal Drift Evaluation

\begin{tabular}{|c|c|c|c|c|c|}
\hline \multirow{2}{*}{$\begin{array}{l}\text { Classification } \\
\text { Method }\end{array}$} & \multirow[b]{2}{*}{ Type } & \multicolumn{4}{|c|}{ Accuracy $(\%)$} \\
\hline & & $\begin{array}{c}\text { Training } \\
\text { on "Day 1" }\end{array}$ & $\begin{array}{c}\text { Testing on } \\
\text { "Day 2" }\end{array}$ & $\begin{array}{c}\text { Training } \\
\text { on "Day 2" }\end{array}$ & $\begin{array}{l}\text { Testing on } \\
\text { "Day 1" }\end{array}$ \\
\hline \multirow{3}{*}{$\begin{array}{c}\text { Decision } \\
\text { Trees (DTs) }\end{array}$} & Fine & 84.0 & 43.9 & 85.8 & 45.0 \\
\hline & Medium & 73.8 & 45.5 & 76.3 & 51.3 \\
\hline & Coarse & 61.6 & 46.0 & 68.8 & 50.9 \\
\hline \multirow{2}{*}{$\begin{array}{c}\text { Discriminant } \\
\text { Analysis } \\
\text { (DA) }\end{array}$} & Linear & 65.6 & 51.0 & 63.6 & 47.0 \\
\hline & Quadratic & 73.2 & 51.6 & 72.1 & 48.8 \\
\hline \multirow{2}{*}{$\begin{array}{c}\text { Naïve Bayes } \\
\text { (NB) }\end{array}$} & Gaussian & 73.2 & 51.6 & 72.1 & 48.8 \\
\hline & Kernel & 82.3 & 56.5 & 88.2 & 48.6 \\
\hline \multirow{6}{*}{$\begin{array}{l}\text { Support } \\
\text { Vector } \\
\text { Machines } \\
\text { (SVMs) }\end{array}$} & Linear & 84.4 & 49.0 & 87.1 & 45.9 \\
\hline & Quadratic & 97.0 & 49.0 & 97.8 & 41.4 \\
\hline & Cubic & 97.7 & 48.5 & 98.2 & 41.6 \\
\hline & Fine Gaussian & 91.1 & 33.5 & 90.9 & 33.0 \\
\hline & $\begin{array}{l}\text { Medium } \\
\text { Gaussian }\end{array}$ & 99.5 & 45.3 & 99.3 & 39.4 \\
\hline & $\begin{array}{c}\text { Coarse } \\
\text { Gaussian }\end{array}$ & 82.6 & 55.3 & 82.6 & 50.8 \\
\hline \multirow{6}{*}{$\begin{array}{c}\text { K-Nearest } \\
\text { Neighbours } \\
(\text { KNN) }\end{array}$} & Fine & 83.1 & 44.5 & 83.9 & 45.5 \\
\hline & Medium & 73.8 & 40.4 & 74.9 & 42.8 \\
\hline & Coarse & 58.6 & 38.9 & 53.7 & 36.5 \\
\hline & Cosine & 87.4 & 41.9 & 89.0 & 41.0 \\
\hline & Cubic & 72.4 & 40.1 & 73.5 & 42.3 \\
\hline & Weighted & 73.7 & 40.7 & 74.8 & 44.4 \\
\hline \multirow{5}{*}{$\begin{array}{l}\text { Ensemble } \\
\text { Classifiers }\end{array}$} & $\begin{array}{c}\text { Boosted } \\
\text { Trees }\end{array}$ & 87.3 & 57.2 & 87.9 & 47.5 \\
\hline & Bagged Trees & 97.5 & 49.5 & 97.2 & 44.6 \\
\hline & $\begin{array}{c}\text { Subspace } \\
\text { Discriminant }\end{array}$ & 83.6 & 51.1 & 85.9 & 45.8 \\
\hline & $\begin{array}{c}\text { Subspace } \\
\text { KNN }\end{array}$ & 87.6 & 46.6 & 89.1 & 48.9 \\
\hline & $\begin{array}{c}\text { RUSBoosted } \\
\text { Trees }\end{array}$ & 75.9 & 53.6 & 76.9 & 51.3 \\
\hline \multicolumn{2}{|c|}{ AVERAGE DIFFERENCE } & \multicolumn{2}{|c|}{34.0} & \multicolumn{2}{|c|}{36.9} \\
\hline
\end{tabular}


Table 22 below summarizes the classification results obtained by training the classifiers on "capture 1" and testing on "capture 2", which were collected on the same day, within a very short period of time apart. The average difference between the training and testing classification accuracies, when capture 1's data set was used for training and capture 2's data set was used for testing, was approximately $34.7 \%$. In contrast, the average difference between the training and testing classification accuracies, when capture 2's data set was used for training and capture 1's data set was used for testing, was approximately $40.6 \%$. So, the first scenario performed better than the second scenario.

The results suggested that the proposed solution was badly affected by signal drift, even on the same day. This showed that time was not the main factor behind performance changes, as the performance degraded even when using two data sets that were captured within a very short amount of time from one another. One can also question the integrity of the data collected by the volunteer as there is no guarantee that he/she performed the exercise as expected.

The poor results suggest that the system will need to adopt an ongoing training mechanism (i.e. adaptive learning), as it was unable to recognize the samples from CSI captures that it was not trained on. This was apparent since there was a significant drop in training and testing performances, although the training and testing data sets included similar data. Furthermore, the best classifier, as indicated by the training performance, may not necessarily be the best according to the testing performance. For example, the SVMs tended to achieve the highest classification accuracies in training, but they did not hold up when tested on other data sets. The KNN classifiers, however, seemed to hold up better as their performances were relatively consistent through both the training and testing stages. 
Table 22 Classification Results showing Signal Drift on Two Data Sets from the Same Day

\begin{tabular}{|c|c|c|c|c|c|}
\hline \multirow[b]{2}{*}{$\begin{array}{l}\text { Classification } \\
\text { Method }\end{array}$} & \multirow[b]{2}{*}{ Type } & \multicolumn{4}{|c|}{ Accuracy $(\%)$} \\
\hline & & $\begin{array}{l}\text { Training } \\
\text { on } \\
\text { Capture } 1\end{array}$ & $\begin{array}{c}\text { Testing } \\
\text { on } \\
\text { Capture } 2\end{array}$ & $\begin{array}{l}\text { Training } \\
\text { on } \\
\text { Capture } 2\end{array}$ & $\begin{array}{c}\text { Testing } \\
\text { on } \\
\text { Capture 1 }\end{array}$ \\
\hline \multirow{3}{*}{$\begin{array}{c}\text { Decision Trees } \\
\text { (DTs) }\end{array}$} & Fine & 92.4 & 54.0 & 98.4 & 30.4 \\
\hline & Medium & 92.4 & 54.0 & 98.4 & 30.4 \\
\hline & Coarse & 92.4 & 54.6 & 98.4 & 30.4 \\
\hline \multirow{2}{*}{$\begin{array}{l}\text { Discriminant } \\
\text { Analysis (DA) }\end{array}$} & Linear & 87.0 & 46.5 & 88.8 & 47.3 \\
\hline & Quadratic & 32.6 & 33.7 & 32.1 & 33.7 \\
\hline \multirow{2}{*}{$\begin{array}{l}\text { Naïve Bayes } \\
\text { (NB) }\end{array}$} & Gaussian & Failed & Failed & Failed & Failed \\
\hline & Kernel & 98.9 & 31.0 & 100 & 33.15 \\
\hline \multirow{6}{*}{$\begin{array}{l}\text { Support Vector } \\
\text { Machines } \\
\text { (SVMs) }\end{array}$} & Linear & 98.9 & 32.1 & 94.7 & 32.1 \\
\hline & Quadratic & 98.4 & 32.1 & 97.3 & 32.1 \\
\hline & Cubic & 98.4 & 32.1 & 97.9 & 32.1 \\
\hline & Fine Gaussian & 96.7 & 33.2 & 99.5 & 33.2 \\
\hline & $\begin{array}{l}\text { Medium } \\
\text { Gaussian }\end{array}$ & 100 & 33.2 & 100 & 33.2 \\
\hline & $\begin{array}{c}\text { Coarse } \\
\text { Gaussian }\end{array}$ & 95.1 & 31.6 & 93.0 & 41.3 \\
\hline \multirow{6}{*}{$\begin{array}{c}\text { K-Nearest } \\
\text { Neighbours } \\
(\text { KNN) }\end{array}$} & Fine & 83.7 & 74.9 & 80.7 & 53.8 \\
\hline & Medium & 71.2 & 65.8 & 67.4 & 58.2 \\
\hline & Coarse & 51.6 & 33.7 & 66.8 & 63.6 \\
\hline & Cosine & 90.8 & 64.2 & 89.8 & 46.7 \\
\hline & Cubic & 71.2 & 64.2 & 71.1 & 57.6 \\
\hline & Weighted & 70.7 & 67.9 & 67.4 & 59.8 \\
\hline \multirow{5}{*}{$\begin{array}{l}\text { Ensemble } \\
\text { Classifiers }\end{array}$} & Boosted Trees & 32.6 & 33.7 & 32.1 & 33.7 \\
\hline & Bagged Trees & 96.2 & 41.7 & 97.3 & 51.1 \\
\hline & $\begin{array}{c}\text { Subspace } \\
\text { Discriminant }\end{array}$ & 92.9 & 50.3 & 87.2 & 40.2 \\
\hline & $\begin{array}{c}\text { Subspace } \\
\text { KNN }\end{array}$ & 89.1 & 75.9 & 86.1 & 45.7 \\
\hline & $\begin{array}{l}\text { RUSBoosted } \\
\text { Trees }\end{array}$ & 32.6 & 33.7 & 32.1 & 33.7 \\
\hline \multicolumn{2}{|c|}{ AVERAGE DIFFERENCE } & \multicolumn{2}{|c|}{34.7} & \multicolumn{2}{|c|}{40.6} \\
\hline
\end{tabular}




\subsection{Testing and Validation on More Subjects}

As part of contribution 7 , the option of eliminating the need for repetitive training was investigated by pre-training a series of classifiers on a wide range of data samples from a single person, and testing them on a diverse population of volunteers to cover more variations. This section covers contribution 7 , and presents the results obtained by testing the system on more subjects.

\subsubsection{Experiments by Other Subjects}

For the purpose of results evaluation and validation, more volunteers were asked to capture a few CSI data by performing the same sequence of sitting and standing activities, without recording video.

Three members of the DSP lab at Carleton University volunteered to perform some sitting and standing activities and successfully recorded two or three CSI captures that followed the proposed data collection model. Their captures were used to test if the proposed solution works on other subjects. Table 23 below summarizes information and body characteristics about the subjects. Subjects M and J collected two CSI captures, whereas $\mathrm{H}$ collected three.

Table 23 Subject Information and Characteristics

\begin{tabular}{|c|c|c|c|c|c|}
\hline Number & Subject & Gender & Age (years) & Height (cm) & Weight (kg) \\
\hline $\mathbf{1}$ & I & Female & 25 & 160 & 57 \\
\hline $\mathbf{2}$ & M & Female & 26 & 170 & 57 \\
\hline $\mathbf{3}$ & J & Male & 29 & 180 & 59 \\
\hline $\mathbf{4}$ & H & Male & 24 & 172 & 64 \\
\hline
\end{tabular}

As such, four new data sets were obtained: 
1) a data set containing the data collected by subject $M$;

2) a data set containing the data collected by subject J;

3) a data set containing the data collected by subject $\mathrm{H}$; and

4) a data set combining the data collected by the three other subjects (the "other" data set), at random room locations, with the transmitter mounted at the ceiling.

Subject M's data set contained 137 samples before applying class balancing, and 371 after. Subject J's data set contained 136 samples before applying class balancing, and 359 after. Subject H's data set contained 177 samples before applying class balancing, and 500 after. The "other" data set contained 450 samples before applying class balancing, and 1,216 after.

Appendix E presents samples of the signals produced by having different subjects perform the same sequence of sitting and standing activities. It shows how the pattern in CSI captures is influenced by the body characteristics of the subjects.

\subsubsection{Dependence of Classification Performance on Subjects}

To study the influence of subject body characteristics on the classification performance, the classifiers were trained on the data sets acquired by individual subjects. As shown in Table 24, some classifiers were influenced as they performed differently on the different data sets. For example, the quadratic discriminant analysis method performed relatively different for each data set. It achieved an accuracy of $67.7 \%$ for subject $\mathrm{M}, 33.4 \%$ for subject J, $99.4 \%$ for subject $\mathrm{H}$, and $89.7 \%$ for the combined data set. Most classifiers performance degraded for the combined data set, which is expected as it is larger in size. 
Table 24 Classification Results for Individual Subjects, Dependent on the Subject

\begin{tabular}{|c|c|c|c|c|c|}
\hline \multirow[b]{2}{*}{$\begin{array}{l}\text { Classification } \\
\text { Method }\end{array}$} & \multirow[b]{2}{*}{ Type } & \multicolumn{4}{|c|}{ Accuracy (\%) } \\
\hline & & $\begin{array}{c}\text { Subject } \\
\text { M }\end{array}$ & $\begin{array}{c}\text { Subject } \\
\mathbf{J}\end{array}$ & $\begin{array}{c}\text { Subject } \\
\text { H }\end{array}$ & $\begin{array}{l}\text { Subject M, J, H } \\
\text { Combined }\end{array}$ \\
\hline \multirow{3}{*}{$\begin{array}{l}\text { Decision Trees } \\
\text { (DTs) }\end{array}$} & Fine & 93.5 & 95.3 & 97.4 & 90.5 \\
\hline & Medium & 93.5 & 95.3 & 97.4 & 87.0 \\
\hline & Coarse & 89.5 & 87.7 & 88.6 & 74.1 \\
\hline \multirow{2}{*}{$\begin{array}{l}\text { Discriminant } \\
\text { Analysis (DA) }\end{array}$} & Linear & 83.8 & 87.5 & 87.4 & 72.9 \\
\hline & Quadratic & 67.7 & 33.4 & 99.4 & 89.7 \\
\hline \multirow{2}{*}{$\begin{array}{l}\text { Naïve Bayes } \\
\text { (NB) }\end{array}$} & Gaussian & Failed & Failed & 99.4 & 89.7 \\
\hline & Kernel & 99.5 & 99.4 & 100 & 95.3 \\
\hline \multirow{6}{*}{$\begin{array}{c}\text { Support Vector } \\
\text { Machines } \\
\text { (SVMs) }\end{array}$} & Linear & 95.4 & 96.1 & 98.2 & 92.2 \\
\hline & Quadratic & 98.4 & 97.2 & 99.2 & 97.1 \\
\hline & Cubic & 97.6 & 97.5 & 99.2 & 97.4 \\
\hline & Fine Gaussian & 94.3 & 91.1 & 98.8 & 90.4 \\
\hline & Medium Gaussian & 100 & 100 & 100 & 99.8 \\
\hline & Coarse Gaussian & 90.0 & 95.5 & 95.2 & 88.2 \\
\hline \multirow{6}{*}{$\begin{array}{c}\text { K-Nearest } \\
\text { Neighbours } \\
\text { (KNN) }\end{array}$} & Fine & 80.9 & 81.9 & 86.8 & 82.8 \\
\hline & Medium & 72.0 & 69.4 & 74.0 & 71.7 \\
\hline & Coarse & 66.8 & 63.0 & 66.2 & 60.9 \\
\hline & Cosine & 84.9 & 93.6 & 95.2 & 88.6 \\
\hline & Cubic & 71.4 & 70.5 & 72.4 & 71.1 \\
\hline & Weighted & 71.7 & 68.5 & 74.0 & 71.5 \\
\hline \multirow{5}{*}{$\begin{array}{l}\text { Ensemble } \\
\text { Classifiers }\end{array}$} & Boosted Trees & 33.7 & 33.4 & 33.6 & 94.0 \\
\hline & Bagged Trees & 95.1 & 98.6 & 98.2 & 97.0 \\
\hline & $\begin{array}{c}\text { Subspace } \\
\text { Discriminant }\end{array}$ & 92.5 & 92.5 & 98.2 & 93.0 \\
\hline & Subspace KNN & 84.9 & 86.6 & 93.4 & 88.1 \\
\hline & RUSBoosted Trees & 33.7 & 45.1 & 33.6 & 87.3 \\
\hline
\end{tabular}

The medium Gaussian SVM outperformed the other classifiers by achieving perfect performances for all subjects, and $99.8 \%$ on the combined data set. However, these variations can be caused by other factors such as activity location, environmental changes, and noise level as well. Another factor is the integrity of the data collected by the other subjects as one cannot guarantee that they performed the required exercise as expected. If 
one can verify that these differences were really caused by individual body characteristics, that would mean that the system needs to either be repeatedly trained and configured for users upon deployment (subject dependence), or be trained on a huge data set collected by a diverse population of subjects (subject independence). The first option would lead to a system in which the performance depends on the subject; while the second option would lead to a system in which the performance is independent of the subject.

Tables 25, 26, 27 and 28 show the confusion matrices of the predictions for the data sets collected by subject $M$, subject $J$, subject $H$, and the combined data set, respectively.

Table 25 Confusion Matrix Summarizing the Medium Gaussian SVM Predictions for the Data Set Collected by Subject M

\begin{tabular}{|c|c|c|c|c|}
\hline \multirow{3}{*}{ 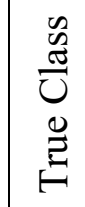 } & Still & 123 & 0 & 0 \\
\hline & Sitting-down & 0 & 125 & 0 \\
\hline & Standing-up & 0 & 0 & 123 \\
\hline & & Still & Sitting-down & Standing-up \\
\hline & & \multicolumn{3}{|c|}{ Predicted Class } \\
\hline
\end{tabular}

Table 26 Confusion Matrix Summarizing the Medium Gaussian SVM Predictions for the Data Set Collected by Subject $\mathbf{J}$

\begin{tabular}{|c|c|c|c|c|}
\hline \multirow{3}{*}{ 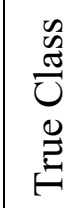 } & Still & 120 & 0 & 0 \\
\hline & Sitting-down & 0 & 119 & 0 \\
\hline & Standing-up & 0 & 0 & 120 \\
\hline & & Still & Sitting-down & Standing-up \\
\hline & & \multicolumn{3}{|c|}{ Predicted Class } \\
\hline
\end{tabular}


Table 27 Confusion Matrix Summarizing the Medium Gaussian SVM Predictions for the Data Set Collected by Subject $\mathrm{H}$

\begin{tabular}{|c|c|c|c|c|}
\hline \multirow{3}{*}{ 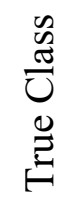 } & Still & 166 & 0 & 0 \\
\hline & Sitting-down & 0 & 166 & 0 \\
\hline & Standing-up & 0 & 0 & 168 \\
\hline & & Still & Sitting-down & Standing-up \\
\hline & & \multicolumn{3}{|c|}{ Predicted Class } \\
\hline
\end{tabular}

Table 28 Confusion Matrix Summarizing the Medium Gaussian SVM Predictions for the "other"

Data Set, Combining Data Collected by Subjects M, J, and H

\begin{tabular}{|c|c|c|c|c|}
\hline \multirow{3}{*}{ 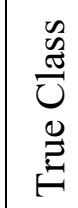 } & Still & 407 & 2 & 0 \\
\hline & Sitting-down & 0 & 401 & 0 \\
\hline & Standing-up & 0 & 0 & 406 \\
\hline & & Still & Sitting-down & Standing-up \\
\hline & & \multicolumn{3}{|c|}{ Predicted Class } \\
\hline
\end{tabular}

As the accuracies suggest, the confusion matrices also reflect perfect classification performances for the data sets of the three subjects, and an almost perfect performance for the combined data set. All samples were correctly classified for subject M, J, and H. Only two samples of the combined data set were wrongly classified.

\subsubsection{Independence of Classification Performance from Subjects}

The classifiers were trained on a data set combining all of the collected data: the "floor", "ceiling", "floor + ceiling", and "other" data sets. To see how the classifiers would perform on the data set collected by the volunteers, training the classifiers on it was considered as well. This was done to aim towards a trained-once activity recognition and 
monitoring system, which would be able to classify the CSI data corresponding to ADLs, under any circumstances.

Table 29 Classification Results of Training of Different Data Sets, Independent of the Subject

\begin{tabular}{|c|c|c|c|c|c|c|}
\hline \multirow{2}{*}{$\begin{array}{l}\text { Classification } \\
\text { Method }\end{array}$} & \multirow[b]{2}{*}{ Type } & \multicolumn{5}{|c|}{ Accuracy (\%) } \\
\hline & & Floor & Ceiling & $\begin{array}{l}\text { Floor + } \\
\text { Ceiling }\end{array}$ & Other & $\begin{array}{c}\text { All } \\
\text { Combined }\end{array}$ \\
\hline \multirow{3}{*}{$\begin{array}{c}\text { Decision } \\
\text { Trees (DTs) }\end{array}$} & Fine & 68.1 & 65.7 & 64.4 & 90.5 & 62.7 \\
\hline & Medium & 62.5 & 59.2 & 59.1 & 87.0 & 57.3 \\
\hline & Coarse & 56.3 & 55.0 & 55.2 & 74.1 & 54.8 \\
\hline \multirow{2}{*}{$\begin{array}{l}\text { Discriminant } \\
\text { Analysis (DA) }\end{array}$} & Linear & 54.8 & 54.2 & 52.5 & 72.9 & 52.4 \\
\hline & Quadratic & 61.1 & 60.1 & 58.2 & 89.7 & 57.5 \\
\hline \multirow{2}{*}{$\begin{array}{l}\text { Naïve Bayes } \\
\text { (NB) }\end{array}$} & Gaussian & 61.1 & 60.1 & 58.2 & 89.7 & 57.5 \\
\hline & Kernel & 72.2 & 72.6 & 68.7 & 95.3 & 68.5 \\
\hline \multirow{6}{*}{$\begin{array}{l}\text { Support } \\
\text { Vector } \\
\text { Machines } \\
\text { (SVMs) }\end{array}$} & Linear & 67.0 & 65.9 & 62.4 & 92.2 & 62.2 \\
\hline & Quadratic & 94.5 & 93.3 & 92.2 & 97.1 & 92.1 \\
\hline & Cubic & 97.6 & 96.6 & 97.0 & 97.4 & 97.0 \\
\hline & $\begin{array}{c}\text { Fine } \\
\text { Gaussian }\end{array}$ & 90.8 & 88.6 & 90.2 & 90.4 & 89.8 \\
\hline & $\begin{array}{l}\text { Medium } \\
\text { Gaussian }\end{array}$ & 98.4 & 97.6 & 97.7 & 99.8 & 97.6 \\
\hline & $\begin{array}{c}\text { Coarse } \\
\text { Gaussian }\end{array}$ & 70.7 & 68.7 & 66.7 & 88.2 & 67.1 \\
\hline \multirow{6}{*}{$\begin{array}{c}\text { K-Nearest } \\
\text { Neighbours } \\
\text { (KNN) }\end{array}$} & Fine & 80.9 & 80.0 & 79.7 & 82.8 & 79.6 \\
\hline & Medium & 72.3 & 71.2 & 71.5 & 71.7 & 71.2 \\
\hline & Coarse & 57.2 & 55.3 & 56.8 & 60.9 & 56.7 \\
\hline & Cosine & 85.8 & 84.2 & 85.5 & 88.6 & 85.1 \\
\hline & Cubic & 71.3 & 70.1 & 70.8 & 71.1 & 70.5 \\
\hline & Weighted & 72.1 & 71.6 & 71.2 & 71.5 & 71.1 \\
\hline \multirow{5}{*}{$\begin{array}{l}\text { Ensemble } \\
\text { Classifiers }\end{array}$} & $\begin{array}{c}\text { Boosted } \\
\text { Trees }\end{array}$ & 66.2 & 63.0 & 61.0 & 94.0 & 60.3 \\
\hline & Bagged Trees & 96.0 & 94.8 & 94.6 & 97.0 & 94.3 \\
\hline & $\begin{array}{c}\text { Subspace } \\
\text { Discriminant }\end{array}$ & 64.5 & 63.7 & 59.1 & 93.0 & 59.4 \\
\hline & $\begin{array}{c}\text { Subspace } \\
\text { KNN }\end{array}$ & 86.8 & 84.8 & 85.2 & 88.1 & 85.0 \\
\hline & $\begin{array}{c}\text { RUSBoosted } \\
\text { Trees }\end{array}$ & 63.5 & 60.6 & 59.8 & 87.3 & 58.2 \\
\hline
\end{tabular}


Table 29 summarizes the classification accuracies achieved by training the classifiers on the different data sets (see section 5.3.1). Note that the reason for the values in the "Floor", "Ceiling", and "Floor + Ceiling" columns to differ slightly from the values in the previous tables is that this experiment was carried out at a later point in time.

Table 30 Classification Results of Training on "floor" Data Set and Testing on "other" Data Set

\begin{tabular}{|c|c|c|c|}
\hline \multirow{2}{*}{$\begin{array}{l}\text { Classification } \\
\text { Method }\end{array}$} & \multirow[b]{2}{*}{ Type } & \multicolumn{2}{|c|}{ Accuracy $(\%)$} \\
\hline & & $\begin{array}{c}\text { Training on } \\
\text { "floor" Data Set }\end{array}$ & $\begin{array}{c}\text { Testing on } \\
\text { “other" Data Set }\end{array}$ \\
\hline \multirow{3}{*}{$\begin{array}{l}\text { Decision Trees } \\
\text { (DTs) }\end{array}$} & Fine & 68.1 & 42.1 \\
\hline & Medium & 62.5 & 37.1 \\
\hline & Coarse & 56.3 & 42.9 \\
\hline \multirow{2}{*}{$\begin{array}{l}\text { Discriminant } \\
\text { Analysis (DA) }\end{array}$} & Linear & 54.8 & 42.8 \\
\hline & Quadratic & 61.1 & 42.3 \\
\hline \multirow{2}{*}{ Naïve Bayes (NB) } & Gaussian & 61.1 & 42.3 \\
\hline & Kernel & 72.2 & 47.0 \\
\hline \multirow{6}{*}{$\begin{array}{c}\text { Support Vector } \\
\text { Machines (SVMs) }\end{array}$} & Linear & 67.0 & 33.2 \\
\hline & Quadratic & 94.5 & 43.8 \\
\hline & Cubic & 97.6 & 39.9 \\
\hline & Fine Gaussian & 90.8 & 33.6 \\
\hline & Medium Gaussian & 98.4 & 35.2 \\
\hline & Coarse Gaussian & 70.7 & 45.0 \\
\hline \multirow{6}{*}{$\begin{array}{c}\text { K-Nearest } \\
\text { Neighbours } \\
\text { (KNN) }\end{array}$} & Fine & 80.9 & 39.6 \\
\hline & Medium & 72.3 & 39.5 \\
\hline & Coarse & 57.2 & 34.9 \\
\hline & Cosine & 85.8 & 35.6 \\
\hline & Cubic & 71.3 & 36.8 \\
\hline & Weighted & 72.1 & 39.1 \\
\hline \multirow{5}{*}{$\begin{array}{l}\text { Ensemble } \\
\text { Classifiers }\end{array}$} & Boosted Trees & 66.2 & 38.0 \\
\hline & Bagged Trees & 96.0 & 43.4 \\
\hline & Subspace Discriminant & 64.5 & 32.9 \\
\hline & Subspace KNN & 86.8 & 40.7 \\
\hline & RUSBoosted Trees & 63.5 & 36.9 \\
\hline \multicolumn{2}{|c|}{ AVERAGE DIFFERENCE } & \multicolumn{2}{|c|}{34.5} \\
\hline
\end{tabular}


Table 31 Classification Results of Training on "ceiling" Data Set and Testing on "other" Data Set

\begin{tabular}{|c|c|c|c|}
\hline \multirow[b]{2}{*}{ Classification Method } & \multirow[b]{2}{*}{ Type } & \multicolumn{2}{|c|}{ Accuracy $(\%)$} \\
\hline & & $\begin{array}{c}\text { Training on } \\
\text { “ceiling” Data Set }\end{array}$ & $\begin{array}{c}\text { Testing on } \\
\text { “other" Data Set }\end{array}$ \\
\hline \multirow{3}{*}{ Decision Trees (DTs) } & Fine & 65.7 & 29.7 \\
\hline & Medium & 59.2 & 34.4 \\
\hline & Coarse & 55.0 & 44.1 \\
\hline \multirow{2}{*}{$\begin{array}{l}\text { Discriminant } \\
\text { Analysis (DA) }\end{array}$} & Linear & 54.2 & 43.0 \\
\hline & Quadratic & 60.1 & 41.5 \\
\hline \multirow{2}{*}{ Naïve Bayes (NB) } & Gaussian & 60.1 & 41.5 \\
\hline & Kernel & 72.6 & 52.5 \\
\hline \multirow{6}{*}{$\begin{array}{c}\text { Support Vector } \\
\text { Machines (SVMs) }\end{array}$} & Linear & 65.9 & 36.7 \\
\hline & Quadratic & 93.3 & 50.4 \\
\hline & Cubic & 96.6 & 50.7 \\
\hline & Fine Gaussian & 88.6 & 33.6 \\
\hline & Medium Gaussian & 97.6 & 34.4 \\
\hline & Coarse Gaussian & 68.7 & 49.1 \\
\hline \multirow{6}{*}{$\begin{array}{c}\text { K-Nearest } \\
\text { Neighbours }(\text { KNN) }\end{array}$} & Fine & 80.0 & 39.7 \\
\hline & Medium & 71.2 & 37.1 \\
\hline & Coarse & 55.3 & 32.5 \\
\hline & Cosine & 84.2 & 37.4 \\
\hline & Cubic & 70.1 & 36.0 \\
\hline & Weighted & 71.6 & 36.8 \\
\hline \multirow{5}{*}{ Ensemble Classifiers } & Boosted Trees & 63.0 & 37.9 \\
\hline & Bagged Trees & 94.8 & 45.4 \\
\hline & $\begin{array}{c}\text { Subspace } \\
\text { Discriminant }\end{array}$ & 63.7 & 33.5 \\
\hline & Subspace KNN & 84.8 & 45.9 \\
\hline & RUSBoosted Trees & 60.6 & 31.9 \\
\hline \multicolumn{2}{|c|}{ AVERAGE DIFFERENCE } & \multicolumn{2}{|c|}{32.6} \\
\hline
\end{tabular}

Three sets of models were generated by training the classifiers on the "floor", "ceiling", and "floor + ceiling" data sets, respectively. All models were trained with 5-fold cross validation to protect the learners against overfitting by partitioning the data sets into five folds and estimating the classification accuracy on each fold. Afterwards, each of the 
trained models was tested using the "other" data set, which consisted of the data collected by the three volunteers, with the receiver on the ground and the transmitter at the ceiling.

Since the activities performed by the other subjects were conducted with the transmitter mounted at the ceiling, one would expect the testing performance to improve when training the classifiers on the "ceiling" data set. As can be seen in Tables 30 and 31 above, this was indeed the case. The average difference between the training and testing accuracies was $34.5 \%$ when training on the "floor" data set, while it was reduced to $32.6 \%$ when training on the "ceiling" data set.

In addition, training the classifiers on a larger data set, combining both the "floor" and "ceiling" data sets reduced the average difference even more. When training on the "floor + ceiling" data set, the average difference between the training and testing accuracies was approximately $30.1 \%$ (see Table 32 below).

As observed in Tables 30, 31, and 32 above, the performance of the classifiers significantly dropped when tested on the data set collected by other subjects. These changes in performance can be due to a number of reasons, including signal/time drift, subject bias, and classifier overfitting. Factors such as time, environmental changes, noise, and device calibration contribute to signal drift. On the other hand, the system appeared unable to detect sitting-down and standing-up actions by different people, which it was not trained on. Looking closely at the models exported from MATLAB's Classification Learner, the prediction functions, used to predict the classes of test data samples, were noticed to generate ahead of validation, meaning that the system would not be protected against overfitting. 
Table 32 Classification Results of Training on "floor + ceiling" and Testing on "other" Data Set

\begin{tabular}{|c|c|c|c|}
\hline \multirow[b]{2}{*}{$\begin{array}{l}\text { Classification } \\
\text { Method }\end{array}$} & \multirow[b]{2}{*}{ Type } & \multicolumn{2}{|c|}{ Accuracy $(\%)$} \\
\hline & & $\begin{array}{l}\text { Training on "floor } \\
+ \text { ceiling" Data Set }\end{array}$ & $\begin{array}{c}\text { Testing on } \\
\text { “other" Data Set }\end{array}$ \\
\hline \multirow{3}{*}{$\begin{array}{c}\text { Decision } \\
\text { Trees (DTs) }\end{array}$} & Fine & 64.4 & 33.3 \\
\hline & Medium & 59.1 & 37.8 \\
\hline & Coarse & 55.2 & 45.0 \\
\hline \multirow{2}{*}{$\begin{array}{l}\text { Discriminant } \\
\text { Analysis (DA) }\end{array}$} & Linear & 52.5 & 43.8 \\
\hline & Quadratic & 58.2 & 43.1 \\
\hline \multirow{2}{*}{$\begin{array}{l}\text { Naïve Bayes } \\
\text { (NB) }\end{array}$} & Gaussian & 58.2 & 43.1 \\
\hline & Kernel & 68.7 & 53.7 \\
\hline \multirow{6}{*}{$\begin{array}{l}\text { Support } \\
\text { Vector } \\
\text { Machines } \\
\text { (SVMs) }\end{array}$} & Linear & 62.4 & 33.5 \\
\hline & Quadratic & 92.2 & 54.4 \\
\hline & Cubic & 97.0 & 50.3 \\
\hline & Fine Gaussian & 90.2 & 33.6 \\
\hline & Medium Gaussian & 97.7 & 39.5 \\
\hline & Coarse Gaussian & 66.7 & 50.1 \\
\hline \multirow{6}{*}{$\begin{array}{c}\text { K-Nearest } \\
\text { Neighbours } \\
\text { (KNN) }\end{array}$} & Fine & 79.7 & 41.0 \\
\hline & Medium & 71.5 & 38.7 \\
\hline & Coarse & 56.8 & 31.7 \\
\hline & Cosine & 85.5 & 41.0 \\
\hline & Cubic & 70.8 & 37.3 \\
\hline & Weighted & 71.2 & 38.7 \\
\hline \multirow{5}{*}{$\begin{array}{l}\text { Ensemble } \\
\text { Classifiers }\end{array}$} & Boosted Trees & 61.0 & 39.0 \\
\hline & Bagged Trees & 94.6 & 51.2 \\
\hline & Subspace Discriminant & 59.1 & 33.1 \\
\hline & Subspace KNN & 85.2 & 46.1 \\
\hline & RUSBoosted Trees & 59.8 & 37.0 \\
\hline \multicolumn{2}{|c|}{ AVERAGE DIFFERENCE } & \multicolumn{2}{|c|}{30.1} \\
\hline
\end{tabular}




\subsubsection{Deep Learning Results}

This section will cover contribution 8 from the list of contributions in section 1.4. Contribution 8 is related to the implementation of a deep learning neural network to recognize and classify sitting and standing activities. Two versions of the data in the "floor + ceiling" data set were generated. One before segmentation (180 samples/sequences), and another after segmenting the data into 20-packet segments (12,057 samples/sequences). The first version is useful for testing a sequence-to-sequence model of the network, where the network takes a time series as the input and outputs a sequence of labels (i.e. predictions). The inputted time series consisted of the acquired and preprocessed 90 -second CSI signals. The second version of the data is useful for testing a sequence-to-label model of the designed network, where the network takes a time series as the input and outputs a label at each iteration. In this case, the inputted time series consisted of the preprocessed and segmented 1-second CSI signals.

In some real cases, different people may perform the same activity differently. For all supervised learning, if activities performed by a subject " $\mathrm{A}$ " do not appear in training, the data collected by "A" will be harder to predict during testing. To verify this, the proposed network was trained with the data collected by one person and validate it with the data collected by three other subjects. The training data set was collected by one subject, who performed many variations of sitting and standing speeds and styles.

The network was tested by training and validating it using three activity classes, and four activity classes. Both seated and standstill activities were first joined into the "still" class, and both sitting-down and standing-up activities were kept in their respective classes. Then, the still activities were split into two classes: "seated" and "standstill". This 
resulted with four activity classes: "seated", "standstill”, "sitting-down”, and standing-up". Both seated and standstill activities are very similar in the sense that they are stationary. On the other hand, both sitting-down and standing-up movements contain the same actions in reverse order. Therefore, it is essential to distinguish among them.

\subsubsection{Sequence-to-Sequence BiLSTM Network}

A sequence-to-sequence model of the BiLSTM network, where the network takes a time series as the input and outputs a sequence of labels (i.e. predictions) was explored. The inputted time series consisted of the acquired and preprocessed 90 -second CSI signals. Dealing with the data without segmentation provides a data set of 90 -second sequences. By setting the maximum number of epochs to 100 , with a minimum batch size of 20, having a data set of 180 sequences, each epoch will consist of nine iterations, for a total of 900 training iterations. The validation data set, on the other hand, contains only seven sequences.

For the scenario with three activity classes, this model of the BiLSTM network achieved a classification accuracy of $85.1 \%$. It achieved a classification accuracy of $75.7 \%$ with four activity classes.

\subsubsection{Sequence-to-Label BiLSTM Network}

A sequence-to-label model of the BiLSTM network, where the network takes a time series as the input and outputs a label at each iteration was explored. In this case, the inputted time series consisted of the preprocessed and segmented 1-second CSI signals. The CSI signals were segmented into 20-packet windows and accordingly labelled. A window length of 20 was chosen because some sitting-down and standing-up activities take 
about 1 second (i.e. about 20 packets) to complete. To segment the signals, the starting point for each activity in the sequence was first identified. Once the appropriate starting timestamp/packet for an activity was located, the signal was segmented into 20-packet segments until the next event's timestamp was reached.

Data segmentation split the CSI sequences into 20-packet (1-second) sequences; thus, providing a much larger data set. By setting the maximum number of epochs to 100 , with a minimum batch size of 20, having a data set of 12,057, each epoch will consist of 602 iterations, for a total of 60,200 training iterations. The validation data set, on the other hand, contains 450 sequences.

For the scenario with three activity classes, the BiLSTM network achieved a classification accuracy of $90.7 \%$. For the scenario with four activity classes, the BiLSTM network achieved a classification accuracy of 55.6\%. Since accuracy is not a good measure of classification performance in the case of imbalanced classes, Table 33 and Table 34 show the confusion matrices as they are more representative of the performance. Table 33 displays the confusion matrix for predicting three classes of activities, while Table 34 displays the confusion matrix for predicting four classes of activities.

Table 33 Confusion Matrix Displaying the Validation Predictions of a Sequence-to-Label BiLSTM Network, with Three Activity Classes

\begin{tabular}{|c|c|c|c|c|}
\hline \multirow{2}{*}{$\underset{\Xi}{\tilde{J}}$} & Still & 407 & 0 & 2 \\
\cline { 2 - 5 }$\underset{\Xi}{\Xi}$ & Standing-up & 20 & 0 & 0 \\
\cline { 2 - 5 } & Still & 19 & 0 & 1 \\
\hline \multirow{2}{*}{} & Still & Sitting-down & Standing-up \\
\cline { 2 - 5 } & \multicolumn{3}{|c|}{ Predicted Class } \\
\hline
\end{tabular}


Table 34 Confusion Matrix Displaying the Validation Predictions of a Sequence-to-Label BiLSTM

Network, with Four Activity Classes

\begin{tabular}{|c|c|c|c|c|c|}
\hline \multirow{4}{*}{ 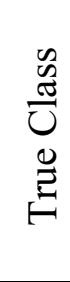 } & Seated & 182 & 0 & 2 & 57 \\
\hline & Sitting-down & 11 & 1 & 1 & 8 \\
\hline & Standing-up & 16 & 0 & 2 & 2 \\
\hline & Standstill & 101 & 0 & 2 & 65 \\
\hline & & Seated & Sitting-down & Standing-up & Standstill \\
\hline & & & Predict & d Class & \\
\hline
\end{tabular}

Due to the nature of the employed data collection, the collected data contained more samples belonging to the "still", "seated", and "standstill" classes, and, as such, the data set was imbalanced. As balancing the classes in time series data can become complex, and due to time constraints, this item was left for future work. The above confusion matrices show that the network is affected by the highly imbalanced structure of the training set. This could also be due to the size of the training set as it deep learning networks usually need significantly more data to train, or the proposed technique is not suitable for the problem. Table 35 summarizes all of the obtained results. The sequence-to-label model achieves a maximum accuracy of $90.7 \%$ at recognizing three activity classes: still, sittingdown, and standing-up.

Table 35 Summary of Results from Both Sequence-to-Sequence and Sequence-to-Label Models of the BiLSTM Network

\begin{tabular}{|c|c|c|c|c|c|}
\hline \multirow{2}{*}{ Test } & Model & $\begin{array}{c}\text { Training } \\
\text { Samples }\end{array}$ & $\begin{array}{c}\text { Testing } \\
\text { Samples }\end{array}$ & $\begin{array}{c}\text { Activity } \\
\text { Classes }\end{array}$ & $\begin{array}{c}\text { Accuracy } \\
\text { (\%) }\end{array}$ \\
\hline $\mathbf{1}$ & Sequence-to-Sequence & 180 & 7 & 3 & 85.1 \\
\hline $\mathbf{2}$ & Sequence-to-Sequence & 180 & 7 & 4 & 75.7 \\
\hline $\mathbf{3}$ & Sequence-to-Label & 12,057 & 450 & 3 & 90.7 \\
\hline $\mathbf{4}$ & Sequence-to-Label & 12,057 & 450 & 4 & 55.6 \\
\hline
\end{tabular}




\section{Chapter 6: Conclusion}

This chapter concludes the thesis with a discussion of the main research findings and includes some closing remarks and possible extensions of the work.

\subsection{Summary of Related Work}

This thesis utilizes the Wi-Fi devices provided by Aerial Technologies, as in $[1,33]$, and proposes a HAR system that could potentially be used to recognize ADLs. It extends the previous application of Wi-Fi CSI analysis for motion and location detection to not only distinguish between a no motion activity and motion activities, as it is the case in [33], but to also allow for two very similar motions of standing-up and sitting-down to be distinguished from one another. The sensor used in [33] was simply a motion detector, while this thesis introduces a HAR sensor that can capture movement/motion patterns. Although [42 - 47] distinguish among ADL, [42, 43] do not distinguish between similar movements, and only [44, 47] apply deep learning.

This work focuses on the recognition of the two similar dynamic motions of sittingdown and standing-up. Furthermore, [42 - 47] apply more complex preprocessing algorithms. This thesis aims at simplifying the preprocessing methodology, while still achieving an acceptable recognition/classification performance. The applied feature extraction and traditional machine learning classification, in this work, have demonstrated the ability to extend the previous application of Wi-Fi CSI analysis for motion and location detection to not only distinguish between a no motion activity and two motion activities, but to also allow for two very similar motions of standing-up and sitting-down to be distinguished from one another. 
Traditional machine learners as well as a deep learning network were tested to classify sitting and standing activities. Table 36 compares the performance achieved by this work, at recognizing sitting and standing activities, with some state-of-the-art studies. The top three rows of the table represent the results presented in this thesis, and the support papers in comparison to other works.

Table 36 Comparison of Classification Performances with Related Work

\begin{tabular}{|c|c|c|c|}
\hline Reference & Movement & Classification Method & Average Accuracy (\%) \\
\hline New [1] & Dynamic & Traditional Machine Learning & $\mathbf{9 8 . 4}$ \\
\hline New & Dynamic & Traditional Machine Learning & $\mathbf{9 8 . 2}$ \\
\hline New & Dynamic & Deep Learning & $\mathbf{8 7 . 9}$ \\
\hline$[42]$ & Static & Traditional Machine Learning & 92 \\
\hline$[43]$ & Static & Traditional Machine Learning & 96 \\
\hline$[44]$ & Dynamic & Deep Learning & over 80 \\
\hline$[45]$ & Dynamic & Traditional Machine Learning & 92 \\
\hline$[46]$ & Dynamic & Traditional Machine Learning & 95.3 \\
\hline$[47]$ & Dynamic & Deep Learning & 97 \\
\hline
\end{tabular}

\subsection{Discussion of Results}

CSI data were acquired by performing a sequence of still, sitting-down and standingup action activities, preprocessed the acquired signals, extracted features, and labelled the data samples for traditional, supervised machine learning. Four different data sets were generated using the acquired Wi-Fi CSI signals: "preliminary", "floor", "ceiling", and “floor + ceiling" (see section 5.3.1). The "preliminary" data set contained data collected by a single subject, at a single room location, with both Wi-Fi devices placed on the ground. The so-called "preliminary" data set was utilized to perform basic test cases for the proposed contributions. The "floor" data set contained data collected by a single subject, at various room locations, with both Wi-Fi devices placed on the floor. The "ceiling" data 
set included data collected by a single subject, at various room locations, with one Wi-Fi device mounted at the ceiling and the other placed on the floor. The "floor + ceiling" data set simply combined both of the "floor" and "ceiling" data sets together.

MATLAB's Classification Learner was used to train various classifiers on the CSI data (see section 4.5.1). The classifiers were trained and tested using 5-fold cross validation. Basic tests, with minimal data preprocessing (only filtering), were performed on the so-called "preliminary" data set. As such, the medium Gaussian SVM achieved a highest accuracy of $98.4 \%$. The manner at which SVMs operate explains the obtained results. SVMs determine an optimal hyperplane that defines a clear gap between the data points in terms of space [48].

As part of data preprocessing, the effects of standardizing/normalizing the CSI measurements and scaling the extracted features, on classification accuracies, were studied (see section 5.2). The medium Gaussian SVM achieved a highest accuracy of $98.8 \%$ for the so-called "preliminary" data set, by applying z-score standardization over subcarrier indices for each timestamp/packet, and scaling the features at all levels of the feature extraction paradigm.

By testing and reporting results on the "floor", "ceiling", and "floor + ceiling" data sets, different activity locations and device placements were examined (see section 5.3). The best classification performance was achieved by training the classifiers on the "floor" data set. The medium Gaussian SVM achieved an accuracy of 98.5\% for the "floor" data set, $97.7 \%$ for the "ceiling" data set, and $97.8 \%$ for the "floor + ceiling" data set. The proposed solution was able to successfully distinguish between the three cases: stationary 
activities such as remaining seated or standstill, and two dynamic activities such as sittingdown or standing-up that were performed by one person. This goes beyond detecting motion as the system was able to distinguish between two different movements.

Time repeatability and drift were also tested (see section 5.4) by running the classifiers on data sets collected on different days ("day 1" and "day 2"), and again on data sets collected on the same day, within a short period of time ("capture 1" and "capture 2"). In terms of repeatability, the obtained results showed that the proposed solution performed as good, regardless of the time/date the tests and data collection. In terms of drift, the obtained results showed that the proposed solution was influenced by drift and/or other factors. The classification performance of the system degraded when training it on one data set, with 5-fold cross validation, and testing it on another data set. The performance was degraded despite the amount of time passed between the collection of the two data sets.

Most of the acquired CSI data were captured by a single person performing the proposed sequence of sitting-down and standing-up activities, using various experimental setups. More data were collected by other subjects to cover different variations of age, gender, height, and weight. Other means of validation, such as holdout validation and separate testing data sets, were also investigated to see to what extent can the system proceed without degrading the performance. The different classifiers were trained on data collected by the preliminary subject (the author), and tested using data collected by other subjects. This was done in an effort to explore the user independence of the proposed methods. Unfortunately, the classification performance was significantly degraded when training the classifiers on the data collected by one subject and testing on the data collected by other subjects. This can be a result of many factors, such as overfitting the training data, 
time/signal drifting, environmental changes, or the differing body characteristics of subjects. Another issue that comes into question is the integrity of the data collected by the other subjects, as one cannot guarantee that they precisely followed the experimental instructions. Thus, the resulting CSI signals might be largely affected and not similar, in pattern, to those that were used during the training process.

A BiLSTM network was designed to perform deep learning on the acquired Wi-Fi CSI data (see section 4.5.2 and 5.5.4). The network was tested for both sequence-tosequence and sequence-to-label models of classification. The inputted sequences were constituted of time series of 21 attributes. Furthermore, the two models were tested using three activity classes (still, sitting-down, and standing-up), as well as four activity classes (seated, standstill, sitting-down, and standing-up). The seated and standstill activities are static, while sitting-down and standing-up are dynamic activities.

The sequence-to-sequence model was trained on 180 labelled time series, and tested on seven time series. In this scenario, the network achieved a validation accuracy of $85.1 \%$ at classifying sitting and standing sequences, with three activity classes. It achieved a validation accuracy of $75.7 \%$ at classifying sitting and standing sequences, with four activity classes.

The sequence-to-label model was trained on 12,057 labelled time series, and tested on 450 time series. In this scenario, the network achieved a validation accuracy of $90.7 \%$ at classifying sitting and standing sequences, with three activity classes. It achieved a validation accuracy of $55.6 \%$ at classifying sitting and standing sequences, with four activity classes. 
The classification performance is expected to increase if the BiLSTM network was to be trained on a larger amount of data. Also, due to the data collection process, there is a class imbalance, where the still activities are majority, which could influence the classification performance. Since the nature of BiLSTM requires ordered data and most current approaches for data balancing do not provide solutions that maintain the order of data, the class imbalance issue will have to be studied in more detail and addressed as part of future work.

\subsection{Future Work}

In general, Wi-Fi seems an appropriate solution for capturing movements and nonstill activities. The proposed solution performs well on data that it was trained on, with 5fold cross validation, and data similar to the one that it was trained on, but not on data collected at other times or by other subjects. The performance of the system is then said to be user dependent, meaning that it would need to be continually trained in an adaptive machine learning process. In future work, the solution should improve in terms of subject dependence and independence from classification performance, by collecting more diverse data sets.

In the future, the addition of a stateful mechanism will be explored to improve the classification by distinguishing between standing still and seated, by tracking previous predictions to decide whether the subject is sitting still or standing still (C) 2019 [1]. If the mechanism detects that the person was standing-up before, he/she is likely standing still after. Also, one should aim towards recognizing other human activities such as walking. Finally, as part of future work, the potential for a hybrid classifier that combines the results from multiple classifiers will be explored to predict activities by favouring the most 
predicted class among all classifiers in order to potentially achieve an improved performance. 


\section{Appendices}

\section{Appendix A Summary of Data Samples}

This appendix contains a table summary of all of the conducted experiments, which provided the data for this work.

Table 37 Summary of Sitting and Standing Data Samples Collected under a 2D (Ground-Ground) Device Placement, by the Author

\begin{tabular}{|c|c|c|c|c|c|c|c|}
\hline \multicolumn{2}{|c|}{ Device Location } & \multirow{2}{*}{$\begin{array}{c}\text { Activity } \\
\text { Location } \\
\end{array}$} & \multirow{2}{*}{$\begin{array}{c}\text { Body } \\
\text { Orientation }\end{array}$} & \multicolumn{3}{|c|}{ Sit-Stand Style/Speed } & \multirow{2}{*}{ Total } \\
\hline $\mathbf{A P}$ & STA & & & 1 & 2 & 3 & \\
\hline \multirow{6}{*}{ Ground } & \multirow{6}{*}{ Ground } & \multirow{6}{*}{ Middle } & Front & 2 & 2 & 3 & 7 \\
\hline & & & Back & 2 & 2 & 3 & 7 \\
\hline & & & Towards AP & 3 & 2 & 3 & 8 \\
\hline & & & Towards STA & 3 & 2 & 2 & 7 \\
\hline & & & Left & 2 & 2 & 2 & 6 \\
\hline & & & Right & 2 & 2 & 3 & 7 \\
\hline \multirow{6}{*}{ Ground } & \multirow{6}{*}{ Ground } & \multirow{6}{*}{ Near AP } & Front & 1 & 1 & 1 & 3 \\
\hline & & & Back & 1 & 1 & 1 & 3 \\
\hline & & & Towards AP & 1 & 1 & 1 & 3 \\
\hline & & & Towards STA & 1 & 1 & 1 & 3 \\
\hline & & & Left & 1 & 1 & 1 & 3 \\
\hline & & & Right & 1 & 1 & 1 & 3 \\
\hline \multirow{6}{*}{ Ground } & \multirow{6}{*}{ Ground } & \multirow{6}{*}{ Near STA } & Front & 1 & 1 & 1 & 3 \\
\hline & & & Back & 1 & 1 & 1 & 3 \\
\hline & & & Towards AP & 1 & 1 & 1 & 3 \\
\hline & & & Towards STA & 1 & 1 & 1 & 3 \\
\hline & & & Left & 1 & 1 & 1 & 3 \\
\hline & & & Right & 1 & 1 & 1 & 3 \\
\hline \multirow{4}{*}{ Ground } & \multirow{4}{*}{ Ground } & \multirow{4}{*}{$\begin{array}{l}\text { Front-Left } \\
\text { Corner }\end{array}$} & Front & 1 & 1 & 1 & 3 \\
\hline & & & Back & 1 & 1 & 1 & 3 \\
\hline & & & Left & 1 & 1 & 1 & 3 \\
\hline & & & Right & 1 & 1 & 1 & 3 \\
\hline \multirow{4}{*}{ Ground } & \multirow{4}{*}{ Ground } & \multirow{4}{*}{$\begin{array}{c}\text { Back-Right } \\
\text { Corner }\end{array}$} & Front & 1 & 1 & 1 & 3 \\
\hline & & & Back & 1 & 1 & 1 & 3 \\
\hline & & & Left & 1 & 1 & 1 & 3 \\
\hline & & & Right & 1 & 1 & 1 & 3 \\
\hline \multicolumn{4}{|c|}{ TOTAL NUMBER OF DATA SAMPLES } & 34 & 32 & 36 & 102 \\
\hline
\end{tabular}


Table 38 Summary of Sitting and Standing Data Samples Collected under a 3D (Ceiling-Ground)

Device Placement, by the Author

\begin{tabular}{|c|c|c|c|c|c|c|c|}
\hline \multicolumn{2}{|c|}{ Device Location } & \multirow{2}{*}{$\begin{array}{l}\text { Activity } \\
\text { Location }\end{array}$} & \multirow{2}{*}{$\begin{array}{c}\text { Body } \\
\text { Orientation }\end{array}$} & \multicolumn{3}{|c|}{$\begin{array}{c}\text { Number of Samples per } \\
\text { Sit-Stand Style/Speed }\end{array}$} & \multirow[t]{2}{*}{ Total } \\
\hline $\mathbf{A P}$ & STA & & & 1 & 2 & 3 & \\
\hline \multirow{6}{*}{ Ceiling } & \multirow{6}{*}{ Ground } & \multirow{6}{*}{ Middle } & Front & 1 & 1 & 1 & 3 \\
\hline & & & Back & 1 & 1 & 1 & 3 \\
\hline & & & Towards AP & 1 & 1 & 1 & 3 \\
\hline & & & Towards STA & 1 & 1 & 1 & 3 \\
\hline & & & Left & 1 & 1 & 1 & 3 \\
\hline & & & Right & 1 & 1 & 1 & 3 \\
\hline \multirow{6}{*}{ Ceiling } & \multirow{6}{*}{ Ground } & \multirow{6}{*}{ Near AP } & Front & 1 & 1 & 1 & 3 \\
\hline & & & Back & 1 & 1 & 1 & 3 \\
\hline & & & Towards AP & 1 & 1 & 1 & 3 \\
\hline & & & Towards STA & 1 & 1 & 1 & 3 \\
\hline & & & Left & 1 & 1 & 1 & 3 \\
\hline & & & Right & 1 & 1 & 1 & 3 \\
\hline \multirow{6}{*}{ Ceiling } & \multirow{6}{*}{ Ground } & \multirow{6}{*}{ Near STA } & Front & 1 & 1 & 1 & 3 \\
\hline & & & Back & 1 & 1 & 1 & 3 \\
\hline & & & Towards AP & 1 & 1 & 1 & 3 \\
\hline & & & Towards STA & 1 & 1 & 1 & 3 \\
\hline & & & Left & 1 & 1 & 1 & 3 \\
\hline & & & Right & 1 & 1 & 1 & 3 \\
\hline \multirow{4}{*}{ Ceiling } & \multirow{4}{*}{ Ground } & \multirow{4}{*}{$\begin{array}{l}\text { Front-Left } \\
\text { Corner }\end{array}$} & Front & 1 & 1 & 1 & 3 \\
\hline & & & Back & 1 & 1 & 1 & 3 \\
\hline & & & Left & 1 & 1 & 1 & 3 \\
\hline & & & Right & 1 & 1 & 1 & 3 \\
\hline \multirow{4}{*}{ Ceiling } & \multirow{4}{*}{ Ground } & \multirow{4}{*}{$\begin{array}{l}\text { Back-Right } \\
\text { Corner }\end{array}$} & Front & 1 & 1 & 1 & 3 \\
\hline & & & Back & 1 & 1 & 1 & 3 \\
\hline & & & Left & 1 & 1 & 1 & 3 \\
\hline & & & Right & 1 & 1 & 1 & 3 \\
\hline \multicolumn{4}{|c|}{ TOTAL NUMBER OF DATA SAMPLES } & 26 & 26 & 26 & 78 \\
\hline
\end{tabular}

Table 39 Summary of Sitting and Standing Data Samples Collected by Three Volunteer Subjects

\begin{tabular}{|c|c|c|c|c|c|c|c|}
\hline \multirow{2}{*}{ Device Location } & \multirow{2}{*}{$\begin{array}{c}\text { Activity } \\
\text { Location }\end{array}$} & \multirow{2}{*}{$\begin{array}{c}\text { Body } \\
\text { Orientation }\end{array}$} & \multicolumn{3}{|c|}{$\begin{array}{c}\text { Number of Samples } \\
\text { by Subject }\end{array}$} & \multirow{2}{*}{ Total } \\
\cline { 1 - 4 }$n n$ & AP & STA & & M & J & H & \\
\hline Ceiling & Ground & Random & Random & 2 & 2 & 3 & 7 \\
\hline
\end{tabular}


Table 40 Summary of Additional Data Samples Collected by the Author, but Not Used

\begin{tabular}{|c|c|c|c|c|c|c|}
\hline \multicolumn{2}{|c|}{ Device Location } & \multirow{2}{*}{$\begin{array}{l}\text { Activity/Chair } \\
\text { Location }\end{array}$} & \multirow{2}{*}{$\begin{array}{c}\text { Body } \\
\text { Orientation }\end{array}$} & \multirow{2}{*}{ Activity } & \multirow{2}{*}{$\begin{array}{c}\text { Number } \\
\text { of } \\
\text { Samples }\end{array}$} & \multirow{2}{*}{ Total } \\
\hline $\mathbf{A P}$ & STA & & & & & \\
\hline \multirow{7}{*}{ Ground } & \multirow{7}{*}{ Ground } & Middle & Front/Back/Left & Seated & 6 & 6 \\
\hline & & Middle & Front/Back/Left & Standstill & 6 & 6 \\
\hline & & Middle & $\mathrm{N} / \mathrm{A}$ & \multirow{4}{*}{$\begin{array}{l}\text { Empty } \\
\text { Room }\end{array}$} & 3 & \multirow{4}{*}{6} \\
\hline & & Near Receiver & $\mathrm{N} / \mathrm{A}$ & & 1 & \\
\hline & & $\begin{array}{l}\text { Front-Left } \\
\text { Corner }\end{array}$ & N/A & & 1 & \\
\hline & & $\begin{array}{c}\text { Back-Right } \\
\text { Corner }\end{array}$ & $\mathrm{N} / \mathrm{A}$ & & 1 & \\
\hline & & Middle & Random & $\begin{array}{l}\text { Walking } \\
\text { back and } \\
\text { fourth } \\
\text { across the } \\
\text { room }\end{array}$ & 3 & 3 \\
\hline \multirow{7}{*}{ Ceiling } & \multirow{7}{*}{ Ground } & Middle & Front/Back/Left & Seated & 1 & 1 \\
\hline & & Middle & Front/Back/Left & Standstill & 1 & 1 \\
\hline & & Middle & $\mathrm{N} / \mathrm{A}$ & \multirow{5}{*}{$\begin{array}{l}\text { Empty } \\
\text { Room }\end{array}$} & 1 & \multirow{5}{*}{5} \\
\hline & & $\begin{array}{c}\text { Near } \\
\text { Transmitter }\end{array}$ & N/A & & 1 & \\
\hline & & Near Receiver & $\mathrm{N} / \mathrm{A}$ & & 1 & \\
\hline & & $\begin{array}{c}\text { Front-Left } \\
\text { Corner }\end{array}$ & N/A & & 1 & \\
\hline & & $\begin{array}{c}\text { Back-Right } \\
\text { Corner }\end{array}$ & N/A & & 1 & \\
\hline \multicolumn{6}{|c|}{ TOTAL NUMBER OF DATA SAMPLES } & 28 \\
\hline
\end{tabular}




\section{Appendix B Samples of Preprocessed Data}

This appendix includes figures displaying and comparing samples of the data produced by different processing techniques. This appendix is to presents how the pattern in one CSI capture changes depending on the applied preprocessing technique(s). Although the evident pattern in sole preprocessing methods, containing a singly preprocessing technique (filtering, normalization, or feature scaling) was destroyed when applying a combination of methods (see Figure 23 in Appendix B.5), the combined preprocessing approach optimized the classification performance.

\section{B.1 Raw Signal}

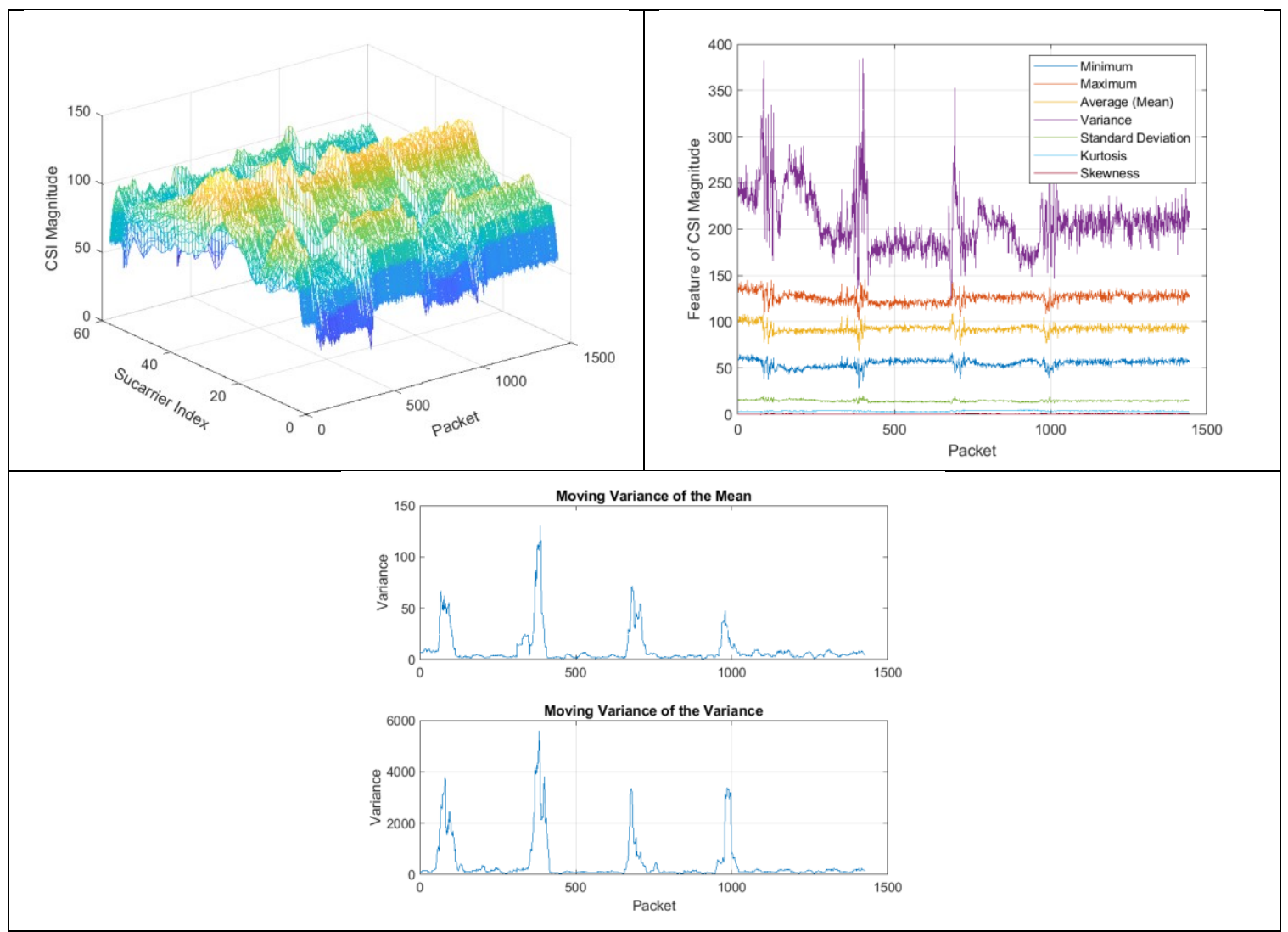

Figure 14 Sample Plots of a Raw Signal 


\section{B.2 Filtering}

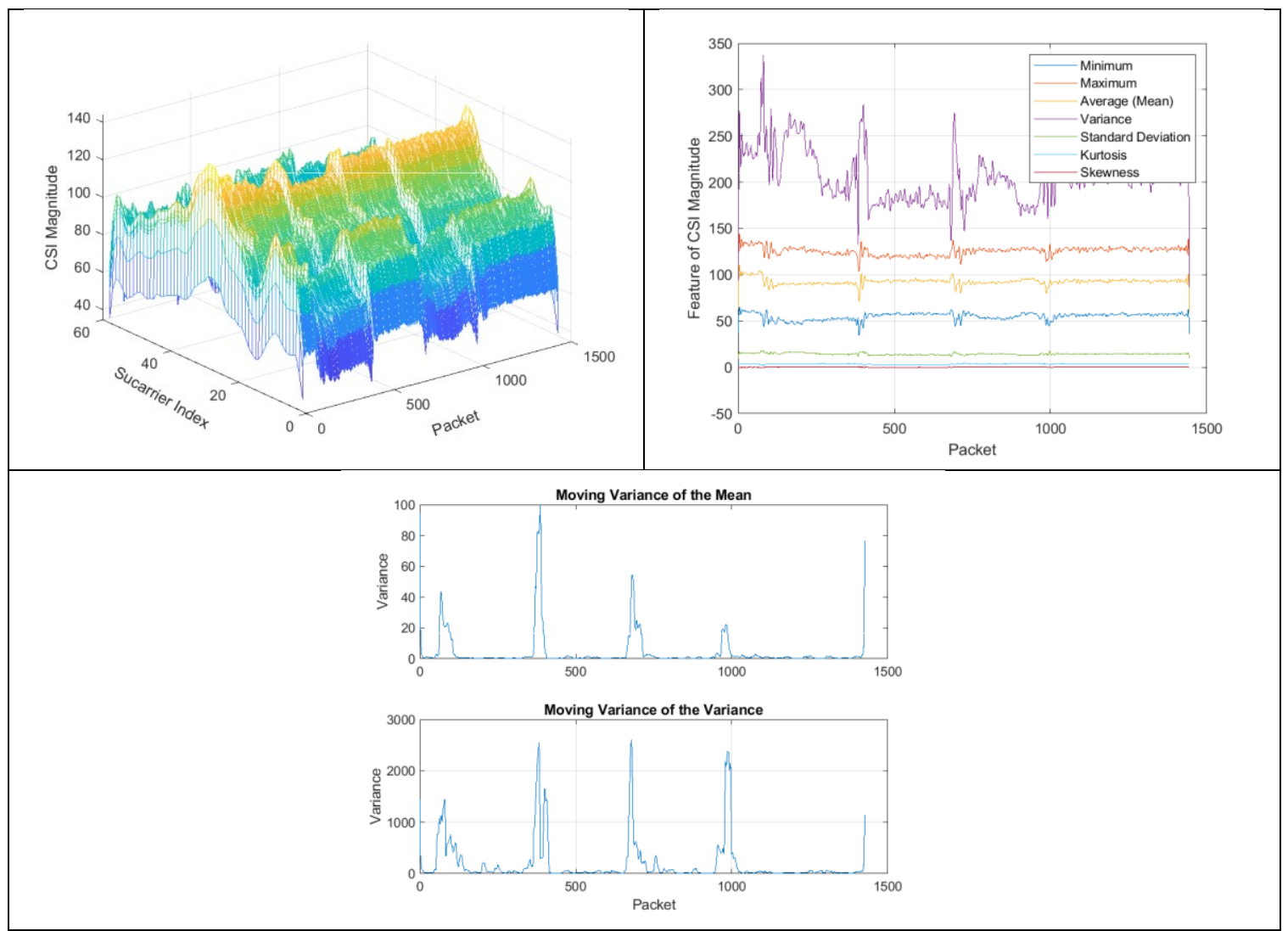

Figure 15 Sample Plots of a Filtered Signal 


\section{B.3 Normalization}

\section{Z-Score Standardization}

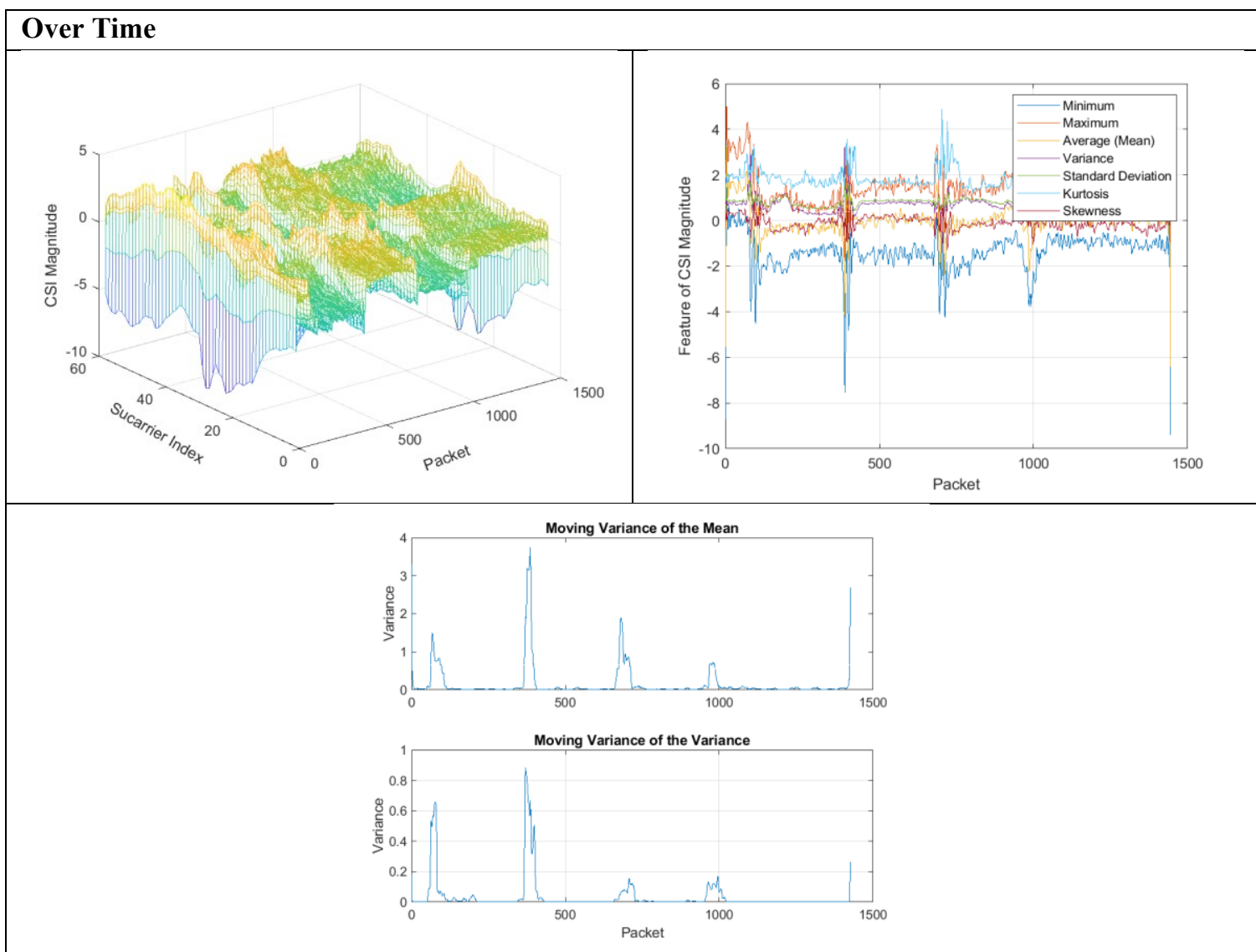

Figure 16 Sample Plots of Signals Normalized via Z-Score Standardization, over the Time/Packet

Domain 

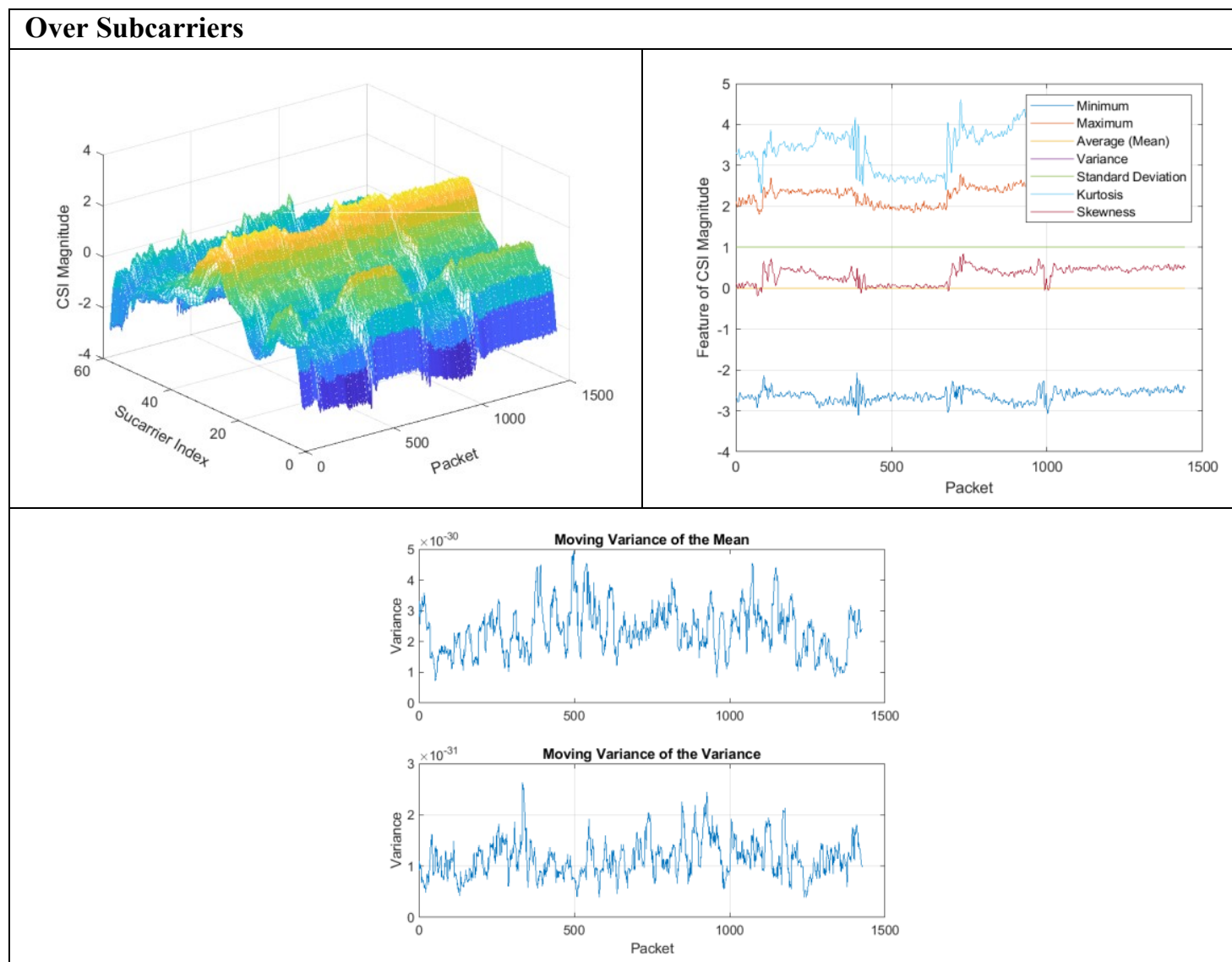

Figure 17 Sample Plots of Signals Normalized via Z-Score Standardization, over the Subcarriers Domain 


\section{Fixed-Factor Normalization}

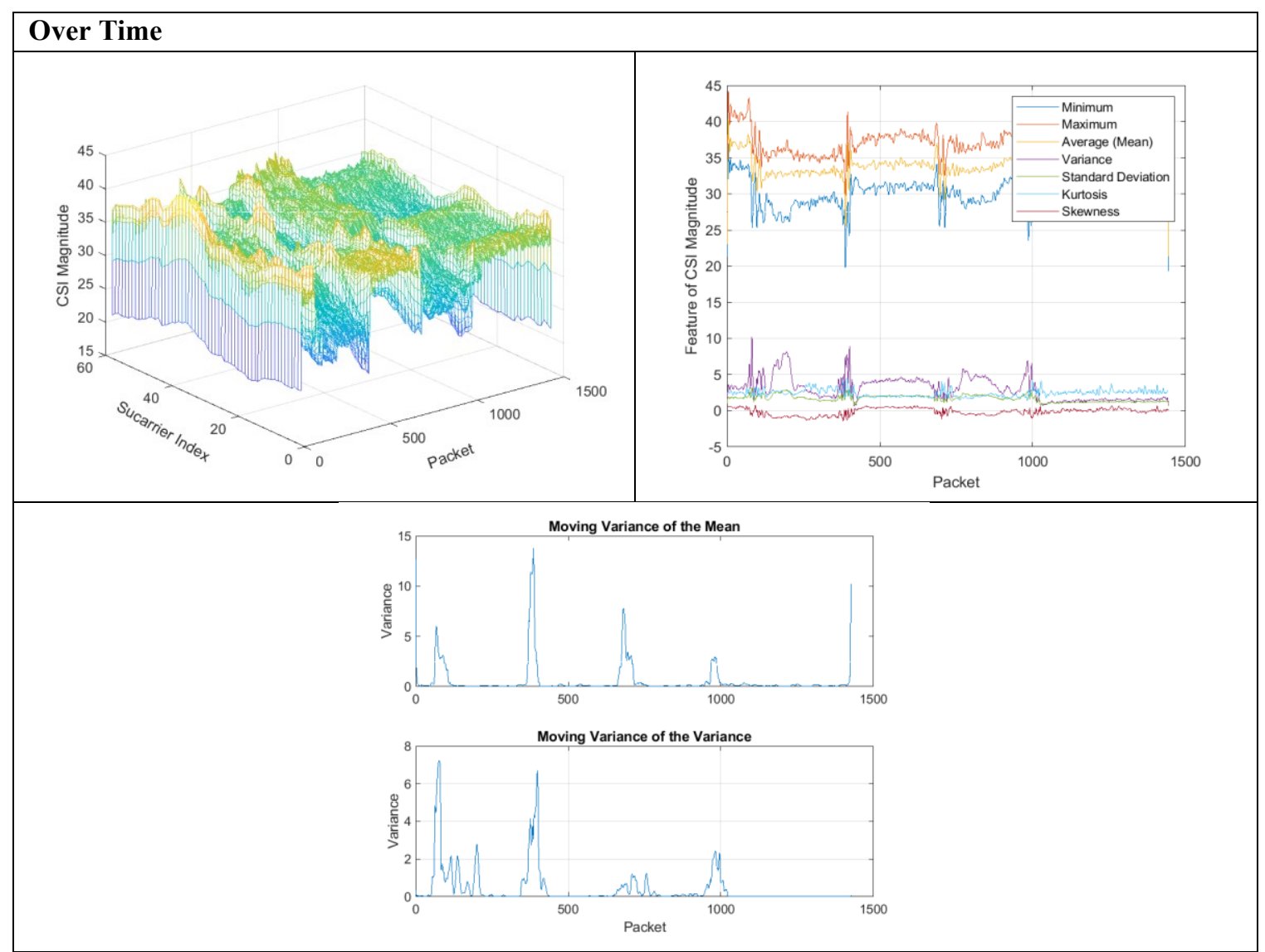

Figure 18 Sample Plots of Signals Normalized using a Fixed-Factor, over the Time/Packet Domain 


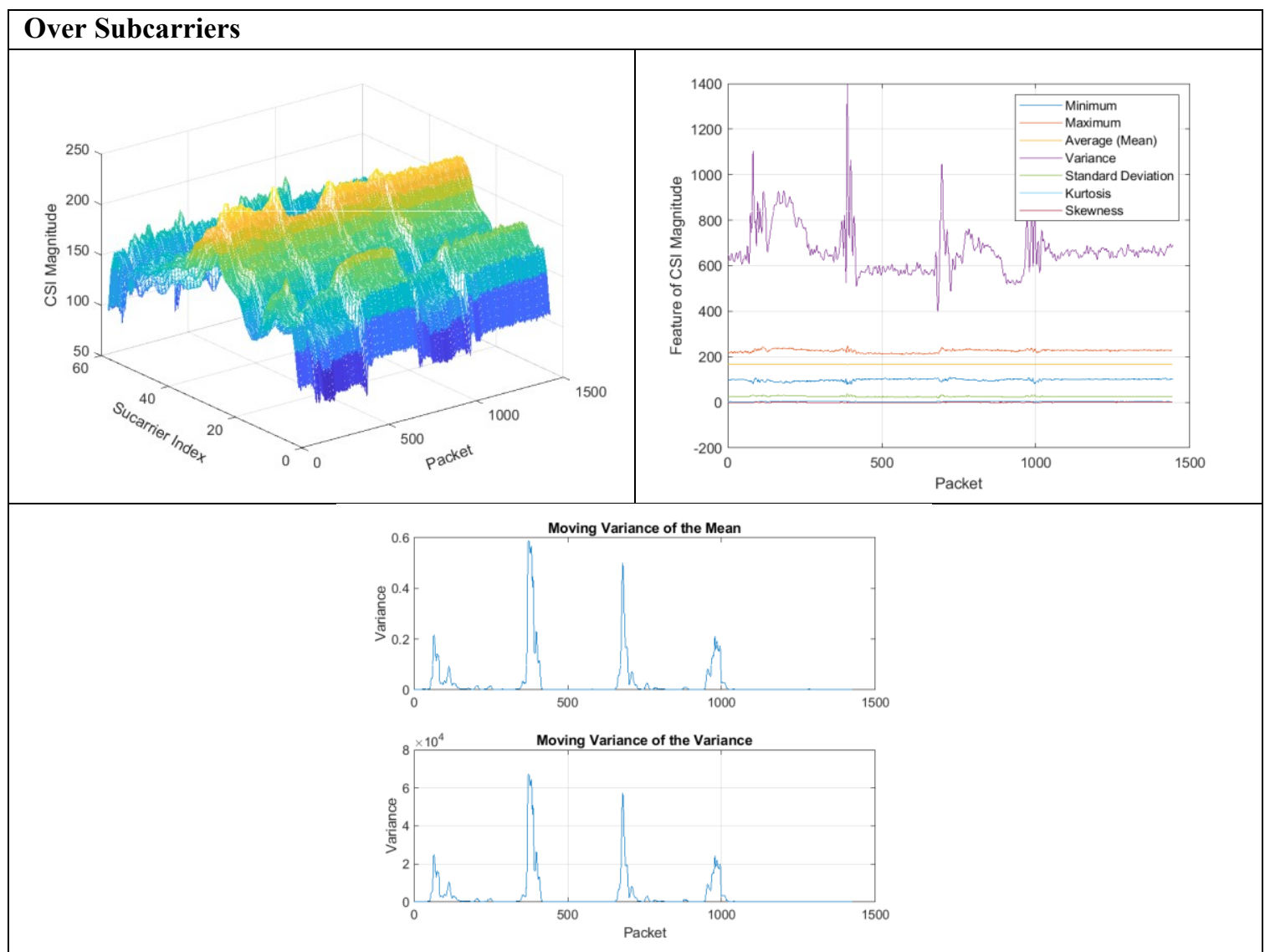

Figure 19 Sample Plots of Signals Normalized using a Fixed-Factor, over the Subcarriers Domain 


\section{B.4 Feature Scaling}

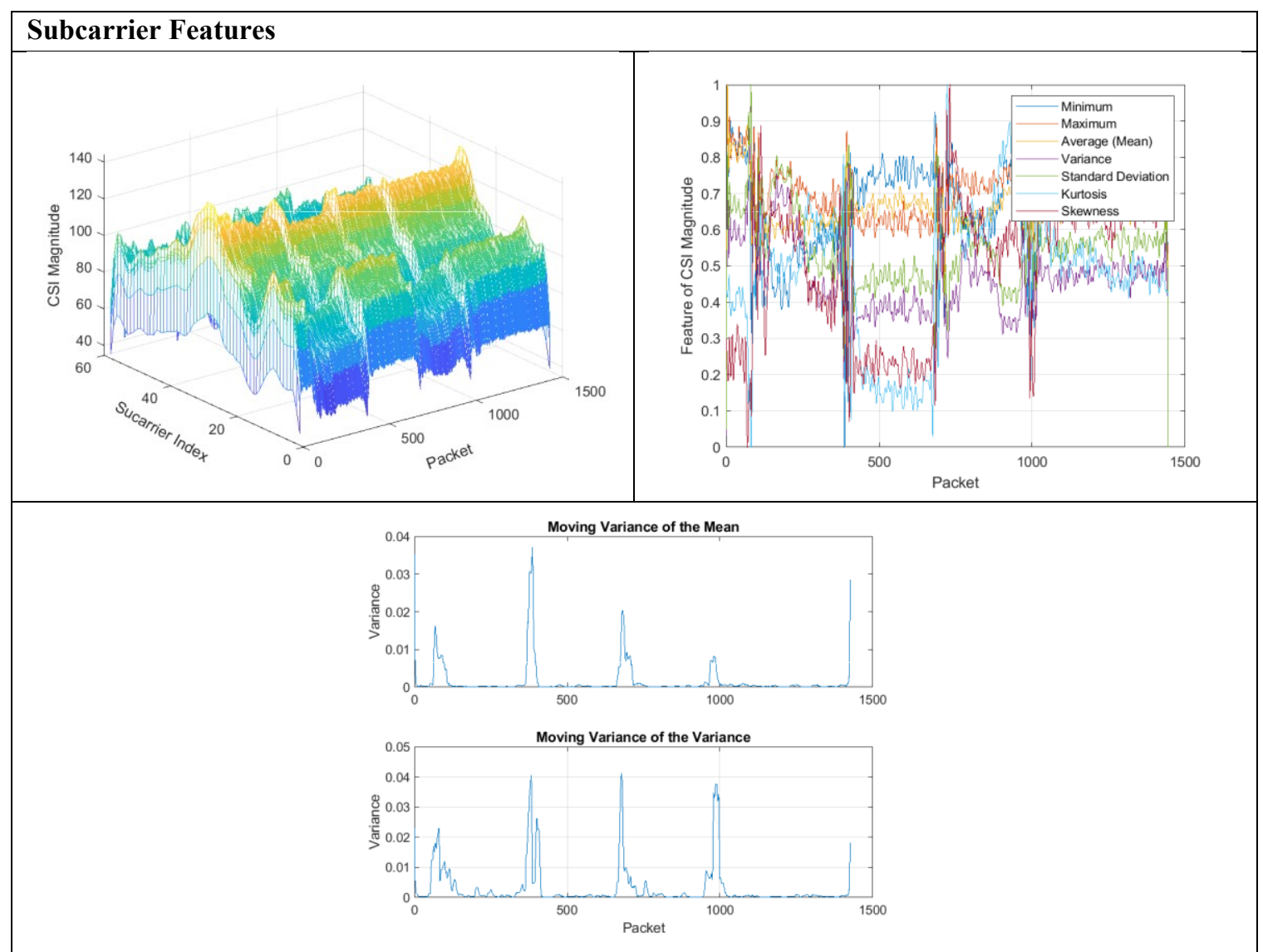

Figure 20 Sample Plots of Signals Scaled through Subcarrier Features 


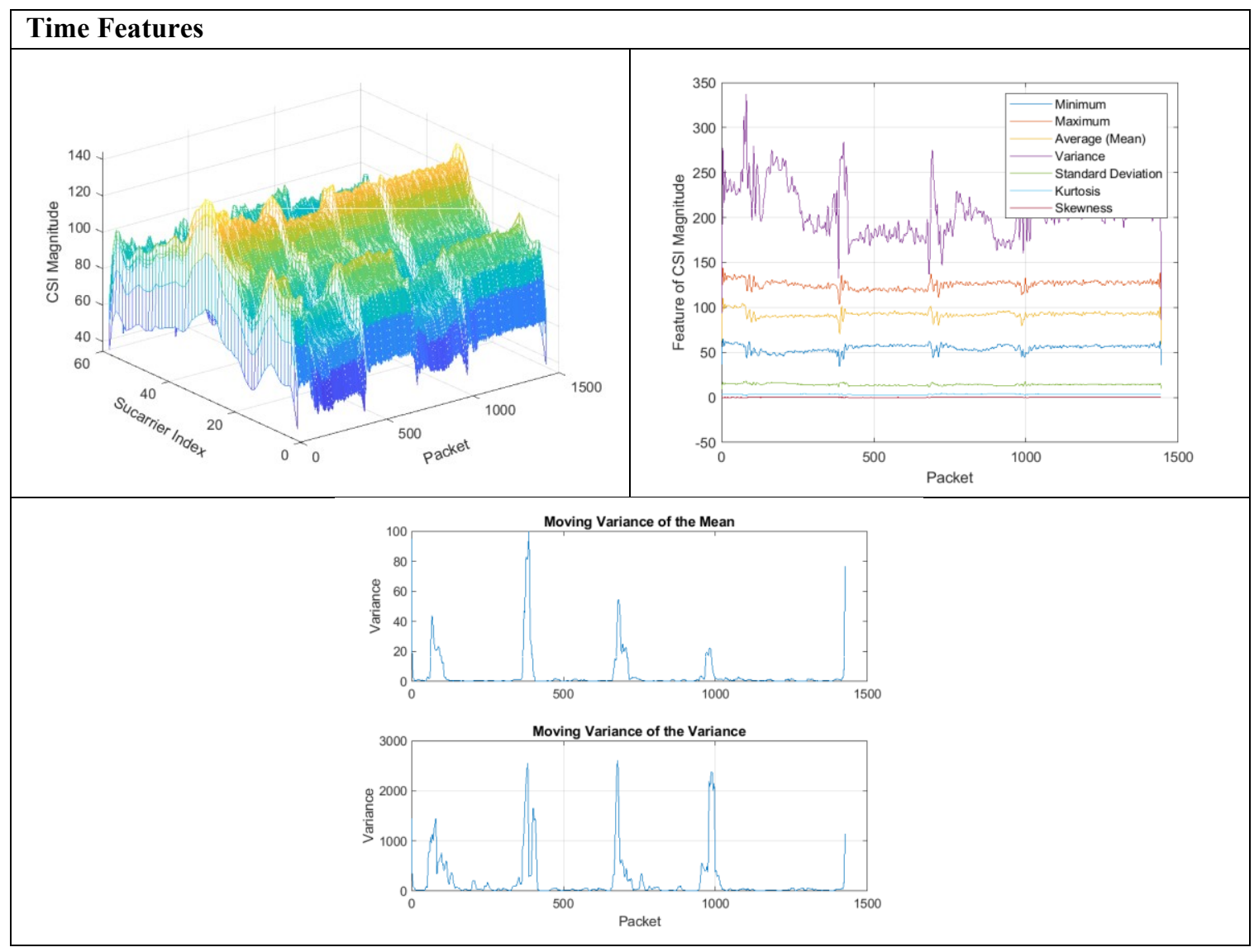

Figure 21 Sample Plots of Signals Scaled through Time Features 


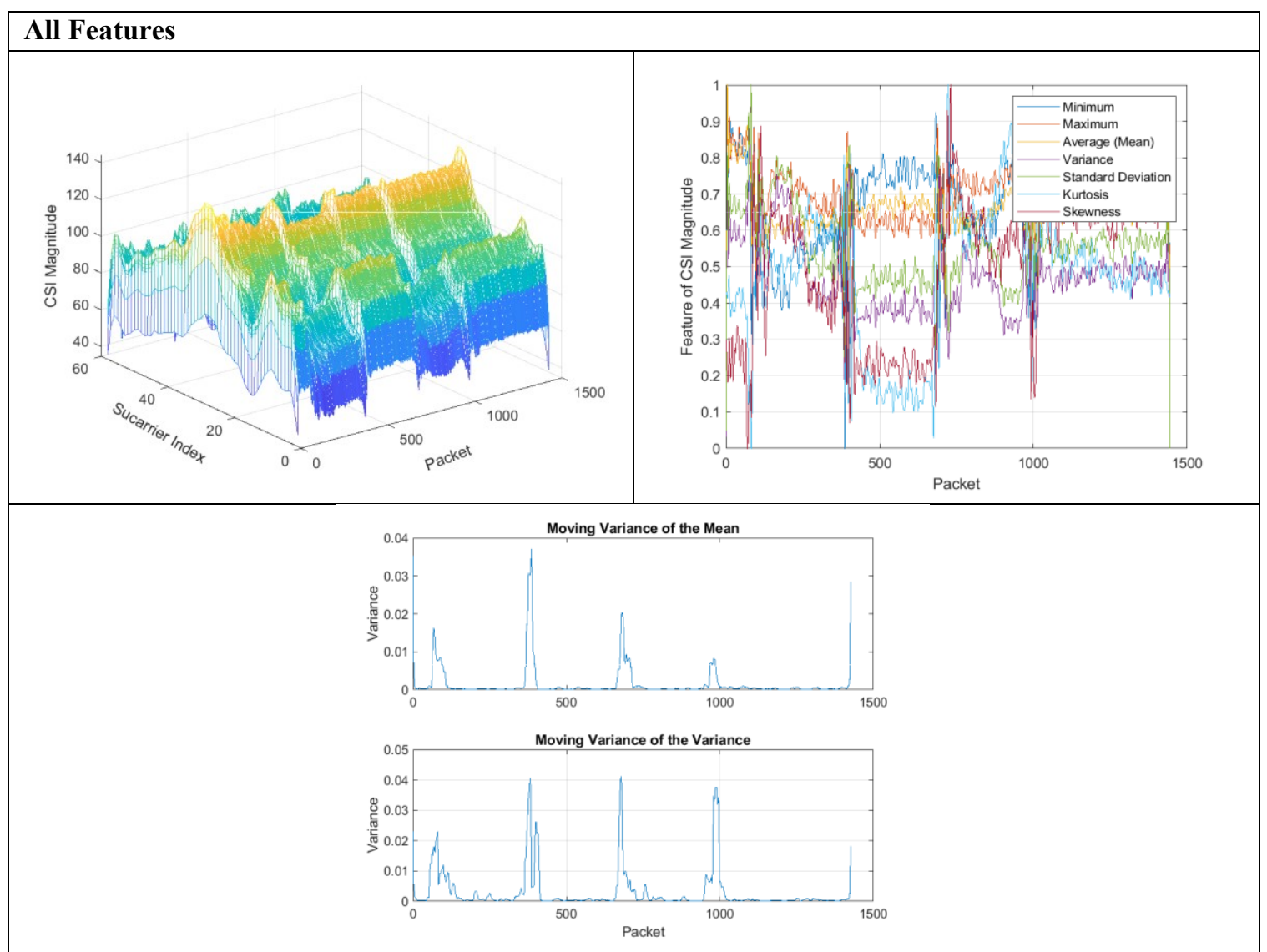

Figure 22 Sample Plots of Signals Scaled through Both Subcarrier and Time Features 


\section{B.5 Filtering, Z-Score Standardization, and Feature Scaling Combined}

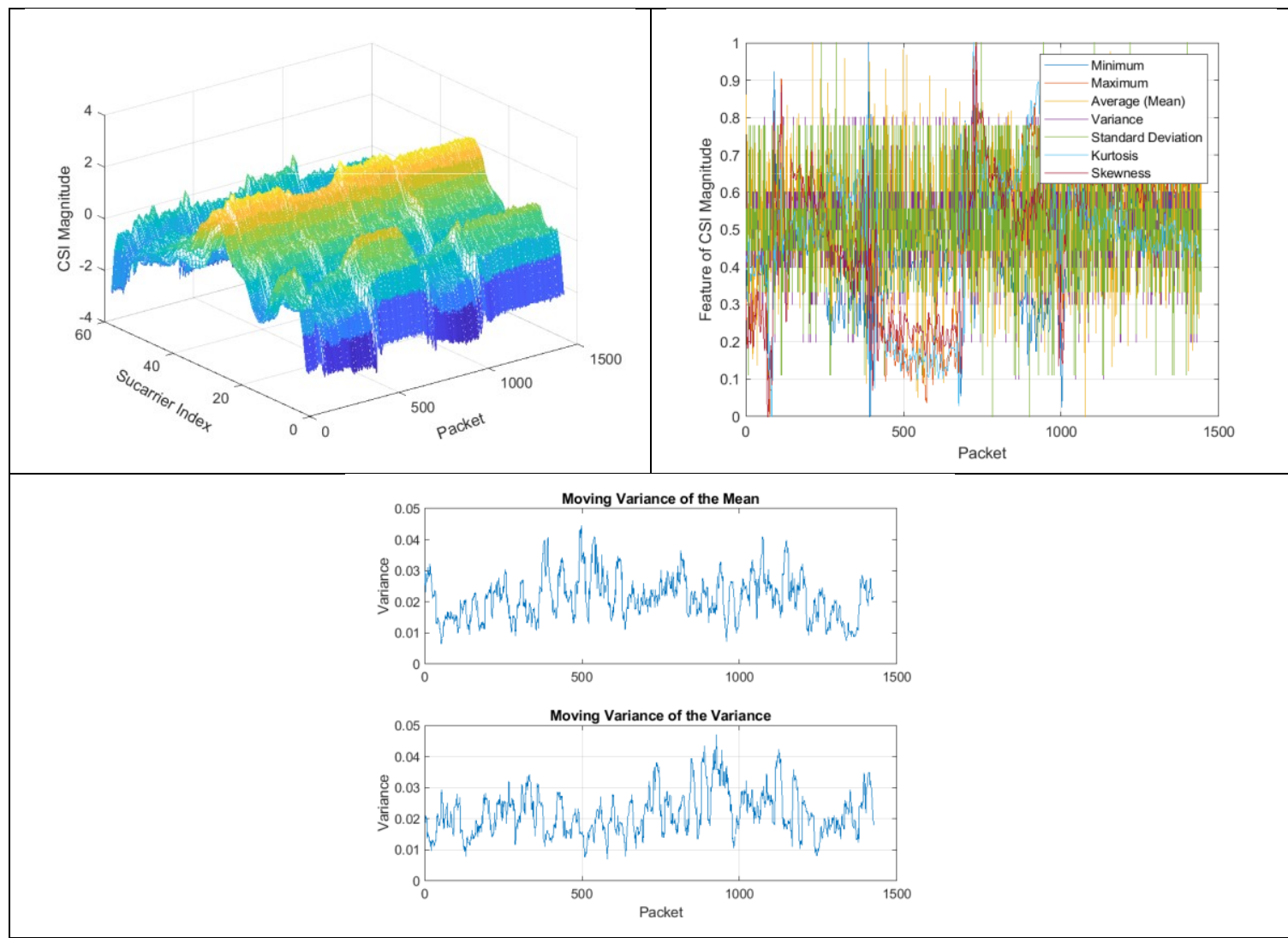

Figure 23 Sample Plots of Filtered, Z-Score Standardized (over subcarriers), and Scaled (all features) Signals 


\section{Appendix C Samples of Data from Different Activity Locations}

This appendix includes figures representing data samples collected at different locations around the laboratory.

\section{C.1 Middle}

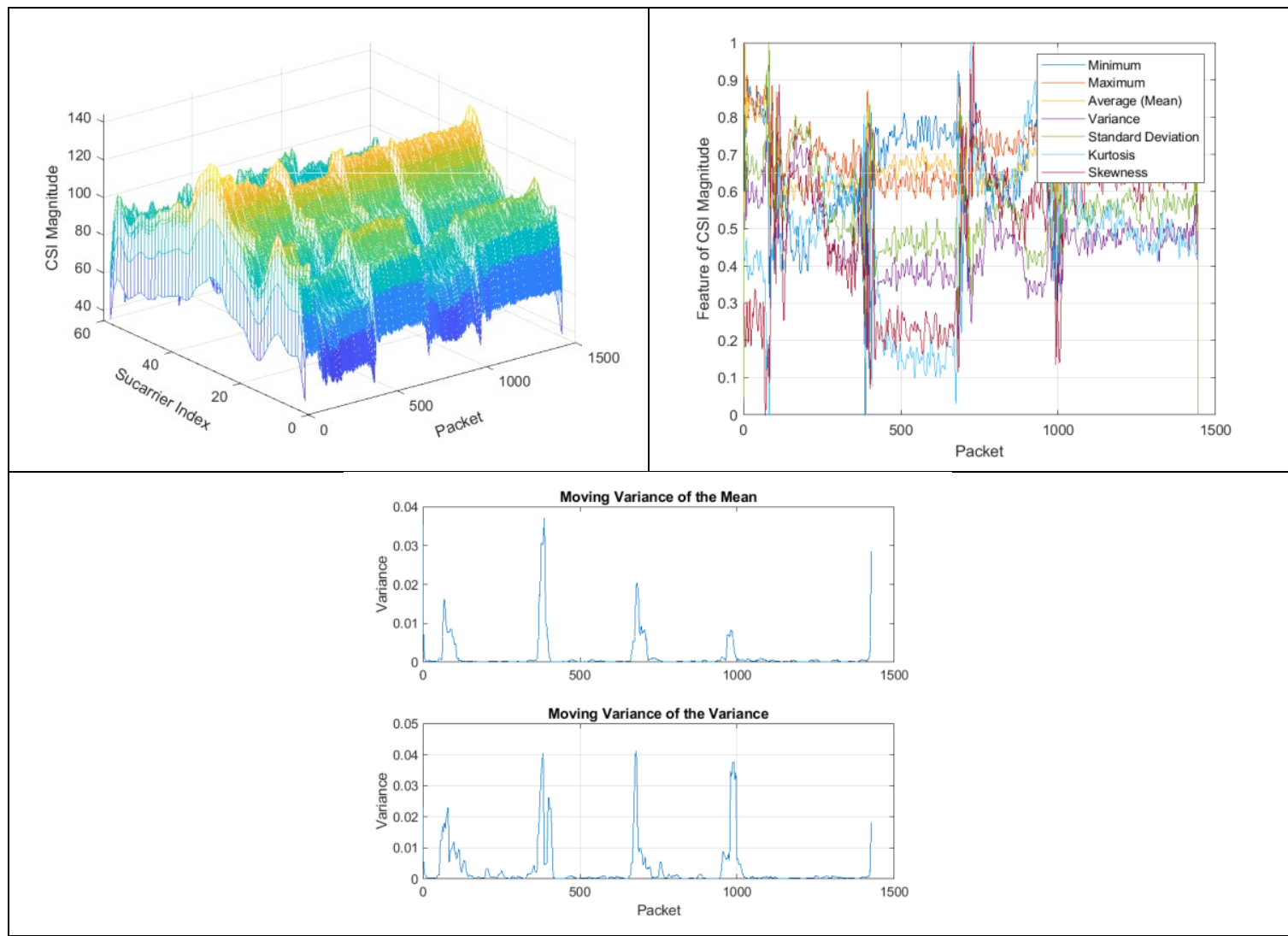

Figure 24 Sample Plots of Data Collected at the Middle of a Room 


\section{C.2 Near the Transmitter}

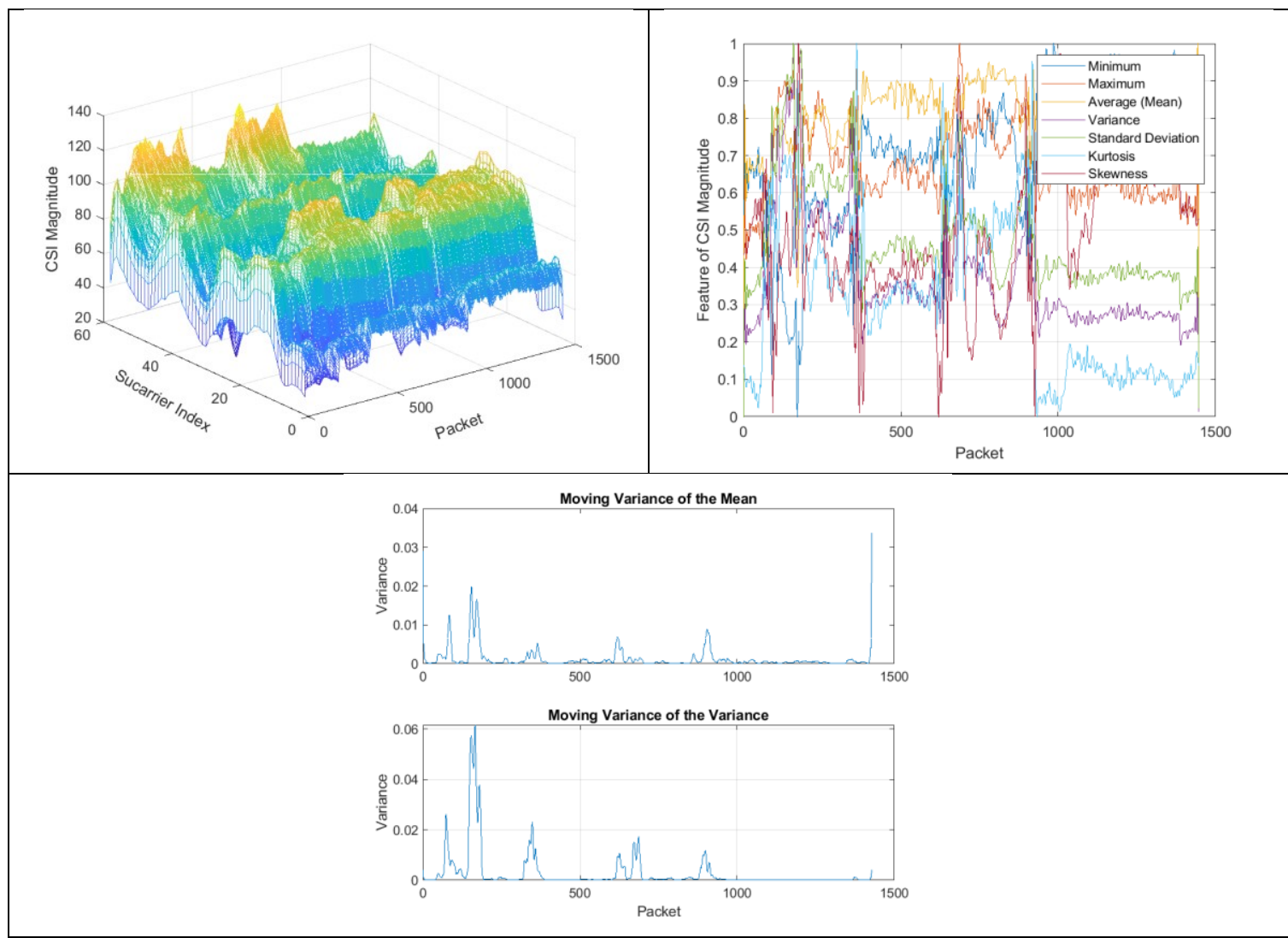

Figure 25 Sample Plots of Data Collected near the Transmitter 


\section{C.3 Near the Receiver}

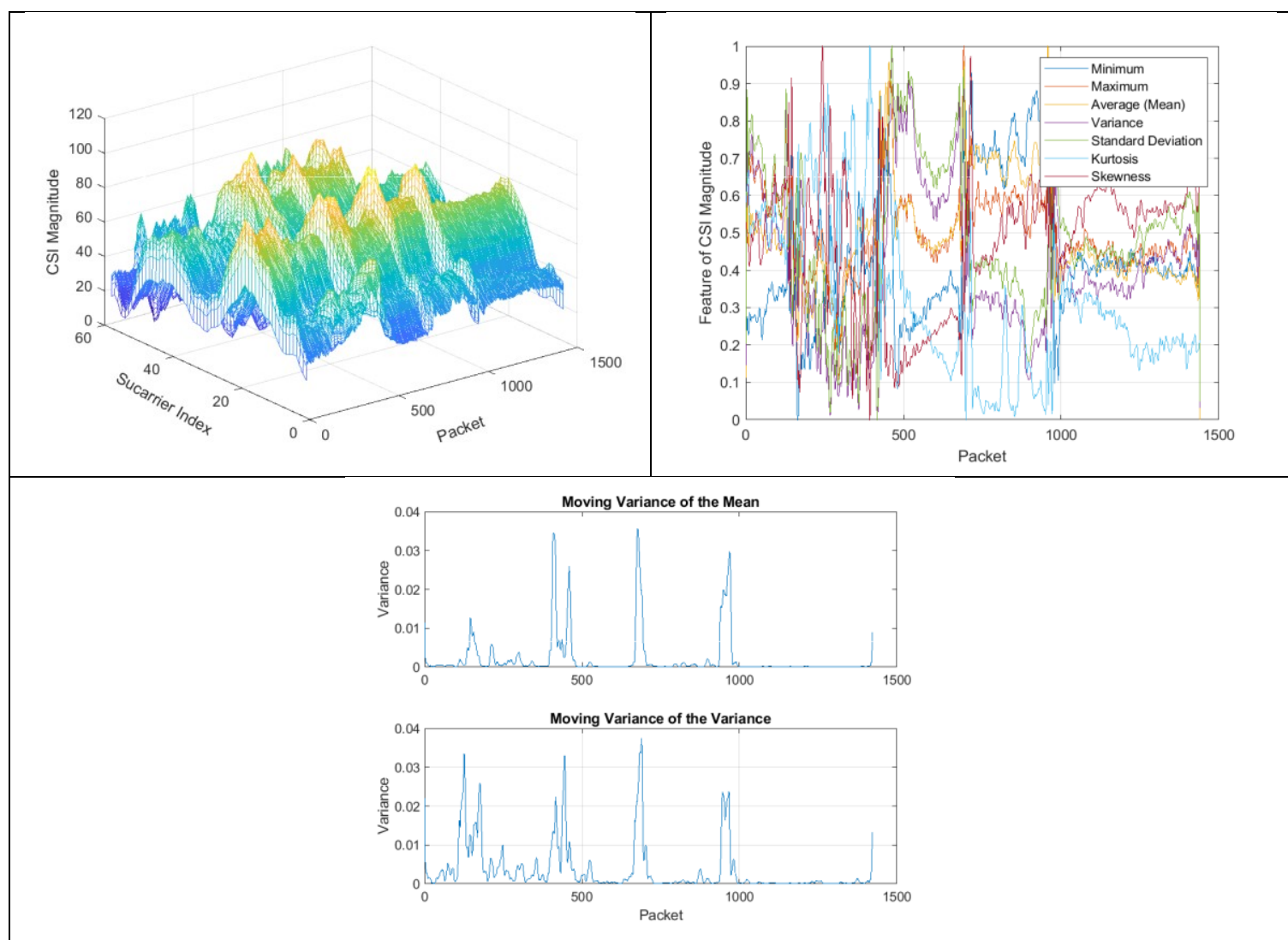

Figure 26 Sample Plots of Data Collected near the Receiver 


\section{C.4 Empty Corners}

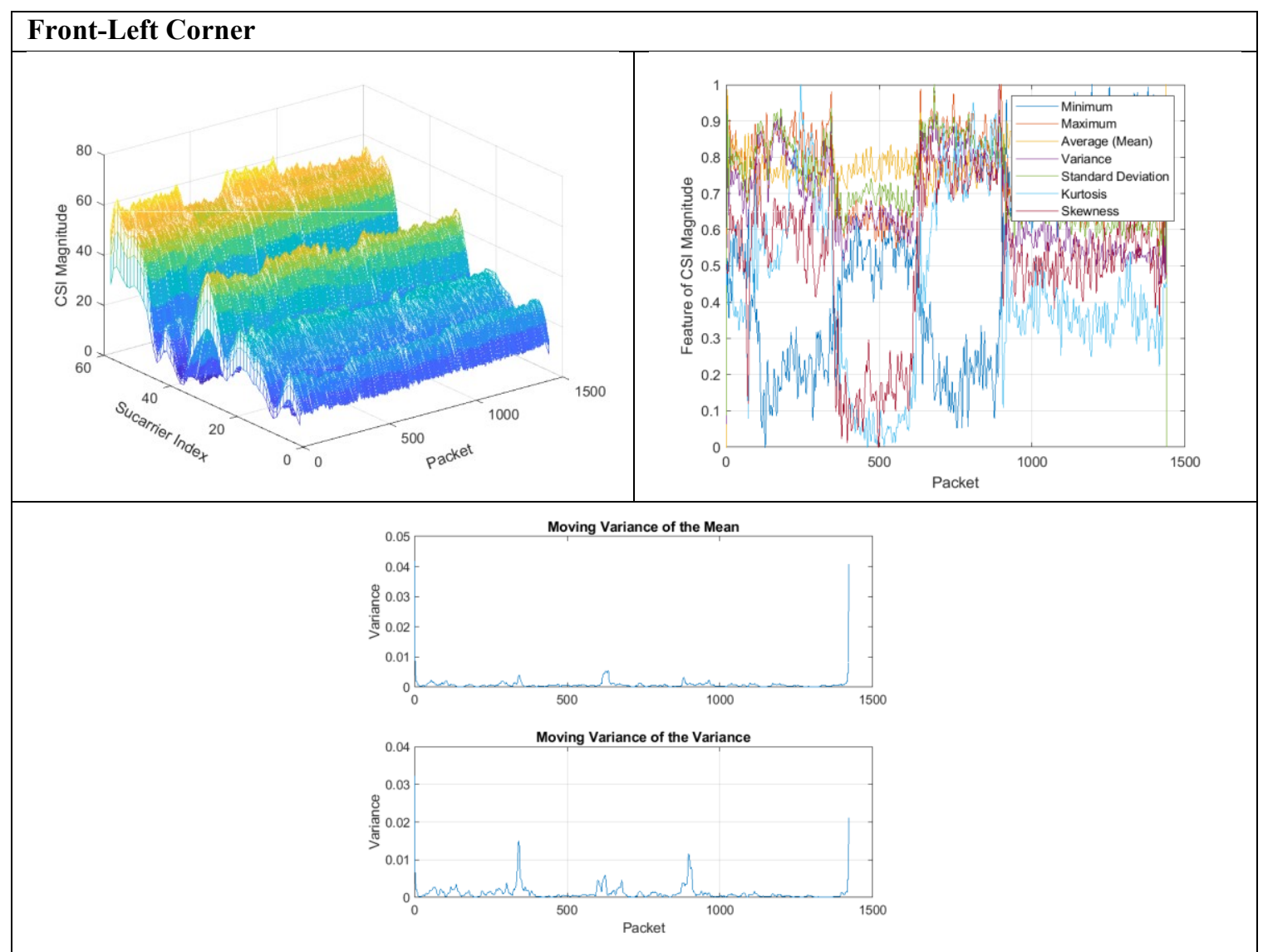

Figure 27 Sample of Plots of Data Collected at the Empty Front-Left Corner of a Room 


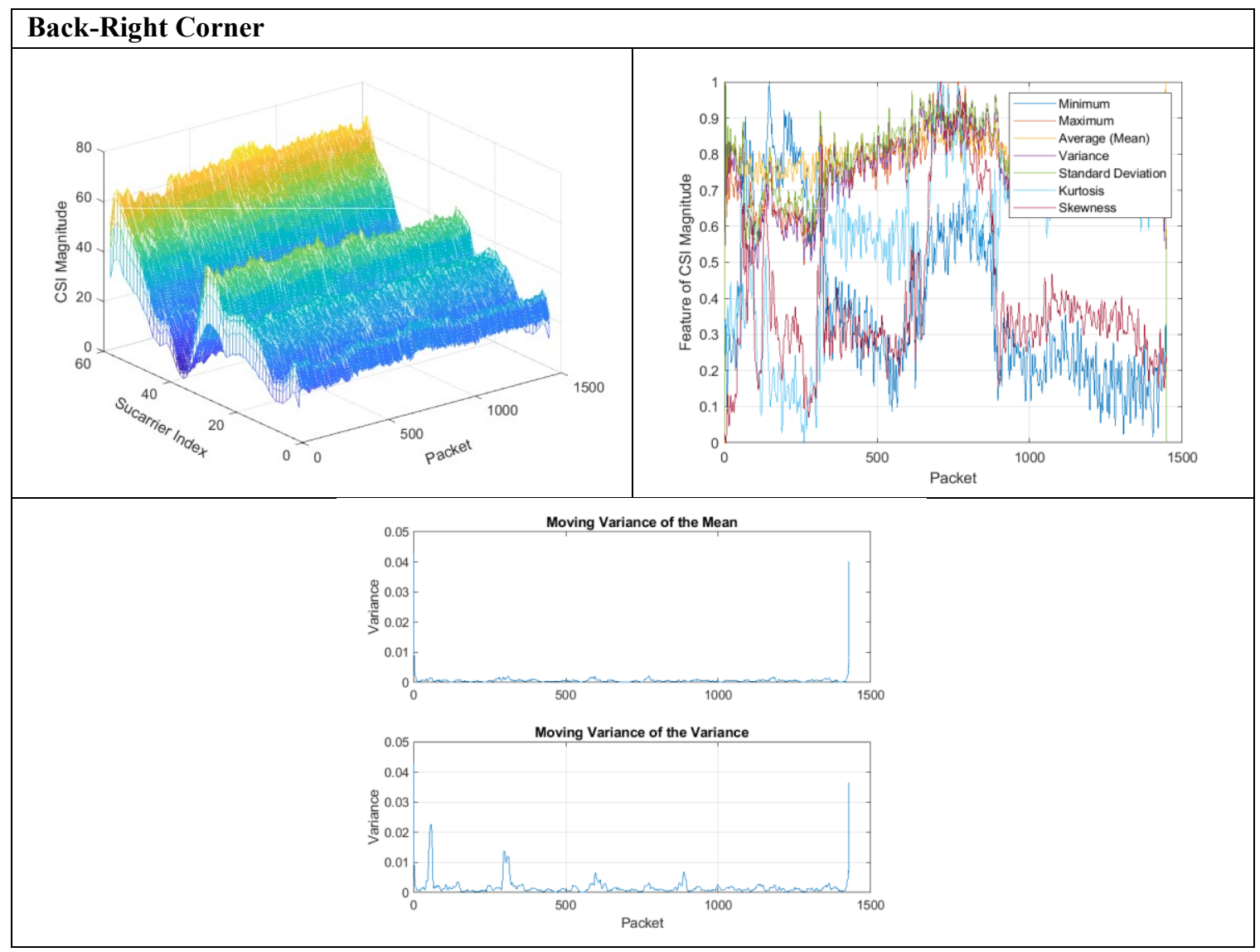

Figure 28 Sample of Plots of Data Collected at the Empty Back-Right Corner of a Room 


\section{Appendix D Samples of Data with Different Device Placements}

This appendix includes figures displaying data samples collected with differing device placements.

\section{D.1 2D (Ground-Ground) Device Placement}

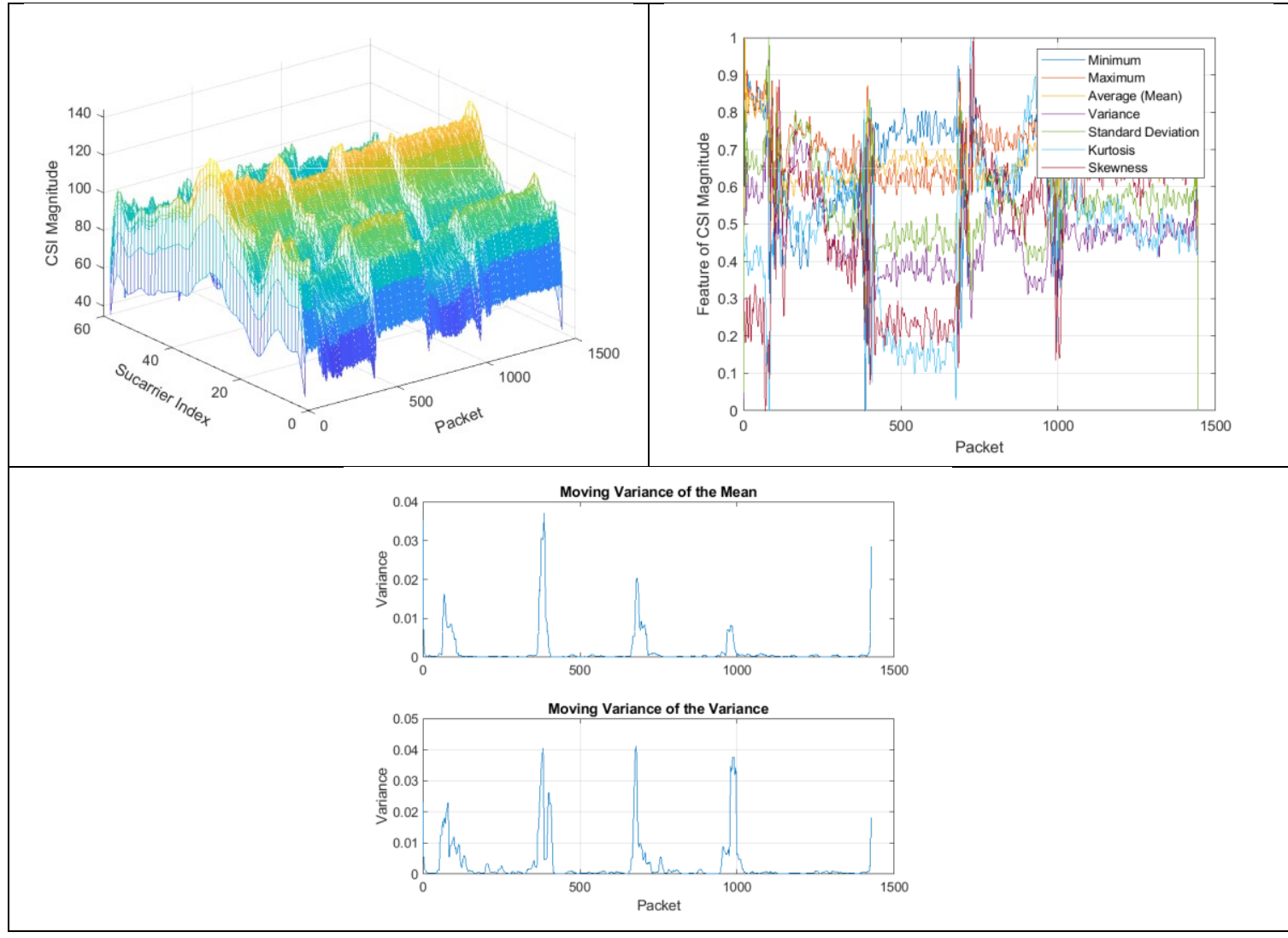

Figure 29 Sample of Plots with Data Collected under a 2D (Ground) Device Placement 


\section{D.2 3D (Ceiling-Ground) Device Placement}

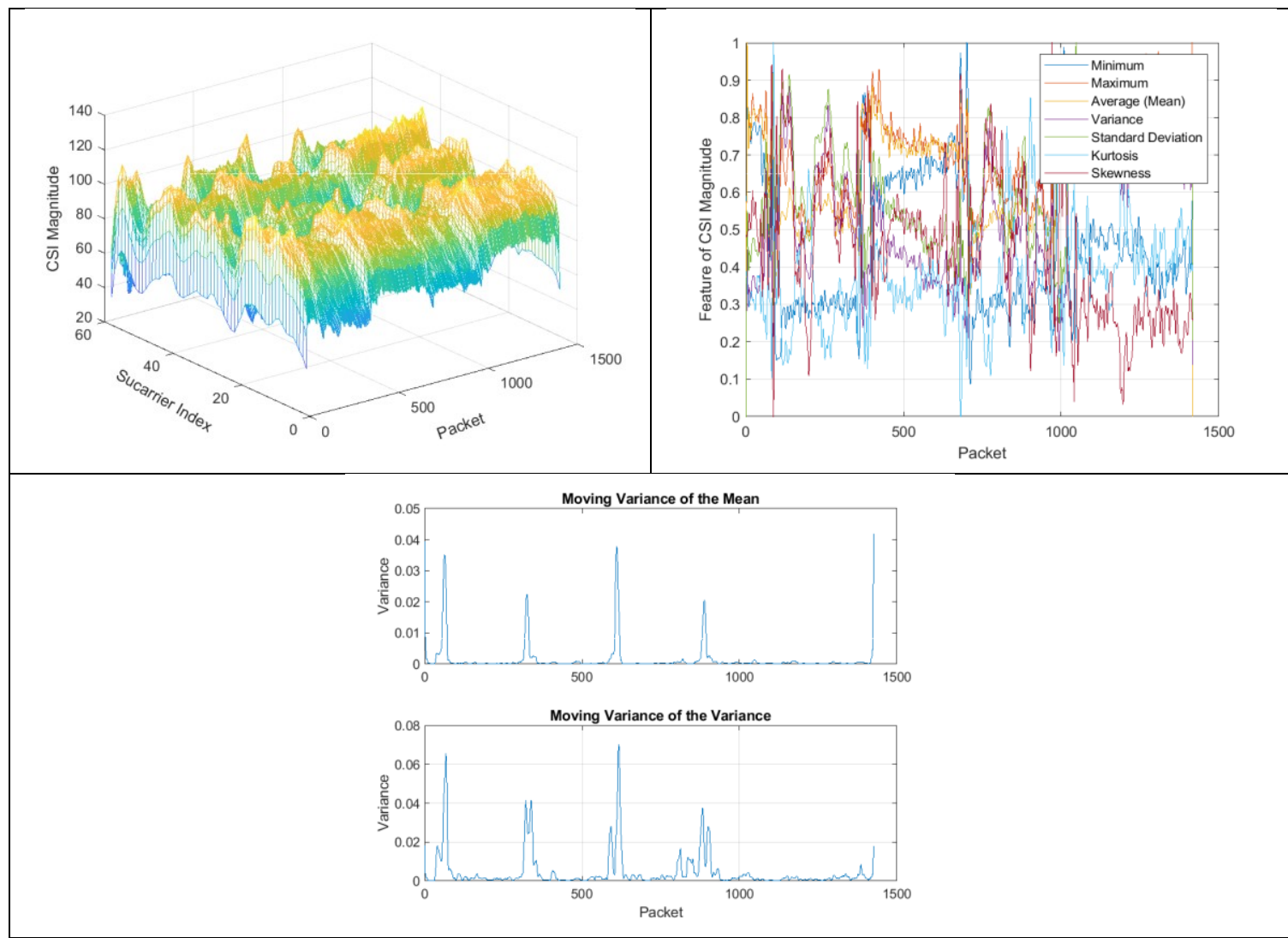

Figure 30 Sample of Plots with Data Collected under a 3D (Ground-Ceiling) Device Placement 


\section{Appendix E Samples of Data by Different Subjects}

This appendix includes figures displaying data samples collected by four different subjects.

\section{E.1 Subject 1 (I)}

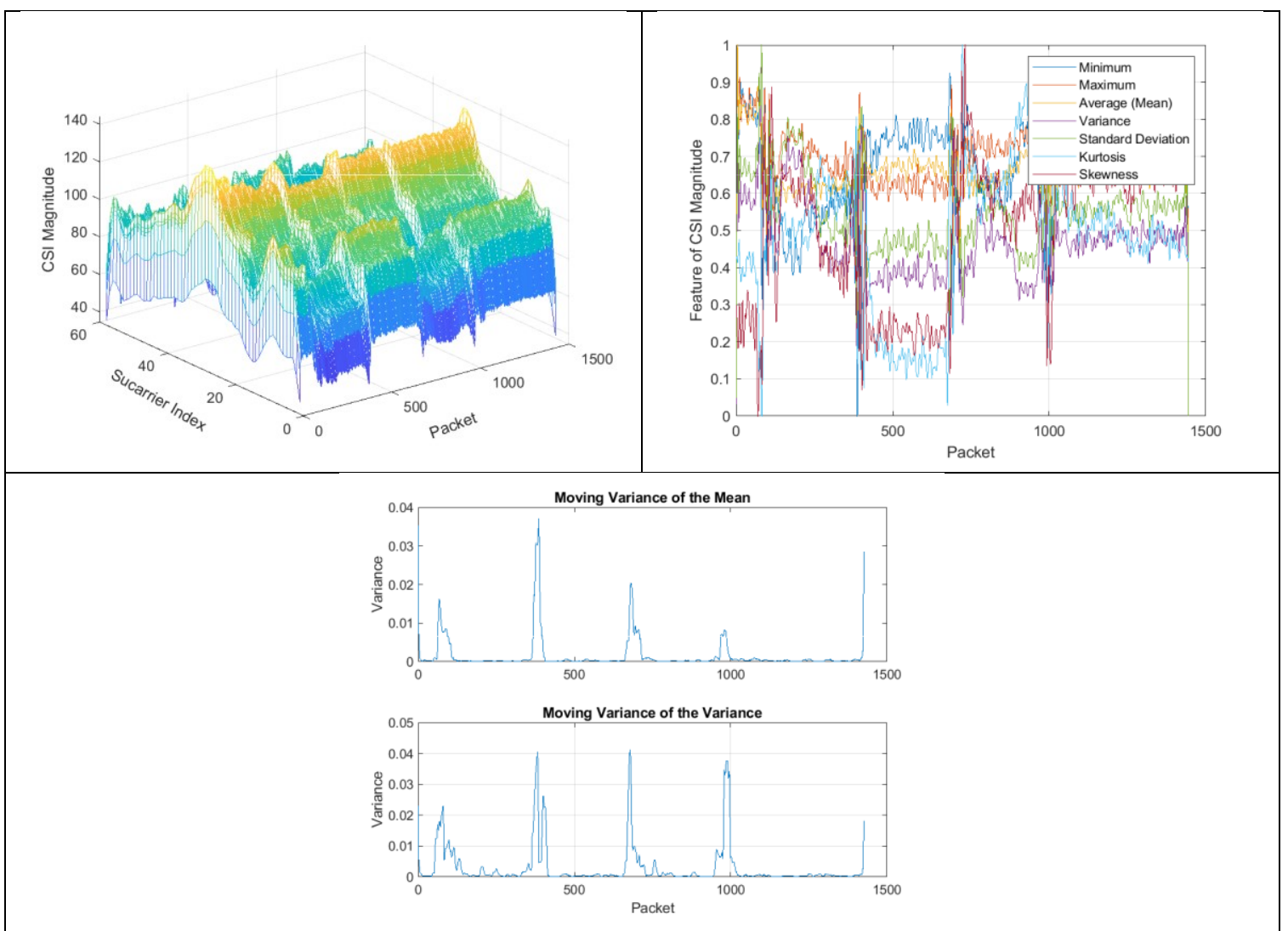

Figure 31 Sample of Plots for Data Collected by Subject 1 


\section{E.2 Subject 2 (M)}

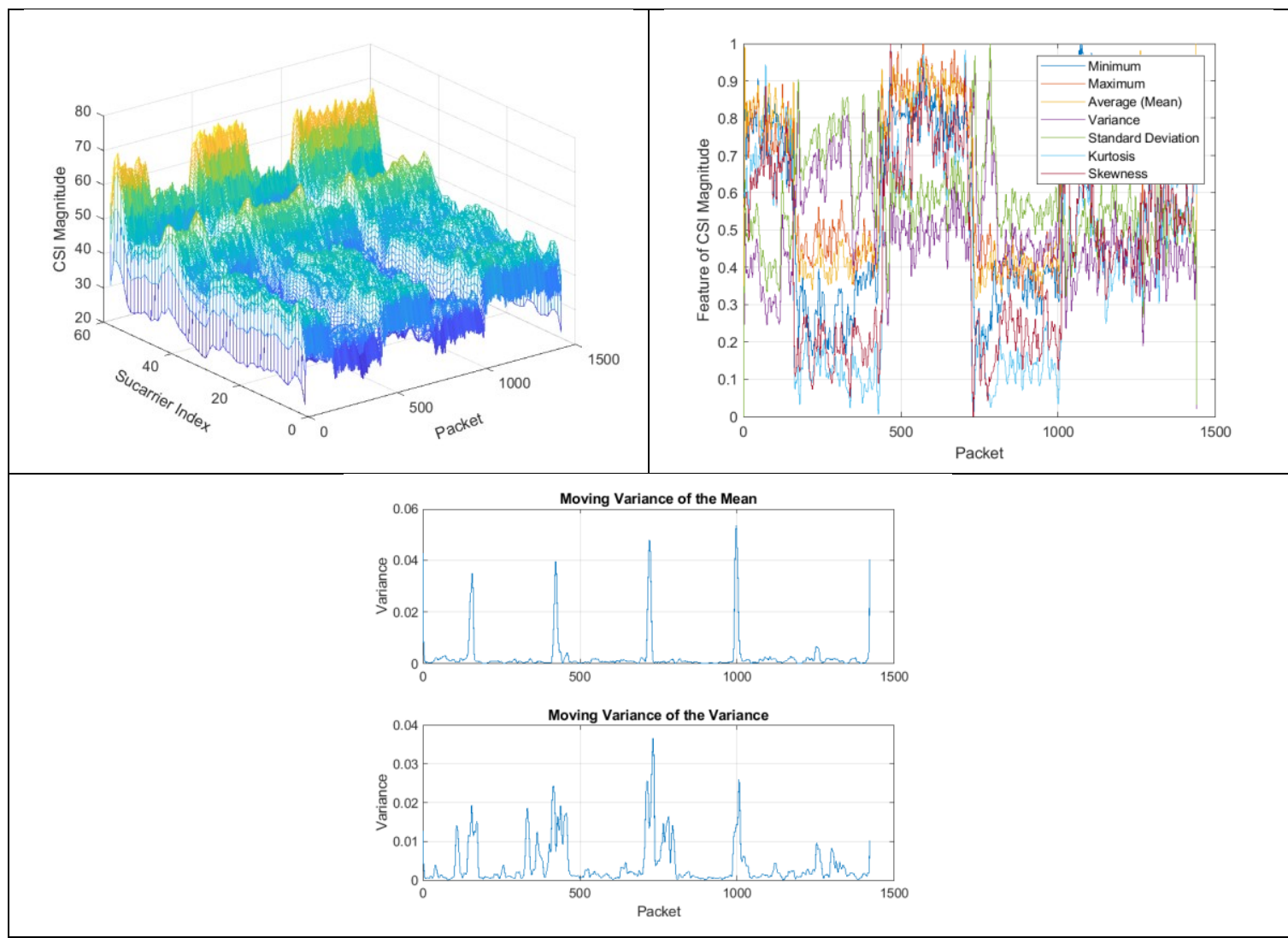

Figure 32 Sample of Plots for Data Collected by Subject 2 


\section{E.3 Subject $3(\mathbf{J})$}

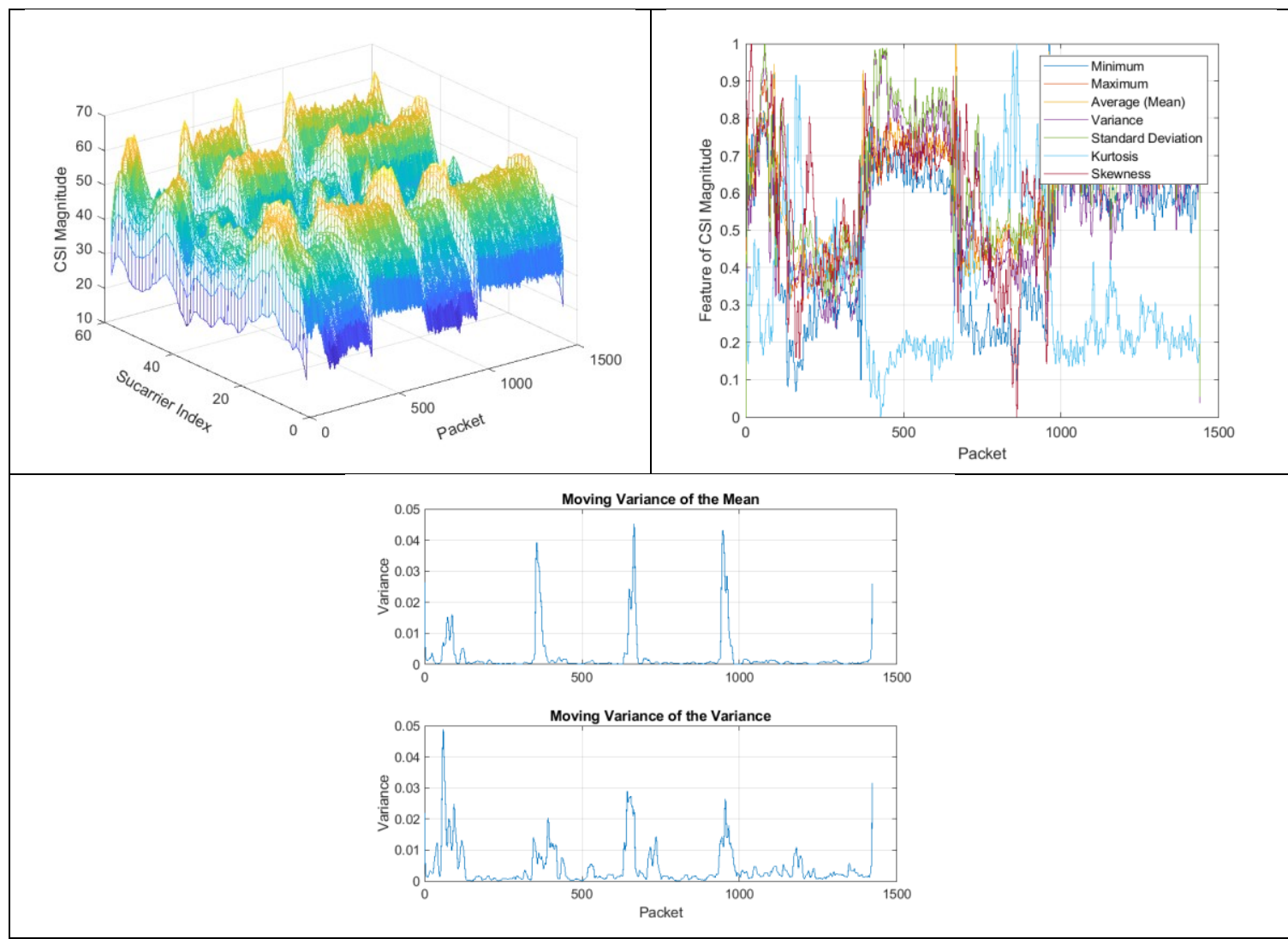

Figure 33 Sample of Plots for Data Collected by Subject 3 


\section{E.4 Subject $4(\mathrm{H})$}

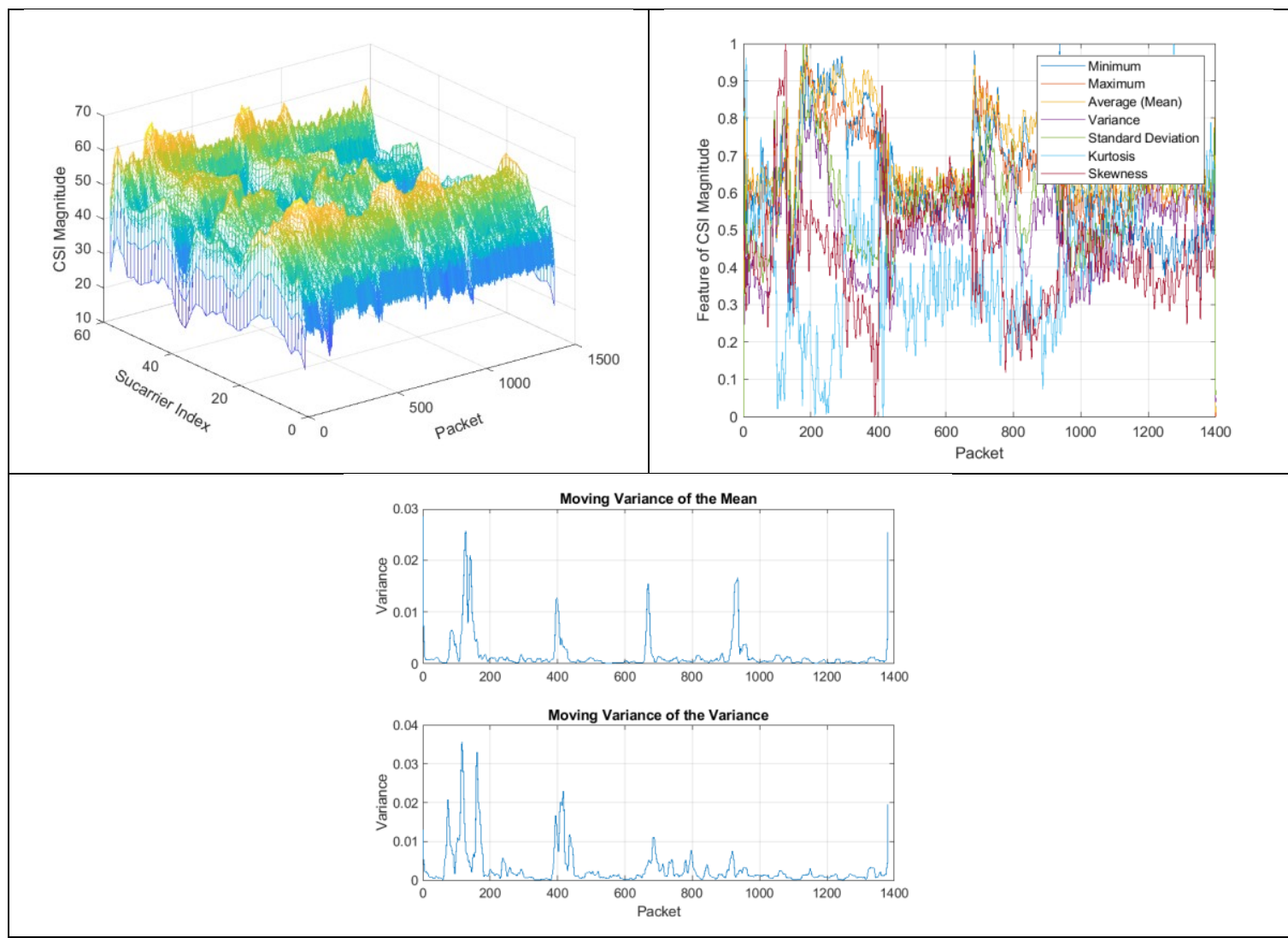

Figure 34 Sample of Plots for Data Collected by Subject 4 


\section{Bibliography or References}

[1] I. Joudeh et al, "WiFi Channel State Information-Based Recognition of SittingDown and Standing-Up Activities," in IEEE International Symposium on Medical Measurements and Applications (MeMeA), Istanbul, Turkey, 2019.

[2] C. Zhu and W. Sheng, "Realtime Recognition of Complex Human Daily Activities Using Human Motion and Location Data," in IEEE Transactions on Biomedical Engineering, vol. 59, (9), pp. 2422-2430, 2012.

[3] B. Wallace, R. Goubran, and F. Knoefel, “Cognition Assessment: A Framework for Episodic Measurement," in IEEE International Symposium on Medical Measurements and Applications (MeMeA), Benevento, Italy, pp. 1-6, 2016.

[4] B. Wallace et al, "Monitoring cognitive ability in patients with moderate dementia using a modified "whack-a-mole," in IEEE International Symposium on Medical Measurements and Applications (MeMeA), Rochester, MN, USA, 2017.

[5] B. Wallace et al, "Detecting Cognitive Ability Changes in Patients With Moderate Dementia Using a Modified "Whack-a-Mole" Game," in IEEE Transactions on Instrumentation and Measurement (TIM), vol. 67, (7), pp. 1521-1534, 2018. 
[6] K. K. Htike, O. O. Khalifa, H. A. M. Ramli, and M. A. M. Abushariah, "Human Activity Recognition for Video Surveillance using Sequences of Postures," in International Conference on e-Technologies and Networks for Development (ICeND2014), Beirut, Lebanon, 2014.

[7] M. K. Fiaz and B. Ijaz, "Vision Based Human Activity Tracking Using Artificial Neural Networks," in International Conference on Intelligent and Advanced Systems, Manila, Philippines, 2010.

[8] O. Bodilovskyi and A. Popov, "Estimation of Time Domain Parameters for Camera-Based Respiration Monitoring," in Signal Processing Symposium (SPSympo), Jachranka, Poland, 2017.

[9] R. Alfuadi and K. Mutijarsa, "Classification Method for Prediction of Human Activity Using Stereo Camera," in International Seminar on Application for Technology of Information and Communication (ISemantic), Semarang, Indonesia, 2016.

[10] X. Cai, G. Han, and J. Wang, "Assessment of Gait Parameters during Unconstrained Straight-line Walking using a Single Camera," in IEEE International Conference on Awareness Science and Technology (iCAST), Qinhuangdao, China, 2015. 
[11] K. Song and W. Chen, "Human Activity Recognition Using a Mobile Camera," in International Conference on Ubiquitous Robots and Ambient Intelligence (URAI), Incheon, South Korea, 2011.

[12] M. Z. Uddin, N. D. Thang, and T. Kim, "Human Activity Recognition via 3-D Joint Angle Features and Hidden Markov Models," in IEEE International Conference on Image Processing (ICIP), Hong Kong, China, 2010.

[13] M.W.C.N. Moladande and B.G.D.A. Madhusanka, "Implicit Intention and Activity Recognition of Human Using Neural Networks for a Service Robot Eye," in International Research Conference on Smart Computing and Systems Engineering (SCSE), Colombo, Sri Lanka, Sri Lanka, 2019.

[14] K. Avgerinakis, K. Adam, A. Briassouli, and Y. Kompatsiaris, "Moving Camera Human Activity Localization and Recognition with Motionplanes and Multiple Homographies," in IEEE International Conference on Image Processing (ICIP), Quebec City, QC, Canada, 2015.

[15] N. Käse, M. Babaee, and G. Rigoll, "Multi-view Human Activity Recognition using Motion Frequency," in IEEE International Conference on Image Processing (ICIP), Beijing, China, 2017. 
[16] B. Wallace, T. El Harake, R. Goubran, N. Valech, and F. Knoefel, "Preliminary Results for Measurement and Classification of Overnight Wandering by Dementia Patient using Multi-Sensors," in IEEE International Instrumentation Measurement Technology Conference (I2MTC), pp. 1-6, 2018.

[17] L. Russell, R. Goubran, and F. Kwamena, "Posture Sensing using a Low-cost Temperature Sensor Array," in IEEE International Symposium on Medical Measurements and Applications (MeMeA), Rochester, MN, USA, pp. 443-447, 2017.

[18] L. Russell, R. Goubran, and F. Kwamena, "Posture Detection Using Sounds and Temperature: LMS-Based Approach to Enable Sensory Substitution," in IEEE Transactions on Instrumentation and Measurement (TIM), vol. 67, (7), pp. 15431554, 2018.

[19] N. A. Capela, E. D. Lemaire, and N. Baddour, "Improving Classification of Sit, Stand, and Lie in a Smartphone Human Activity Recognition System," in Proceedings of IEEE International Symposium on Medical Measurements and Applications (MeMeA), Torino, Italy, pp. 473-478, 2015.

[20] G. De Leonardis et al, "Human Activity Recognition by Wearable Sensors: Comparison of Different Classifiers for Real-Time Applications," in IEEE 
International Symposium on Medical Measurements and Applications (MeMeA), Rome, Italy, pp. 1-6, 2018.

[21] A. Lopez, D. Perez, F. J. Ferrero, and O. Postolache, "A Real-Time Algorithm to Detect Falls in the Elderly," in IEEE International Symposium on Medical Measurements and Applications (MeMeA), Rome, Italy, pp. 1-5, 2018.

[22] B. Andò et al, "An event polarized paradigm for ADL detection in AAL context," in Proceedings of IEEE International Instrumentation and Measurement Technology Conference (I2MTC), Montevideo, Uruguay, 2014.

[23] B. Andò, S. Baglio, C. O. Lombardo, and V. Marletta, "An Event Polarized Paradigm for ADL Detection in AAL Context," in IEEE Transactions on Instrumentation and Measurement (TIM), vol. 64, (7), pp. 1814-1825, 2015.

[24] B. Andò, S. Baglio, C. O. Lombardo, and V. Marletta, "A Multisensor Data-Fusion Approach for ADL and Fall Classification," in IEEE Transactions on Instrumentation and Measurement (TIM), vol. 65, (9), pp. 1960-1967, 2016.

[25] Z. Wang et al, "Inertial Sensor-Based Analysis of Equestrian Sports Between Beginner and Professional Riders Under Different Horse Gaits," in IEEE Transactions on Instrumentation and Measurement (TIM), vol. 67, (11), pp. 26922704, 2018. 
[26] A. Yurtman and B. Barshan, "Novel Non-Iterative Orientation Estimation for Wearable Motion Sensor Units Acquiring Accelerometer, Gyroscope, and Magnetometer Measurements," in IEEE Transactions on Instrumentation and Measurement (TIM) (Early Access), pp. 1-1, 2019.

[27] S. Matsuguma and A. Kajiwara, "Bathroom accident detection with 79GHz-band millimeter wave sensor," in IEEE Sensors Applications Symposium (SAS), Sophia Antipolis, France, 2019.

[28] T. L. M. van Kasteren, G. Englebienne, and B. J. A. Kröse, “An activity monitoring system for elderly care using generative and discriminative models," in Personal and Ubiquitous Computing, vol. 14, (6), pp 489-498, 2010.

[29] Z. Gu et al, "Blind Separation of Doppler Human Gesture Signals Based on Continuous-Wave Radar Sensors," in IEEE Transactions on Instrumentation and Measurement (TIM), vol. 68, (7), pp. 2659-2661, 2019.

[30] S. K. Leem, F. Khan, and S. H. Cho, "Detecting Mid-air Gestures for Digit Writing with Radio Sensors and a CNN," in IEEE Transactions on Instrumentation and Measurement (TIM) (Early Access), pp. 1-1, 2019. 
[31] Z. Wang, B. Guo, Z. Yu, and X. Zhou, "Wi-Fi CSI-Based Behavior Recognition: From Signals and Actions to Activities," in IEEE Communications Magazine, vol. 56, (5), pp. 109-115, 2018.

[32] Y. Zeng, P. Pathak, and P. Mohapatra, "WiWho: Wifi-based Person Identification in Smart Spaces," in Proceedings of the 15th International Conference on Information Processing in Sensor Networks, pp. 1-12, 2016.

[33] N. Ghourchian, M. Allegue-Martinez, and D. Precup, "Real-Time Indoor Localization in Smart Homes using Semi-Supervised Learning," in Proceedings of the 29th AAAI Conference on Innovative Applications (IAAI-17), pp. 4670-4677, 2017.

[34] D. Alshamaa, A. Chkeir, F. Mourad-Chehade, and P. Honeine, “A Hidden Markov Model for Indoor Trajectory Tracking of Elderly People," in IEEE Sensors Applications Symposium (SAS), Sophia Antipolis, France, 2019.

[35] Q. Gao, J. Wang, X. Ma, X. Feng, and H. Wang, “CSI-Based Device-Free Wireless Localization and Activity Recognition Using Radio Image Features," in IEEE Transactions on Vehicular Technology, vol. 66, (11), pp. 10346-10356, 2017. 
[36] M. Miyazaki, S. Ishida, A. Fukuda, T. Murakami, and S. Otsuki, "Initial Attempt on Outdoor Human Detection using IEEE802.11ac WLAN Signal,” in IEEE Sensors Applications Symposium (SAS), Sophia Antipolis, France, 2019.

[37] J. Liu et al, "Tracking Vital Signs During Sleep Leveraging Off-the-shelf WiFi," in Proceedings of the 16th ACM International Symposium on Mobile Ad Hoc Networking and Computing, pp. 267-276, 2015.

[38] X. Wang, C. Yang, and S. Mao, "PhaseBeat: Exploiting CSI Phase Data for Vital Sign Monitoring with Commodity WiFi Devices," in IEEE 37th International Conference on Distributed Computing Systems (ICDCS), pp. 1230-1239, 2017.

[39] N. Patwari, L. Brewer, Q. Tate, O. Kaltiokallio, and M. Bocca, "Breathfinding: A Wireless Network That Monitors and Locates Breathing in a Home," in IEEE Selected Topics in Signal Processing, vol. 8, (1), pp. 30-42, 2014.

[40] L. Wang, K. Sun, H. Dai, A. X. Liu, and X. Wang, "WiTrace: Centimeter-level passive gesture tracking using WiFi signals," in IEEE 15th Annual International Conference on Sensing, Communication, and Networking (SECON), pp. 1-9, 2018.

[41] K. Ali, A. X. Liu, W. Wang, and M. Shahzad, "Keystroke Recognition Using Wifi Signals," in Proceedings of the 21st Annual ACM International Conference on Mobile Computing and Networking, pp. 90-102, 2015. 
[42] Y. Wang et al, "E-eyes: Device-free Location-oriented Activity Identification using Fine-grained WiFi Signatures," in Proceedings of the 20th Annual International Conference on Mobile Computing and Networking, pp. 617-628, 2014.

[43] W. Wang, A. X. Liu, M. Shahzad, K. Ling, and S. Lu, "Understanding and Modeling of WiFi Signal based Human Activity Recognition," in Proceedings of the 21st Annual International Conference on Mobile Computing and Networking, pp. 65-76, 2015.

[44] H. Li, K. Ota, M. Dong, and M. Guo, "Learning Human Activities through Wi-Fi Channel State Information with Multiple Access Points," in IEEE Communications Magazine, vol. 56, (5), pp. 124-129, 2018.

[45] B. Tan et al, "Exploiting WiFi Channel State Information for Residential Healthcare Informatics," in IEEE Communications Magazine, vol. 56, (5), pp. 130137, 2018.

[46] W. Li, B. Tan, and R. J. Piechocki, "WiFi-based Passive Sensing System for Human Presence and Activity Event Classification," in IET Wireless Sensor Systems, vol. 8, (6), pp. 276-283, 2018. 
[47] Z. Chen, L. Zhang, C. Jiang, Z. Cao, and W. Cui, "WiFi CSI Based Passive Human Activity Recognition Using Attention Based BLSTM," in IEEE Transactions on Mobile Computing, pp. 1-12, 2018.

[48] E. Alpaydin, Introduction to Machine Learning. Cambridge, MA: Massachusetts Institute of Technology (MIT) Press, 2010.

[49] M. I. Jordan and T. M. Mitchell, "Machine learning: Trends, perspectives, and prospects," in Science, vol. 349, (6245), pp. 255-260, 2015.

[50] Y. LeCun, Y. Bengio, and G. Hinton, "Deep learning," in Nature, vol. 521, (7553), pp. 436-444, 2015.

[51] Aerial Technologies, “Aerial Solution Setup Guide,” Montreal, QC, 2018.

[52] H. He, Y. Bai, E. A. Garcia, and S. Li, "ADASYN: Adaptive Synthetic Sampling Approach for Imbalanced Learning," in Proceedings of IEEE International Joint Conference on Neural Networks, Hong Kong, China, pp. 1322-1328, 2018. 